\title{
THE NANOGRAV NINE-YEAR DATA SET: OBSERVATIONS, ARRIVAL TIME MEASUREMENTS, AND ANALYSIS OF 37 MILLISECOND PULSARS
}

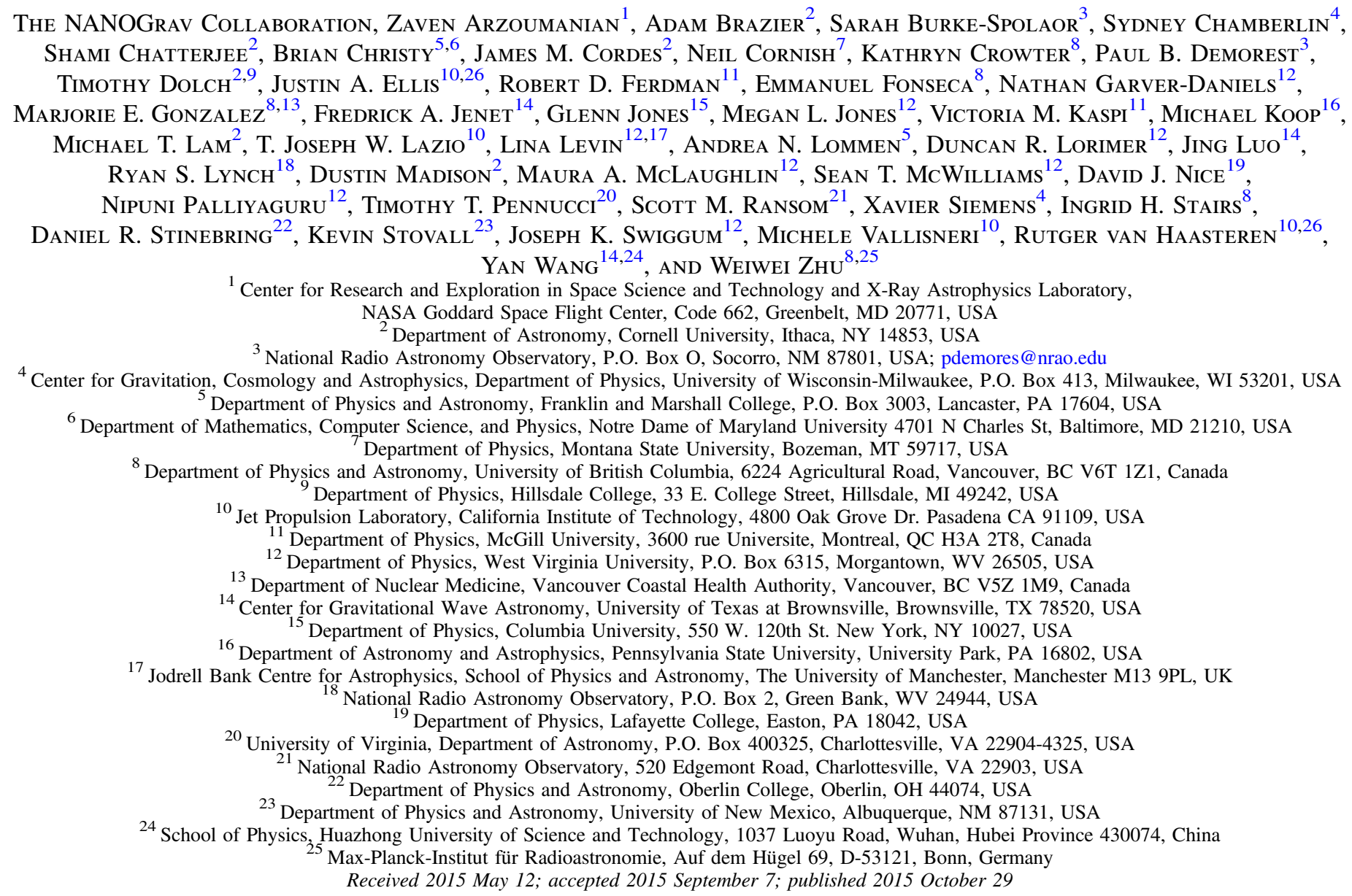

\section{ABSTRACT}

We present high-precision timing observations spanning up to nine years for 37 millisecond pulsars monitored with the Green Bank and Arecibo radio telescopes as part of the North American Nanohertz Observatory for Gravitational Waves (NANOGrav) project. We describe the observational and instrumental setups used to collect the data, and methodology applied for calculating pulse times of arrival; these include novel methods for measuring instrumental offsets and characterizing low signal-to-noise ratio timing results. The time of arrival data are fit to a physical timing model for each source, including terms that characterize time-variable dispersion measure and frequency-dependent pulse shape evolution. In conjunction with the timing model fit, we have performed a Bayesian analysis of a parameterized timing noise model for each source, and detect evidence for excess lowfrequency, or "red," timing noise in 10 of the pulsars. For 5 of these cases this is likely due to interstellar medium propagation effects rather than intrisic spin variations. Subsequent papers in this series will present further analysis of this data set aimed at detecting or limiting the presence of nanohertz-frequency gravitational wave signals.

Key words: gravitational waves - methods: data analysis - pulsars: general

\section{INTRODUCTION}

The era of gravitational-wave astronomy is expected to begin within the next decade. It will be heralded by the first direct detection of gravitational waves as perturbations in the spacetime metric due to acceleration of massive objects.

\footnotetext{
${ }^{26}$ Einstein Fellow.
}

Anticipated gravitational wave sources include merging systems of supermassive black hole binaries (SMBHBs) and neutron-star binaries, as well as inflation-era relics (e.g., Grishchuk 2005) and cosmic strings (e.g., Vilenkin \& Shellard 1994). Several major experiments are underway in order to detect and characterize gravitational waves. One type of experiment is a pulsar timing array (PTA), in which a collection of radio pulsars is monitored, providing sensitivity to 
gravitational radiation in the nanohertz region of the spectrum (Hobbs et al. 2010).

Millisecond pulsar (MSP) rotation is very stable, and pulsars in relativistic binary systems have already been used to place the most stringent experimental constraints on strong-field gravity so far. The orbital decay observed in such binary systems serves as compelling indirect evidence of gravitational radiation, as the observed orbital decay rates match the expected rates due to loss of energy and angular momentum via emission of gravitational waves (Kramer et al. 2006; Weisberg et al. 2010; Fonseca et al. 2014). Sazhin (1978) and Detweiler (1979) were the first to suggest that pulsar signals can be used to directly measure gravitational waves, particularly those produced by SMBHB mergers. Hellings \& Downs (1983) extended this view and developed the notion that gravitational waves produce pulse time-of-arrival (TOA) shifts that are correlated among a set of pulsars. In principle, this allows the gravitational wave signal to be unambiguously separated from other astrophysical phenomena affecting measured TOAs - these would be specific to each pulsar, thus uncorrelated between different objects.

The North American Nanohertz Observatory for Gravitational Waves (NANOGrav) ${ }^{27}$ is one of several PTA programs across the globe that collectively form the International Pulsar Timing Array (IPTA; Hobbs et al. 2010). These collaborations regularly monitor the most stable members of the MSP population distributed across the sky in order to achieve the highest sensitivity possible toward gravitational wave detection. While no direct detection has been made so far, individual PTA programs have yielded upper limits on the amplitude $A_{\mathrm{gw}}$ of the characteristic gravitational-wave strain $h_{\mathrm{c}}$ due to a stochastic background in the nanohertz regime (van Haasteren et al. 2011; Demorest et al. 2013; Shannon et al. 2013; Lentati et al. 2015), where

$$
h_{\mathrm{c}}(f)=A_{\mathrm{gw}}\left(\frac{f}{1 \mathrm{yr}^{-1}}\right)^{\alpha}
$$

and $f$ is the observed gravitational wave frequency. A number of predictions for the expected strength of the SMBHB gravitational wave background have been made (e.g., Jaffe \& Backer 2003; Sesana et al. 2008; Sesana 2013; McWilliams et al. 2014), with $\alpha=-2 / 3$ and expected values for $A_{\mathrm{gw}}$ ranging from $\sim 5 \times 10^{-16}$ to $4 \times 10^{-15}$. Currently, the best published experimental limit is $A_{\mathrm{gw}}<2.7 \times 10^{-15}$ (Shannon et al. 2013). In addition to measuring the gravitational wave background, PTA measurements can be used to attempt to measure other gravitational wave signals, such as periodic gravitational waves from individual sources (Sesana et al. 2009; Arzoumanian et al. 2014) and permanent deformations in spacetime, referred to as gravitational-wave "memory" (e.g., Madison et al. 2014; Wang et al. 2015). PTA data can also be used for ancillary studies of pulsars, binary systems, and the interstellar medium (ISM).

In this study, we extend the data set analyzed by Demorest et al. (2013) and present high-precision timing observations of the updated NANOGrav PTA. The data comprise measurements of 37 MSPs and span nine years of observation. In Section 2, we provide information regarding the telescopes, methods and pulsar backends used for data collection. In

\footnotetext{
27 http://www.nanograv.org
}

Section 3, we describe the general procedure for data reduction, flux and polarization calibration, TOA determination and data excision. In Section 4, we outline the strategy used for generating updated timing models for each pulsar and discuss the results of the model fitting. In Section 5, we describe the models used for characterizing noise in our timing data. In Section 6, we summarize the results and implications of this work. Raw and processed data products presented here are publicly available as of the date this work is published (Section 4).

\section{OBSERVATIONS}

This paper reports on observations of an array of MSPs made over a 9-year span from 2004 to 2013. Pulsars were chosen for this project based on expectations of high TOA precision, reliable detection across a wide range of frequencies, and lack of unpredictable timing fluctuations from astrophysical effects (for example, no eclipsing binary pulsars have been included). The array initially included 15 pulsars, and it grew to 37 pulsars over the course of the project. The growth came from the discovery of new MSPs and the advent of wide-band data acquisition systems, which allowed observation of some sources previously deemed too weak or unreliable. The first five years of data on 17 of the pulsars were previously reported by Demorest et al. (2013), however all data have been reprocessed as described in following sections.

Pulsars with declinations in the range $0^{\circ}<\delta<39^{\circ}$ were observed with the $305 \mathrm{~m}$ William E. Gordon Telescope of the Arecibo Observatory. Sources outside this declination range were observed with the $100 \mathrm{~m}$ Robert C. Byrd Green Bank Telescope (GBT) of the National Radio Astronomy Observatory. Two sources were observed at both telescopes, PSRs $\mathrm{J} 1713+0747$ and $\mathrm{B} 1937+21$.

Table 1 summarizes the radio frequencies and data acquisition systems used for this project; these are discussed in more detail below. Observation time spans for individual sources are listed in Table 2, and observation dates are displayed in Figure 1.

Sources were observed at approximately monthly intervals through most of this program, with denser observations, every three weeks, in 2013. Scheduling of individual observing epochs varied depending on telescope operational considerations and sometimes deviated from regular cadences. Observations at both telescopes were interrupted in 2007 due to telescope painting (Arecibo) and azimuth track refurbishment (GBT).

Each pulsar was observed using radio receivers at two separate frequencies throughout this program in order to measure and remove frequency-dependent (FD) dispersive effects. Exceptions were Arecibo observations of five sources that were made at a single frequency before 2009 or 2011 (depending on the source), and certain Arecibo observations during 2012, when technical issues impeded use of the $430 \mathrm{MHz}$ receiver.

At Arecibo, observations of a given pulsar using two receivers were made in immediate succession within $\sim 1 \mathrm{hr}$. At the GBT, observations using two receivers were typically separated by a few days due to the need for a physical receiver change at that telescope. Observations without complementary data from the other receiver taken within 14 days were excluded from the data set. Exceptions to this rule were made for early Arecibo single-receiver observations of several 
Table 1

Observing Frequencies and Bandwidths

\begin{tabular}{|c|c|c|c|c|c|c|}
\hline \multirow[b]{2}{*}{$\begin{array}{l}\text { Telescope } \\
\text { Receiver }\end{array}$} & \multicolumn{3}{|c|}{ ASP/GASP } & \multicolumn{3}{|c|}{ PUPPI/GUPPI } \\
\hline & Data $\operatorname{Span}^{\mathrm{a}}$ & $\begin{array}{c}\text { Frequency } \\
\text { Range }^{\mathrm{b}} \\
(\mathrm{MHz})\end{array}$ & $\begin{array}{c}\text { Usable } \\
\text { Bandwidth }^{\mathrm{c}} \\
(\mathrm{MHz})\end{array}$ & Data $\operatorname{Span}^{\mathrm{a}}$ & $\begin{array}{c}\text { Frequency } \\
\text { Range }^{\mathrm{b}} \\
(\mathrm{MHz})\end{array}$ & $\begin{array}{c}\text { Usable } \\
\text { Bandwidth }^{\mathrm{c}} \\
(\mathrm{MHz})\end{array}$ \\
\hline \multicolumn{7}{|c|}{ Arecibo } \\
\hline 327 & $2005.0-2012.0$ & 315-339 & 34 & 2012.2-2013.8 & $302-352$ & 50 \\
\hline 430 & 2005.0-2012.3 & $422-442$ & 20 & 2012.2-2013.8 & $421-445$ & 24 \\
\hline L-wide & 2004.9-2012.3 & $1380-1444$ & 64 & 2012.2-2013.8 & $1147-1765$ & 603 \\
\hline S-wide & 2004.9-2012.6 & $2316-2380$ & 64 & $2012.2-2013.8$ & $1700-2404^{\mathrm{d}}$ & 460 \\
\hline \multicolumn{7}{|c|}{ GBT } \\
\hline Rcvr_800 & $2004.6-2011.0$ & $822-866$ & 64 & 2010.2-2013.8 & $722-919$ & 186 \\
\hline Rcvr1_2 & $2004.6-2010.8$ & $1386-1434$ & 48 & 2010.2-2013.8 & 1151-1885 & 642 \\
\hline
\end{tabular}

Notes.

a Dates of instrument use. Observation dates of individual pulsars vary; see Figure 1.

b Most common values; some observations differed. Some frequencies unusable due to radio frequency interference.

${ }^{c}$ Nominal values after excluding narrow subbands with radio frequency interference.

d Non-contiguous usable bands at 1700-1880 and 2050-2404 MHz.

sources mentioned above, and for wide-band $1400 \mathrm{MHz}$ data, in which the wide frequency range of one receiver partially made up for the lack of data from a second receiver. The typical observation duration was about 25 minutes, with some variations over the course of the program.

All receivers are sensitive to dual linear polarizations, with the exception of the Arecibo $430 \mathrm{MHz}$ receiver, which measures dual circular polarizations. Polarization cross-products were recorded so that full Stokes parameters could be recovered. However, for the present work, we only use totalintensity measurements, obtained by summing the calibrated signals from pairs of orthogonal polarizations.

Two sets of data acquisition systems were used. Early observations (through 2012.3 at Arecibo and through 2011.0 at Green Bank) were recorded by the nearly identical Astronomical Signal Processor (ASP) and Green Bank Astronomical Signal Processor (GASP) data acquisition systems (Demorest 2007). Later observations (beginning 2012.2 at Arecibo and 2010.2 at Green Bank) were recorded using the nearly identical Puerto Rican Ultimate Pulsar Processing Instrument (PUPPI) and the Green Bank Ultimate Pulsar Processing Instrument (GUPPI; DuPlain et al. 2008; Ford et al. 2010). Each of these systems digitized incoming baseband radio telescope voltage signals at the appropriate Nyquist rate, channelized them into subbands, performed real-time coherent dedispersion, calculated self- and cross-products to record full polarization information, and folded the data with the dynamically calculated pulsar period using a pre-computed ephemeris. The end product of each instrument was folded pulse profiles (2048 bins) with self- and cross-products recorded over a series of frequency channels and integrated over short subintervals over the course of an observation.

The ASP and GASP systems processed up to $64 \mathrm{MHz}$ of bandwidth, recording in contiguous $4 \mathrm{MHz}$ subbands. Data were usually recorded in consecutive 60-s subintervals over the course of an observation.

The PUPPI and GUPPI systems processed 100, 200, or $800 \mathrm{MHz}$ of bandwidth, depending on the mode of operation. In each case, data were recorded in contiguous subbands of width $1.5625 \mathrm{MHz}$. Data were usually recorded in consecutive 10-s subintervals, with 1-s subintervals used for some Arecibo $1400 \mathrm{MHz}$ observations to aid interference excision.

In some cases, particularly with PUPPI and GUPPI, bandwidth was limited by telescope receivers rather than the data acquisition instrument (Table 1). In post-processing, narrowband radio frequency noise was removed and adjacent subbands were summed before arrival times were calculated (see Section 3.1).

Each pulsar observation was preceded or followed by measurement of a pulsed noise signal using a setup identical to the pulsar observation in order to calibrate the signal levels. The pulsed noise signals themselves were calibrated in on- and off-source observations of unpolarized continuum radio sources on a monthly basis.

For the timing analysis in this paper, only the polarization self-products were used. Data were summed in time and polarization (Section 3.1). Simultaneous observations between ASP and PUPPI at Arecibo, and between GASP and GUPPI at the GBT, were used to measure the time offset between these pairs of instruments (Appendix A).

\section{CALIBRATION AND TOA DETERMINATION}

The results of the observations described in Section 2 are "raw" pulse profiles. This section describes the procedures employed to turn the raw profiles into usable pulse TOAs. These included: RFI excision, polarization and flux calibration, additional averaging in time and frequency, derivation of template profiles, and finally TOA determination. All data processing operations described in this section were carried out using the PSRCHIVE software package (Hotan et al. 2004; van Straten et al. 2012). ${ }^{28}$ These were organized into a processing pipeline via a set of scripts that are available online. ${ }^{29}$ Overall, the calibration and processing strategy used here is similar to Demorest et al. (2013), and is based on standard methods for pulsar data analysis.

\footnotetext{
${ }^{28}$ http://psrchive.sourceforge.net; PSRCHIVE version 2015-01-15 b4826eb was used for this work.

${ }^{29}$ http://github.com/demorest/nanopipe
} 
Table 2

Basic Pulsar Parameters and TOA Statistics

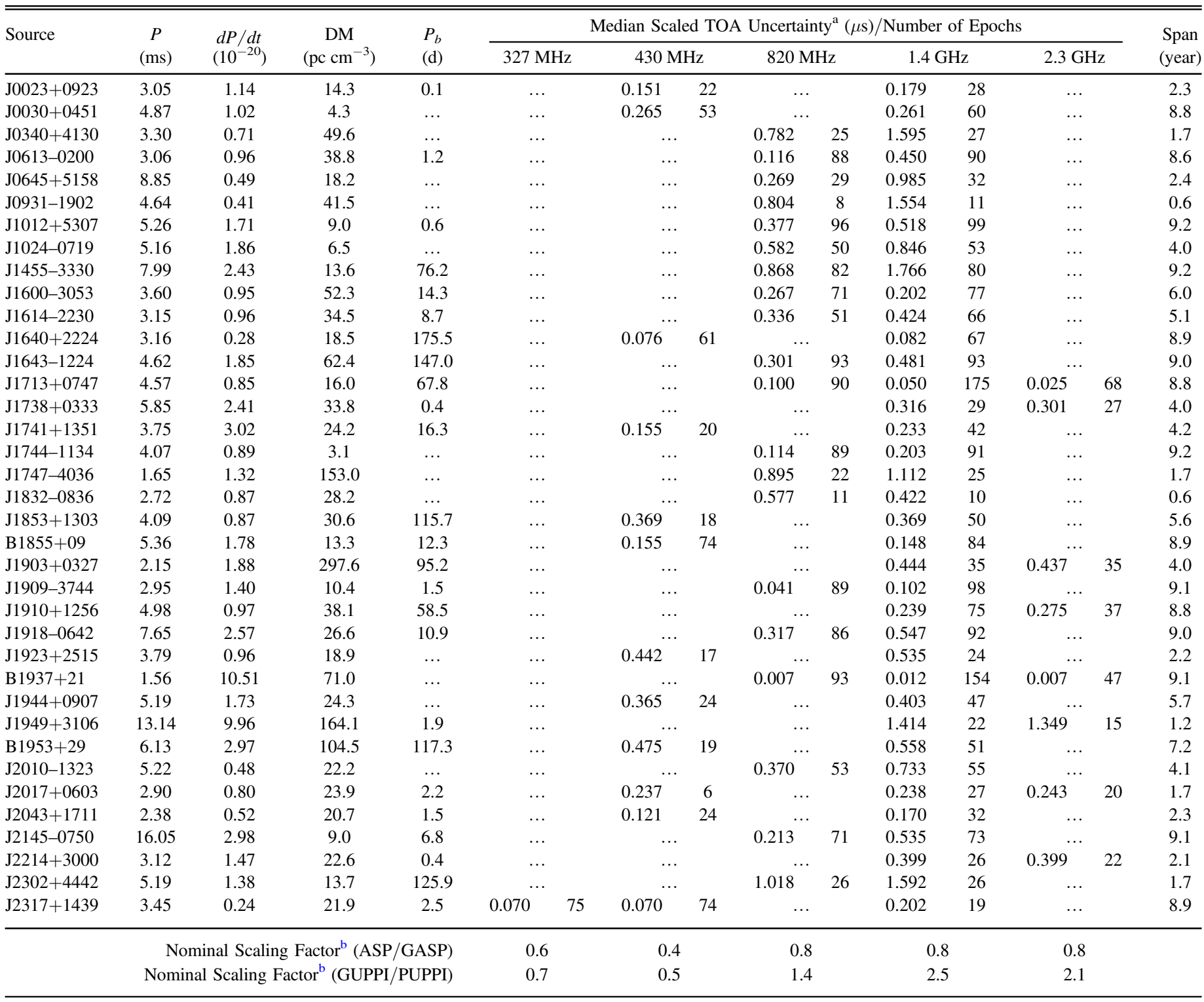

Notes. ${ }^{a}$ For this table, the original TOA uncertainties were scaled by their bandwidth-time product $\left(\frac{\Delta \nu}{100 \mathrm{MHz}} \frac{\tau}{1800 \mathrm{~s}}\right)^{1 / 2}$ to remove variation due to different instrument
bandwidths and integration time.

${ }^{b}$ TOA uncertainties can be rescaled to the nominal full instrumental bandwidth as listed in Table 1 by dividing by the scaling factors given here.

\subsection{Calibration and Averaging}

The polarization calibration procedure used for these data sets was identical to that described by Demorest et al. (2013): a locally generated broadband noise source is pulsed at $25 \mathrm{~Hz}$, split into two copies, and coupled in to the two polarization signal paths. Before each pulsar observation, a short $(\sim 1$ minute) observation of the pulsed noise signal is recorded by the backend systems. This correlated noise source observation is used to calibrate the two leading polarization terms: differential gain and phase between the two hands of polarization. The noise source power is not assumed to be equal in each hand-its power in each polarization is measured separately at each observing epoch by observing the noise source while the telescope is pointed on and off a bright, unpolarized quasar (B1442+101 at Green Bank; J1413+1509 at Arecibo). For purposes of this paper, we used this calibration to balance the gains of orthogonal polarizations before summing to produce total intensity profiles used for pulse timing. While we have not solved for a complete polarization calibration solution here, the calibration data can also be used to create full-polarimetry profiles and to flux-calibrate the pulse profiles using the known flux densities of the quasars used as calibration sources.

Following calibration, excision of data corrupted by radio frequency interference (RFI) was performed in two steps. First, a set of consistently bad frequency ranges for each telescope receiver was determined manually and was removed from all 


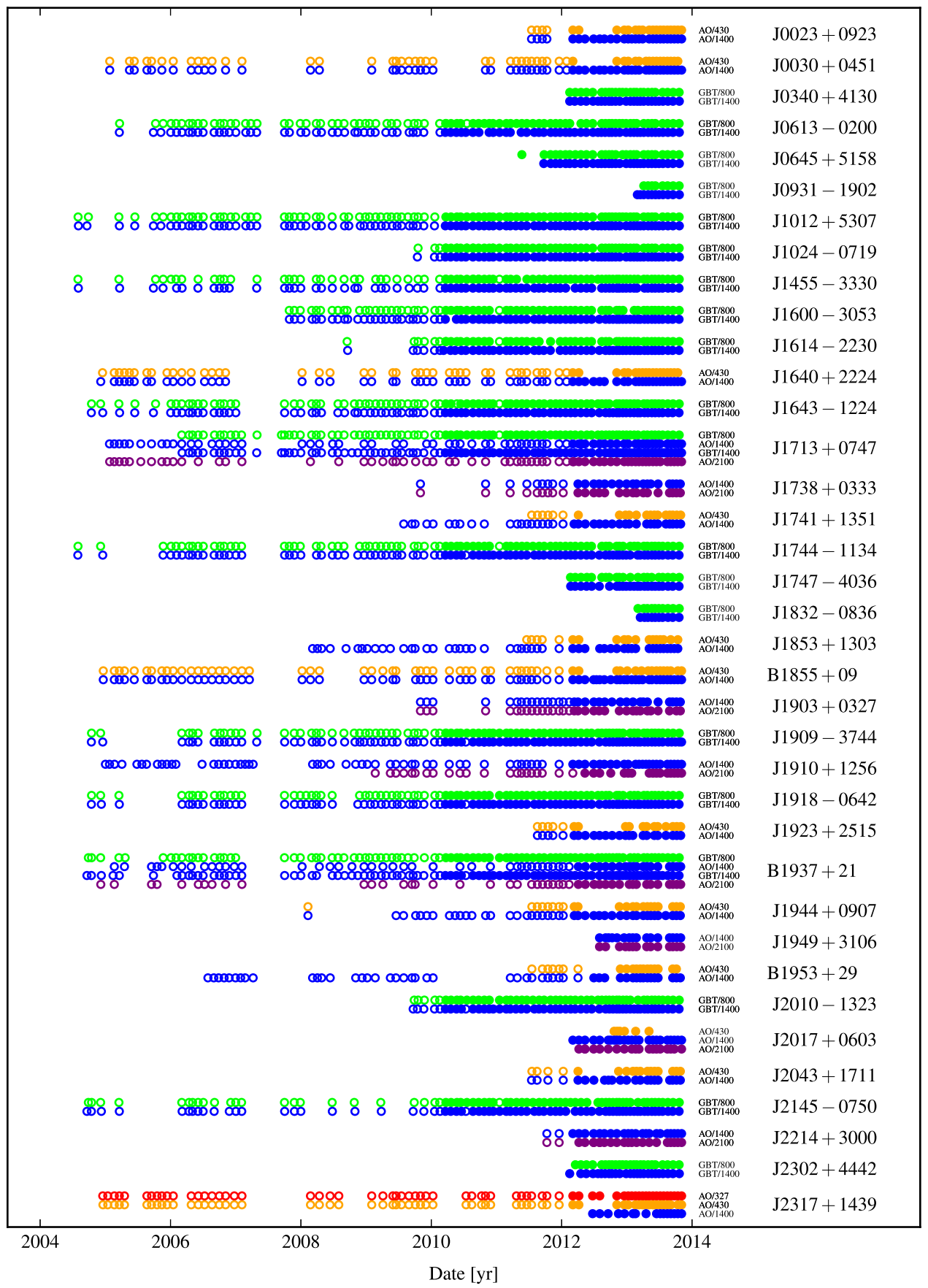

Figure 1. Epochs of all observations in the data set. Marker type indicates data acquisition system: open circles are ASP or GASP; closed circles are PUPPI or GUPPI. Colors indicate radio frequency band, at either telescope: red is $327 \mathrm{MHz}$; orange is $430 \mathrm{MHz}$; green is $820 \mathrm{MHz}$; blue is $1.4 \mathrm{GHz}$; and purple is $2.1 \mathrm{GHz}$.

data sets. The interfering signals primarily responsible for these cuts are satellite transmissions near $\sim 1.6 \mathrm{GHz}$ and radar signals near $\sim 1.2 \mathrm{GHz}$. This step resulted in removal of up to $15 \%$ of the full bandwidth recorded by GUPPI and PUPPI; the narrowband ASP and GASP instruments were tuned to avoid these strong signals. The remaining usable bandwidth for each receiver and data acquisition system is listed in Table 1. Following this initial cut, remaining RFI was removed via the median filter algorithm in PSRCHIVE. In each 20-channel wide frequency window, the median off-pulse variation was computed, and any channels exceeding four times this value were removed. Finally, prior to the final averaging described below, all profiles were normalized to have constant off-pulse variance. This step acted to down-weight any remaining corrupted data.

As the TOA-determination procedure described in the next section begins to fail at very low signal-to-noise ratios $(\mathrm{S} / \mathrm{N})$ (see Appendix B), it is advantageous to average as much data as possible into each profile before measuring a TOA. The final time and frequency resolution that should be used is ultimately limited by the need to resolve TOA shifts as a function of time (for example, from orbital motion) or frequency (from profile frequency evolution or variable dispersion measure; DM). We chose to average profiles in time up to a maximum of 30 minutes or $2 \%$ of the pulsar's binary period, whichever is shorter. Data from each observing session were divided equally 
-for example, a 40-minute observation would be averaged into two 20-minute sections. The shortest averaging was for PSR J0023+0923, which has an orbital period of 200 minutes (see $P_{\mathrm{b}}$ column in Table 2). For frequency averaging, we adopted a slightly different strategy for each instrument. For ASP and GASP, no frequency averaging was done, and the final profile data remain at the instrumental resolution of $4 \mathrm{MHz}$. For GUPPI and PUPPI, the data were averaged to different final frequency resolutions depending on which receiver system was in use: $1.5625 \mathrm{MHz}$ for $327 \mathrm{MHz}$ and $430 \mathrm{MHz}$ data, $3.125 \mathrm{MHz}$ for $0.82 \mathrm{GHz}$, and $12.5 \mathrm{MHz}$ for all frequencies above $1 \mathrm{GHz}$.

To summarize, the profile data set for any given observation consists of calibrated total-intensity profiles collected simultaneously across many subbands (typically between 5 and 60) divided into one or more subintervals (typically 20-30 minutes).

\subsection{Measuring Times of Arrival}

We calculated a pulse TOA from each averaged profile resulting from the procedure described in the previous section. Thus any given observation results in a large number of TOAs, computed from data collected simultaneously in different subbands.

We calculated TOAs using the Fourier-domain algorithm of Taylor (1992) as implemented in the PSRCHIVE program pat. This method determined each TOA and its uncertainty via a least-squares fit for the pulse phase shift between an observed total-intensity pulse profile and an ideal template profile. Template determination was done using the same procedure as Demorest et al. (2013). In short, for each pulsar and each receiver, we made a signal-to-noise-weighted sum of all GUPPI/PUPPI profile data. We then de-noised these profiles via wavelet decomposition and thresholding of the wavelet coefficients (as implemented in the PSRCHIVE program psrsmooth). The same template profiles were used to calculate TOAs from both GUPPI/PUPPI data and from GASP/ASP data. All templates were aligned so that phase zero occurs at the peak of the pulse profile.

The pairs of data acquisition systems used at each telescope (GASP and GUPPI or ASP and PUPPI) had different signal path lengths and different internal latencies, which led to systematic TOA offsets. These must be measured and removed in order to avoid corrupting the pulsar timing results. This has typically been done in the past using the pulsar TOA measurements themselves - a time offset between two systems is fit for, either as a term in the overall timing model fit (see Section 4), or separately using a subset of contemporaneous TOAs (e.g., Taylor \& Weisberg 1989). More recently, Manchester et al. (2013) applied a method where a locally generated timing signal was injected, measured and used to derive per-backend timing offsets. For the present work, we developed a new method that analyzes the noise in the pulsar profiles collected simultaneously with a given pair of data acquisition systems. In simultaneous data the noise is correlated, and cross-correlating the pulse profile data from the two systems provided much higher-precision offset measurements than could be made from TOAs, where by design most of the noise was filtered out by the templatematching process. The results were offsets between GASP and GUPPI (also ASP and PUPPI), with typical value $\sim 1 \mu$ s and uncertainty $\sim 5 \mathrm{~ns}$, that were applied directly to the TOAs. Additional details are presented in Appendix A.

\subsection{Editing TOA Data Sets}

After all TOAs were generated as described above, several cuts were made on the set of TOAs from each pulsar in order to arrive at the final set of data used in the analysis described in the next section.

1. For observations where simultaneous data were recorded with both sets of instrumentation, the ASP/GASP TOAs were removed.

2. In order to meaningfully determine a time-variable DM, data from observing epochs with low fractional bandwidth, $\nu_{\text {high }} / \nu_{\text {low }}<1.1$, were removed. In practice, this criterion removed TOAs for any pulsar that did not have, within any given 14-day window, either TOAs measured using two separate receivers or TOAs measured using one wide-band receiver with a wide-band data acquisition system.

3. As TOA measurement uncertainties become both underestimated and significantly non-Gaussian at low $\mathrm{S} / \mathrm{N}$, TOAs from profiles with an $\mathrm{S} / \mathrm{N}$ less than 8 were removed. See Appendix B for further analysis of this cut.

4. A small number of outlier TOAs were manually identified and removed during the timing analysis. Typically these were due to low-S/N data that were just above the $\mathrm{S} / \mathrm{N}$ cutoff or to RFI not excised by the algorithms described above. The TOAs removed by this process comprise $\sim 1 \%$ of the full data set.

All TOA data presented in this paper are publicly available. $^{30}$ TOAs removed from the analysis as described above are included as supplementary files along with the main data set. The TOAs are given in TEMPO2 format, with additional flags specifying relevant meta-data (e.g., backend, receiver, profile template file, etc.). This data format can be read using both TEMPO ${ }^{31}$ and TEMPO $2^{32}$ pulsar timing analysis software. Clock correction data needed to reference the TOAs to the TT (BIPM) timescale (see Section 4.1) are distributed along with the TOA data, in the standard formats used by TEMPO and TEMPO2.

\section{TIMING ANALYSIS}

We fit the TOA set for each pulsar to a timing model using standard procedures as described by Lorimer \& Kramer (2005), supplemented by novel methods to compensate for FD pulse shape variations and to model noise in the TOA data sets. In this section, we start by summarizing our use of standard timing models, and then we describe the new algorithm for handling FD pulse shape variations. In the next section of the paper, we describe the noise model.

\subsection{Timing Models}

All TOAs were initially measured using a local, topocentric time provided by hydrogen-maser clocks located at the

\footnotetext{
30 http://data.nanograv.org

31 http://tempo.sourceforge.net; TEMPO version 2014-11-20 76b8375 was used for this work.

${ }^{32}$ http://tempo2.sourceforge.net; TEMPO2 version 2014.11.1 was used for this work.
} 
observatories. Observatory clock corrections, determined by daily monitoring of the maser offsets compared to times determined using Global Positioning System (GPS) receivers, were used to transform the TOAs to Coordinated Universal Time (UTC). The times were further transformed to Terrestrial Time (TT) as published by the Bureau International des Poids et Mesures, TT(BIPM), after accounting for the effects of the Earth's varying rotation rate as published by the International Earth Rotation and Reference Systems Service (IERS). Finally, relativistic corrections are applied to convert the times to Barycentric Dynamical Time (TDB). ${ }^{33}$ Propagation delays in the solar system, used to project the TOAs to the Solar-System Barycenter (SSB) are calculated using the JPL DE421 planetary ephemeris, ${ }^{34}$ rotated into an ecliptic reference frame using the 2010 IAU value of the obliquity of the ecliptic.

We derived timing models by enumerating all rotations of each pulsar and accounting for the various physical processes, discussed below, that can cause observed timing delays. This modeling was done in conjunction with a parameterized model for noise in the arrival-time data, described in detail in Section 5 and Appendix C. In effect, the noise model determined a separate "weight" for each data subset, defined by the combination of receiver and backend system used, along with a measurement of temporally correlated "red" noise. We used the TEMPO and TEMPO2 pulsar-timing analysis programs, making use of recently implemented generalized least-squares (GLS) fitting procedures which take into account correlations in the TOA noise when determining timing model parameter values and their uncertainties (e.g., Coles et al. 2011).

The code-bases for TEMPO and TEMPO 2 are different but not fully independent. With appropriate timing model options such that the two programs employed the same algorithms and conventions (using the same clock standards, employing the same solar system ephemeris, using the same obliquity of the ecliptic, excluding a solar wind model (see below), etc.) the fit results were nearly identical between these two programs. The vast majority of all fit parameters agreed to $\lesssim 10 \%$ of their $1 \sigma$ uncertainties, and the ephemeris files we provide are able to be used in both programs. PSR J1713+0747 is an exception, as its complicated timing model includes time-varying orbital geometry terms that are handled slightly differently by each program. In this paper we report only the TEMPO results for all the pulsars.

The number of fit parameters in each timing model depends on the observed spin, astrometric, and environmental properties of the given pulsar. We used ecliptic coordinates to fit for all astrometric parameters in order to reduce parameter covariances, and we fit for parallax for all pulsars, regardless of whether the resulting fit value was physically meaningful (i.e., positive) or significant. We also fit for proper motion for all pulsars except for the two (PSRs J0931-1902 and J1832-0836) which had observing timespans less than one year. The timing models contain fits for spin frequency and its first time-derivative, with higher-order spin noise, if present, being parametrized by the red noise model. We fit five Keplerian binary parameters for all binary pulsars using either the Damour \& Deruelle (1985, "DD") or Lange et al. (2001, "ELL1") binary models. The former is a generally applicable, fully relativistic description of the pulsar's orbit, while the latter

\footnotetext{
33 See http://www.iausofa.org/2015_0209_C/sofa/sofa_ts_c.pdf for a detailed discussion of the various timescale transformations.

34 http://naif.jpl.nasa.gov/naif/
}

is an alternate parameterization that improves numerical stability for very low-eccentricity orbits. We introduced postKeplerian parameters (e.g., Damour \& Taylor 1992) when model accuracy was significantly improved as determined by an $F$-test significance value of 0.0027 (i.e., $3 \sigma$ significant). Information on the timing models, noise models and residual statistics is presented in Table 3 .

We incorporated timing model parameters to describe dispersive delays in the TOAs from the time-variable integrated column of ionized gas between the observatory and each pulsar. These delays are primarily due to the turbulent ISM but also include smaller contributions from the solar wind and the Earth's ionosphere. The delays are characterized by the timedependent DM for each pulsar which is directly proportional to the integrated electron column density. The expected TOA delay for a broadband radio pulse is $\Delta t_{\mathrm{DM}} \propto \mathrm{DM} \nu^{-2}$, where $\nu$ is the observing frequency.

We measured a value of DM at nearly every observing epoch for each pulsar, enumerated using the TEMPO/TEMPO2 parameter "DMX." Since dual-receiver observations were sometimes separated by several days, we allowed a single constant DM value to apply to a window of up to 14 days of observations. These measured DM values include the effects of ionospheric, interstellar, and solar wind dispersion (i.e., the solar wind DM model typically applied by default in TEMPO/TEMPO2 was disabled for this analysis). The best-fit DMX values are shown in the timing summary figures for each pulsar below. The average DM value for each pulsar is highly covariant with profile shape evolution versus frequency (Section 4.2). However, DM variation can be easily distinguished from the constant-in-time profile shape terms. The DM error bars shown in the summary figures represent the uncertainty on the meansubtracted values $\left(\mathrm{DMX}_{i}-\langle\mathrm{DMX}\rangle\right)$, removing the large covariance affecting the mean DM. These uncertainties are determined via an appropriate linear transformation of the original post-fit parameter covariance matrix.

Variations in DM over time are primarily attributed to an evolving view of the line of sight electron column due to the relative motion of the Earth, the pulsar, and the ISM (e.g., Ramachandran et al. 2006). Many pulsars in our data set exhibit slow, long-term DM trends that are at least qualitatively in agreement with the expectation of turbulent electron density structure in the ISM. For several pulsars, annual variations in DM are also apparent. Those at low ecliptic latitude likely have a significant solar wind contribution to their DM-notable examples are J1614-2230 and J2010-1323 with ecliptic latitudes of -1.2 and 6.5 , respectively. These pulsars show a sharp increase in DM at the times of year that they pass behind the Sun. Less sharp, more sinusoidal annual trends are seen in some cases, notably J0613-0200 and J1643-1224. A possible explanation for this is line of sight motion due to Earth's orbit projected onto an ISM density gradient, as discussed by Keith et al. (2013). Using an independent data set, Keith et al. (2013) also detect significant annual DM modulation in several of the same sources where it is apparent in our results. Finally, isolated DM "events" such as the 2008-2009 dip shown by $\mathrm{J} 1713+0747$ are occasionally visible. This event is not yet well explained, but indicates the need for additional study. A more detailed astrophysical analysis of all DM results from this data set is currently underway.

Regardless of the cause, since each pulsar's DM is evolving with time, the fact that our dual-frequency measurements are 
Table 3

Summary of Timing Model Fits

\begin{tabular}{|c|c|c|c|c|c|c|c|c|c|c|c|c|c|}
\hline \multirow[t]{2}{*}{ Source } & \multirow{2}{*}{$\begin{array}{l}\text { Number } \\
\text { of TOAs }\end{array}$} & \multicolumn{6}{|c|}{ Number of Fit Parameters $^{\mathrm{a}}$} & \multicolumn{2}{|c|}{$\mathrm{rms}^{\mathrm{b}}(\mu \mathrm{s})$} & \multicolumn{3}{|c|}{ Red Noise $^{c}$} & \multirow{2}{*}{$\begin{array}{c}\text { Figure } \\
\text { Number }\end{array}$} \\
\hline & & $\mathrm{S}$ & A & B & $\mathrm{DM}$ & FD & $\mathrm{J}$ & Full & White & $A_{\text {red }}$ & $\gamma_{\text {red }}$ & $\log _{10} B$ & \\
\hline $\mathrm{J} 0023+0923$ & 4598 & 3 & 5 & 5 & 28 & 1 & 1 & 0.320 & $\ldots$ & $\ldots$ & $\ldots$ & 1.40 & 7 \\
\hline $\mathrm{J} 0030+0451$ & 2468 & 3 & 5 & 0 & 60 & 0 & 1 & 0.723 & 0.212 & 0.014 & -4.8 & 4.99 & 8 \\
\hline $\mathrm{J} 0340+4130$ & 3008 & 3 & 5 & 0 & 27 & 1 & 1 & 0.385 & $\ldots$ & $\ldots$ & $\ldots$ & 0.01 & 9 \\
\hline J0613-0200 & 7651 & 3 & 5 & 7 & 90 & 2 & 1 & 0.592 & 0.165 & 0.093 & -2.9 & 3.71 & 10 \\
\hline $\mathrm{J} 0645+5158$ & 2896 & 3 & 5 & 0 & 33 & 2 & 1 & 0.052 & $\ldots$ & $\ldots$ & $\ldots$ & -0.08 & 11 \\
\hline J0931-1902 & 719 & 3 & 3 & 0 & 11 & 0 & 1 & 0.381 & $\ldots$ & $\ldots$ & $\ldots$ & -0.07 & 12 \\
\hline $\mathrm{J} 1012+5307$ & 11995 & 3 & 5 & 5 & 95 & 1 & 1 & 1.197 & 0.355 & 0.669 & -1.0 & 14.31 & 13 \\
\hline J1024-0719 & 5073 & 3 & 5 & 0 & 53 & 2 & 1 & 0.280 & $\ldots$ & $\ldots$ & $\ldots$ & 0.08 & 14 \\
\hline J1455-3330 & 5122 & 3 & 5 & 6 & 81 & 1 & 1 & 0.694 & $\ldots$ & $\ldots$ & $\ldots$ & 0.05 & 15 \\
\hline J1600-3053 & 8174 & 3 & 5 & 8 & 74 & 2 & 1 & 0.197 & $\ldots$ & $\ldots$ & $\ldots$ & -0.01 & 16 \\
\hline J1614-2230 & 7517 & 3 & 5 & 7 & 54 & 1 & 1 & 0.189 & $\ldots$ & $\ldots$ & $\ldots$ & 0.02 & 17 \\
\hline $\mathrm{J} 1640+2224$ & 2565 & 3 & 5 & 9 & 65 & 2 & 1 & 0.158 & $\ldots$ & $\ldots$ & $\ldots$ & -0.03 & 18 \\
\hline J1643-1224 & 7119 & 3 & 5 & 6 & 91 & 2 & 1 & 2.057 & 0.331 & $1.231^{\mathrm{d}}$ & -1.7 & 18.33 & 19 \\
\hline $\mathrm{J} 1713+0747$ & 15830 & 3 & 5 & 8 & 106 & 4 & 3 & 0.116 & $\ldots$ & $\ldots$ & $\ldots$ & 0.01 & 20 \\
\hline $\mathrm{J} 1738+0333$ & 2711 & 3 & 5 & 5 & 28 & 1 & 1 & 0.308 & $\ldots$ & $\ldots$ & $\ldots$ & 0.01 & 21 \\
\hline $\mathrm{J} 1741+1351$ & 1600 & 3 & 5 & 8 & 27 & 0 & 1 & 0.103 & $\ldots$ & $\ldots$ & $\ldots$ & -0.02 & 22 \\
\hline J1744-1134 & 9020 & 3 & 5 & 0 & 88 & 2 & 1 & 0.334 & $\ldots$ & $\ldots$ & $\ldots$ & 0.25 & 23 \\
\hline $\mathrm{J} 1747-4036$ & 2778 & 3 & 5 & 0 & 25 & 1 & 1 & 0.531 & $\ldots$ & $\ldots$ & $\ldots$ & 0.12 & 24 \\
\hline $\mathrm{J} 1832-0836$ & 1136 & 3 & 3 & 0 & 10 & 0 & 1 & 0.121 & $\ldots$ & $\ldots$ & $\ldots$ & -0.04 & 25 \\
\hline $\mathrm{J} 1853+1303$ & 1411 & 3 & 5 & 6 & 26 & 0 & 1 & 0.235 & $\ldots$ & $\ldots$ & $\ldots$ & -0.02 & 26 \\
\hline B $1855+09$ & 4071 & 3 & 5 & 7 & 72 & 3 & 1 & 1.339 & 0.505 & 0.017 & -4.9 & 2.87 & 27 \\
\hline $\mathrm{J} 1903+0327$ & 1887 & 3 & 5 & 8 & 36 & 2 & 1 & 1.949 & 0.327 & $0.851^{\mathrm{d}}$ & -2.5 & 2.87 & 28 \\
\hline J1909-3744 & 10697 & 3 & 5 & 8 & 88 & 1 & 1 & 0.079 & $\ldots$ & $\ldots$ & $\ldots$ & 0.72 & 29 \\
\hline $\mathrm{J} 1910+1256$ & 2690 & 3 & 5 & 6 & 45 & 1 & 1 & 1.449 & 0.587 & $0.801^{\mathrm{d}}$ & -1.9 & 5.39 & 30 \\
\hline J1918-0642 & 10035 & 3 & 5 & 7 & 87 & 3 & 1 & 0.340 & $\ldots$ & $\ldots$ & $\ldots$ & -0.02 & 31 \\
\hline $\mathrm{J} 1923+2515$ & 939 & 3 & 5 & 0 & 24 & 1 & 1 & 0.266 & $\ldots$ & $\ldots$ & $\ldots$ & -0.06 & 32 \\
\hline B1937+21 & 9966 & 3 & 5 & 0 & 102 & 5 & 3 & 1.549 & 0.104 & 0.197 & -2.4 & 96.48 & 33 \\
\hline $\mathrm{J} 1944+0907$ & 1724 & 3 & 5 & 0 & 28 & 2 & 1 & 2.442 & 0.331 & $0.860^{\mathrm{d}}$ & -2.8 & 2.35 & 34 \\
\hline $\mathrm{J} 1949+3106$ & 1416 & 3 & 5 & 7 & 16 & 0 & 1 & 0.647 & $\ldots$ & $\ldots$ & $\ldots$ & 0.03 & 35 \\
\hline B1953+29 & 1329 & 3 & 5 & 6 & 24 & 2 & 1 & 4.149 & 0.531 & $0.015^{\mathrm{d}}$ & -6.7 & 2.14 & 36 \\
\hline $\mathrm{J} 2010-1323$ & 8068 & 3 & 5 & 0 & 55 & 1 & 1 & 0.312 & $\ldots$ & $\ldots$ & $\ldots$ & 0.08 & 37 \\
\hline $\mathrm{J} 2017+0603$ & 1589 & 3 & 5 & 7 & 24 & 0 & 2 & 0.073 & $\ldots$ & $\ldots$ & $\ldots$ & 0.01 & 38 \\
\hline $\mathrm{J} 2043+1711$ & 1394 & 3 & 5 & 7 & 23 & 1 & 1 & 0.108 & $\ldots$ & $\ldots$ & $\ldots$ & 0.02 & 39 \\
\hline $\mathrm{J} 2145-0750$ & 7369 & 3 & 5 & 6 & 73 & 2 & 1 & 0.371 & $\ldots$ & $\ldots$ & $\ldots$ & 0.20 & 40 \\
\hline $\mathrm{J} 2214+3000$ & 2624 & 3 & 5 & 5 & 25 & 1 & 1 & 0.319 & $\ldots$ & $\ldots$ & $\ldots$ & 0.06 & 41 \\
\hline $\mathrm{J} 2302+4442$ & 3044 & 3 & 5 & 6 & 27 & 1 & 1 & 0.708 & $\ldots$ & $\ldots$ & $\ldots$ & 0.47 & 42 \\
\hline $\mathrm{J} 2317+1439$ & 2650 & 3 & 5 & 8 & 68 & 3 & 2 & 0.267 & $\ldots$ & $\ldots$ & $\ldots$ & 0.04 & 43 \\
\hline
\end{tabular}

Notes.

${ }^{\mathrm{a}}$ Fit parameters: $\mathrm{S}=$ spin; $\mathrm{B}=$ binary; $\mathrm{A}=$ astrometry; $\mathrm{DM}=$ dispersion measure; $\mathrm{FD}=$ frequency dependence; $\mathrm{J}=$ jump.

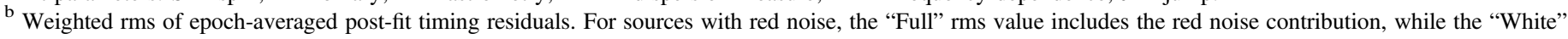
rms does not.

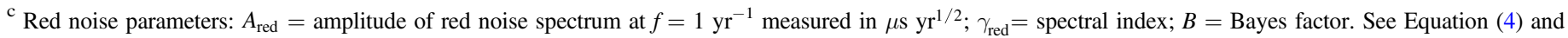
Appendix $\mathrm{C}$ for details.

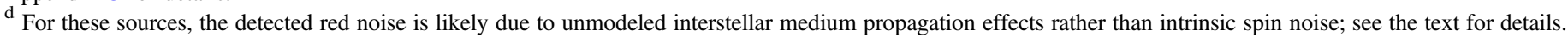

separated by up to 14 days will result in a DM estimation error due to the DM being slightly different at the time of each of the two observations. This effect was studied in detail by Lam et al. (2015), who conclude that 14-day separations can result in timing errors on the order of $\sim 50 \mathrm{~ns}$, depending on the turbulent spectrum of electron density fluctuations in the ISM. In practice this effect will be significantly smaller as the majority of our paired observations are separated by $\lesssim 3$ days (e.g., Lam et al. 2015, Figure 4).

\subsection{Compensation for Frequency Evolution of Pulsar Profiles}

For each pulsar, we calculated TOAs for all frequency channels recorded with a given receiver using a single standard template profile. Because pulse shapes vary with frequency, this produced small systematic FD perturbations in the TOAs in addition to the $\nu^{-2}$ offsets due to dispersion. An example of such common behavior in our data set is in Figure 2. In previous work, we compensated for these FD perturbations for each pulsar by fitting arbitrary time offsets to each spectral channel, thus adding a large number of free parameters to the timing model (Demorest et al. 2013).

For this analysis, we developed a heuristic approach to remove as much of the "FD" bias from our timing residuals as possible by incorporating an additional timing delay $\Delta t_{\mathrm{FD}}$ to all timing models, where

$$
\Delta t_{\mathrm{FD}}=\sum_{i=1}^{n} c_{i} \log \left(\frac{\nu}{1 \mathrm{GHz}}\right)^{i}
$$

and the coefficients $c_{i}$ are fit parameters in the timing models. For any given pulsar, the number of terms needed was 


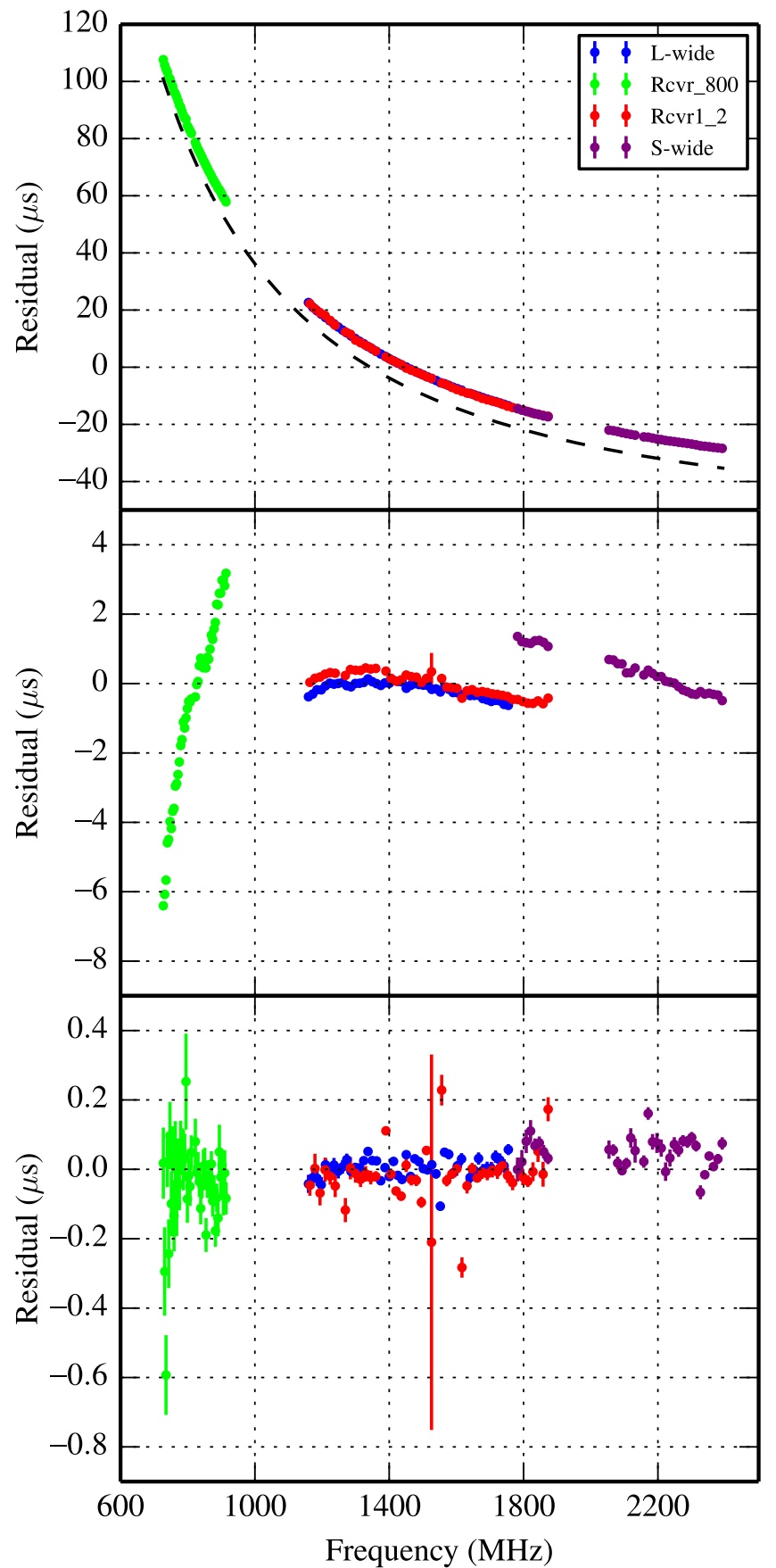

Figure 2. Average timing residual vs. radio frequency for PSR J1713+0747. Upper panel: non-dispersive frequency dependent (FD) residuals with all other timing model parameters held fixed at their best-fit values. The dashed line indicates the best-fit FD model from Equation (2), offset for plot clarity. Middle panel: residuals when FD model is not included in the fit. Lower panel: final residuals when FD model is included in the fit. Note the different $y$-axis scales in each panel. See also a similar presentation of these data by Zhu et al. (2015).

determined by an $F$-test significance value of 0.0027 , the same criterion used for other timing model parameters. The number of parameters used ranged from 0 to 5 (Table 3), and reflects the degree to which profile evolution is important for each pulsar. An illustrative example of the application of our FD model for bias removal is shown in Figure 2 (see also discussion of this approach by Zhu et al. 2015).

This algorithm significantly reduced the number of free parameters in the timing model compared to our previous work, but it remains an ad hoc procedure-it is applied only after TOAs are calculated and does not directly utilize the additional pulse shape information available in the profile data. In future work we plan to explore additional approaches to solving this problem, including TOAs derived from a broadband profilefitting approach that directly incorporates profile evolution versus frequency (Pennucci et al. 2014).

\subsection{Timing Summary}

A basic summary of the timing model fit results, including number of TOAs, number of fit parameters, noise model results, and basic statistics of the residuals is presented in Table 3. The full set of best-fit timing model parameter values, and their associated uncertainties, are publicly available and are distributed along with the TOA data presented here. The models are provided in the standard "par file" format understood by TEMPO and TEMPO2. These results potentially contain a significant amount of astrophysical information about these pulsars, their orbits and binary companions, and the ISM properties along their line of sight, however we have postponed a detailed interpretation of this to future work (see Section 6 for a partial list). It should be noted that while the models presented here provide an accurate description of the time of arrival data for the purposes of gravitational wave detection, certain model parameters (in particular, parallax and Shapiro delay) may require a more sophisticated uncertainty analysis before astrophysically meaningful conclusions can be drawn from their values.

\section{NOISE CHARACTERIZATION}

\subsection{Noise Model}

The noise model used in the analysis is a parameterized one that includes the effects of several noise sources that produce different correlations of TOAs obtained in non-overlapping time blocks and frequency channels. For instance, the template matching errors due to radiometer noise are uncorrelated in both time and frequency, but pulse-jitter noise (Cordes \& Shannon 2010) appears to affect all TOAs obtained simultaneously in different frequency channels. Correlated timing noise with a red power spectrum occurs to varying degree in different pulsars. Spin noise is achromatic and is much smaller in MSPs compared to objects with stronger magnetic fields and longer spin periods. Chromatic red noise due to propagation through intervening plasmas (ISM, interplanetary medium, and ionosphere) may also be present if dispersive delays are not removed perfectly or if scattering and refraction effects contribute significantly. Jitter noise appears to be highly correlated across hundreds of $\mathrm{MHz}$ for those MSPs that have been analyzed in detail (Dolch et al. 2014; Shannon et al. 2014). An approach to noise modeling has been discussed extensively in van Haasteren \& Levin (2013), Ellis (2013, 2014), van Haasteren \& Vallisneri $(2014,2015)$, and Arzoumanian et al. (2014). For more details about the specific noise model and implementation for the current study see Appendix C. Here we will summarize the parameterization used for this data release.

Our model for noise starts with the measurement error on each TOA, $\sigma_{i, k}$, determined by the template-matching TOA calculation algorithm; here $i$ is the TOA number and the subscript $k$ denotes the backend/receiver system. Because such measurement errors may be underestimated, we allow for them 
to be increased by systematic quadrature and scaling factors, determined on a system-by-system basis,

$$
\sigma_{i, k} \rightarrow E_{k}\left(\sigma_{i, k}^{2}+Q_{k}^{2}\right)^{1 / 2},
$$

where $E_{k}$ and $Q_{k}$ are the EFAC and EQUAD parameters used in the TEMPO/TEMPO2 timing code.

In addition to the template-fitting errors, we also include TOA errors that are uncorrelated in time but completely correlated between TOAs obtained at different frequencies measured simultaneously, termed "short-term correlated noise" in Appendix C. The strength of this process is characterized by the ECORR parameter. This term could include true pulse phase jitter (Cordes \& Shannon 2010), known to be present in some pulsars, but can also include other similarly correlated components. Lastly, we model the red noise as a stationary Gaussian process that is parameterized by a power spectrum of the form

$$
P(f)=A_{\text {red }}^{2}\left(\frac{f}{f_{\mathrm{yr}}}\right)^{\gamma_{\mathrm{red}}},
$$

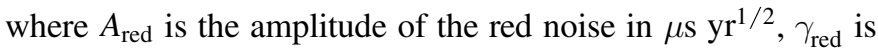
the spectral index, and $f_{\mathrm{yr}}=1 \mathrm{yr}^{-1}$.

These noise parameters are included in a joint likelihood that contains all timing model parameters. For the purposes of this paper, we analytically marginalize over the linear timing model parameters and explore the space of noise parameters via Markov Chain Monte-Carlo (MCMC). We then use the MCMC results to determine the maximum likelihood noise parameters, which are subsequently used as inputs to TEMPO/ TEMPO2 GLS fitting routines. For each pulsar we always include the EFAC, EQUAD, and ECORR parameters for each backend/receiver system. We only include red noise when it is preferred by the data. The red noise model selection is performed with MultiNest (Feroz et al. 2009) using a Bayes factor threshold of 100 to determine whether red noise is included in the final model. The applicable red noise amplitudes, spectral indices, and Bayes factors are shown in Table 3.

\subsection{Noise Analysis}

Here we discuss some of the major features that our noise model reveals. As mentioned above, we only include red noise in the noise model when the data favor its inclusion. In our analysis, ten pulsars meet this criterion and will now be discussed further. Intrinsic pulsar spin noise and its effects have been studied in Blandford et al. (1984), Cordes \& Downs (1985), Arzoumanian et al. (1994), and Shannon \& Cordes (2010, hereafter SC10). Using a sample of both canonical pulsars (CPs) and MSPs, SC10 parameterize the post-fit (after quadratic subtraction) timing noise rms as

$$
\hat{\sigma}_{\mathrm{TN}, 2}=C_{2} \nu^{\alpha}\left|\dot{\nu}_{-15}\right|^{\beta} T_{\mathrm{yr}}^{\gamma},
$$

where $\nu, \dot{\nu}_{-15}$, and $T_{\mathrm{yr}}$ are the spin frequency, spin frequency derivative in units of $10^{-15} \mathrm{~s}^{-2}$, and time span of the data set in years. The best-fit values of the free parameters were found to be $\ln \left(C_{2}\right)=1.6 \pm 0.4, \alpha=-1.4 \pm 0.1, \beta=1.1 \pm 0.1$, and $\gamma=2.0 \pm 0.2$. A fifth parameter, $\delta$, was used to take into account the empirical scatter about the mean relation in Equation (5) and was estimated to be $\delta=1.6 \pm 0.1$ in $\ln \sigma_{\mathrm{TN}, 2}$. First we note that the best fit value of $\gamma$ in Equation (5) corresponds to a power spectral density index of $\gamma_{\text {red }}=$ $-(2 \gamma+1)=-5 \pm 0.4$ in Equation (4). We can estimate $\sigma_{\mathrm{TN}, 2}$ from our noise model by

$$
\begin{aligned}
\sigma_{\mathrm{TN}, 2} & =\left(\int_{1 / T}^{\infty} P(f) d f\right)^{1 / 2} \\
& =2.05 \mathrm{~ns}\left(1-\gamma_{\mathrm{red}}\right)^{-1 / 2}\left(\frac{A_{\mathrm{red}}}{3 \times 10^{-3} \mu \mathrm{sr}^{1 / 2}}\right) T_{\mathrm{yr}}^{\left(1-\gamma_{\mathrm{red}} / 2\right.},
\end{aligned}
$$

where the lower integration limit of $1 / T$ serves as a filter for quadratic subtraction. Furthermore, we can produce a distribution of $\sigma_{\mathrm{TN}, 2}$ by evaluating Equation (6) for all values of $A_{\text {red }}$ and $\gamma_{\text {red }}$ from our MCMC analysis. This will represent our uncertainty in the red noise variance by incorporating the full posterior distributions of red noise parameters as opposed to just the maximum likelihood values.

In essence, SC10 make two predictions for intrinsic pulsar timing spin noise: (i) the red noise spectral index is steep with a value $\sim-5$, and (ii) the red noise rms follows Equation (5) to within a factor of $\exp ( \pm \delta)$. In Figure 3 we show the maximum a-posteriori value and $68 \%$ credible interval for the red noise spectral index, $\gamma_{\text {red }}$, for all pulsars that display significant red noise.

We see that our noise analysis yields a much more shallow spectral index in general than the predicted value of SC10. In fact, of the 10 pulsars that display red noise, only 3 (PSRs $\mathrm{J} 0030+0451, \mathrm{~B} 1953+29$, and B1855+09) have spectral indices consistent with -5 , the others are consistent with $\sim-2$. If we assume that this red noise is due to a random walk in one of the quadratic spin down parameters, then our analysis suggests a random walk in the pulsar phase. ${ }^{35}$ However, it is more likely that in many cases (pulsars marked with an asterisk in Figure 3) this behavior is due to un-modeled ISM effects as we will discuss. In the right panel of Figure 3 we see that our measurements of $\sigma_{\mathrm{TN}, 2}$ are close to $1 \sigma$ consistent with the predictions of SC10 with the exception of PSR B1937+21 which exhibits much weaker red noise than predicted. The gray points show that the $95 \%$ upper limits on $\sigma_{\mathrm{TN}, 2}$ are not consistent for some pulsars. Overall we can state with confidence that our noise analysis is inconsistent with the predictions of SC10 both for the spectral index and the overall red noise rms. To explore this more closely, we will now look into each pulsar in more detail.

PSRs J0030+0451 and B1855+09 are consistent with the spin noise predictions of a steep red noise process. From inspection of Figures 8 and 27 we see that both pulsars are timed for the full nine years and have dual frequency data and $\mathrm{DM}(t)$ corrections for all observing epochs. Furthermore, each set of residuals displays a cubic low frequency term that is characteristic of the predicted steep red process. This appears to be the first evidence of red noise in these pulsars as they have white residuals for both five and six year datasets presented from NANOGrav (Demorest et al. 2013) and the Parkes Pulsar Timing Array (PPTA Manchester et al. 2013).

PSR B1953+29 is also consistent with the spin noise predictions of a steep red noise process. However, as shown in

\footnotetext{
35 As stated in SC10, random walks in the pulsar phase, period and period derivative lead to underlying power spectral indices of $-2,-4$, and -6 , respectively.
} 

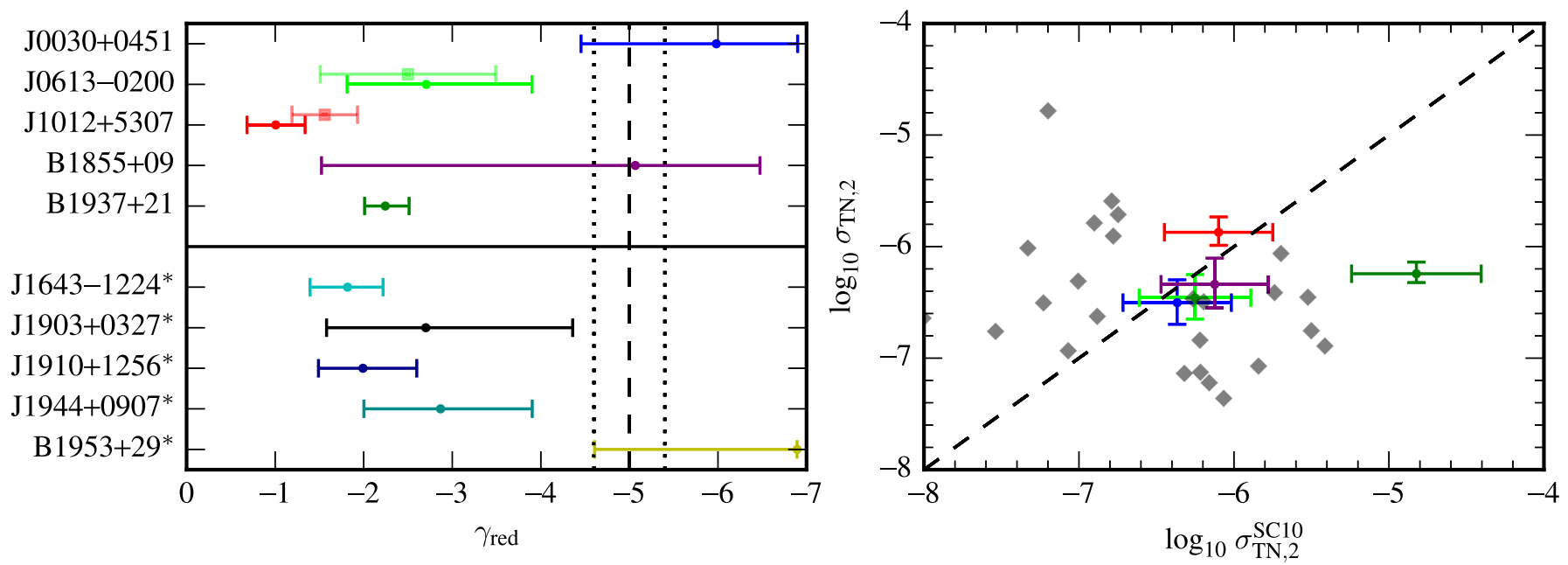

Figure 3. Left panel: maximum a-posteriori value and $68 \%$ credible interval on the red noise spectral index, $\gamma_{\text {red }}$ for all pulsars that display significant red noise. The dashed and dotted black lines represent the mean and $1 \sigma$ predictions on the spectral index from SC10. The points with square markers are the spectral index values presented in Lentati et al. (2015). The pulsars marked with an asterisk indicate those pulsars for which the red noise is likely due to unmodeled interstellar medium propagation effects, rather than intrinsic spin noise. Right panel: measured value for red noise rms for all red pulsars (see the text for details of this calculation) vs. predictions for SC10. The uncertainties are the $1-\sigma$ and $68 \%$ credible region for the predicted and measured values, respectively. The gray points are the $95 \%$ upper limits from the predicted and measured values of $\sigma_{\mathrm{TN}, 2}$ for all other pulsars. The color scheme is the same as the left panel. We do not include the pulsars marked with an asterisk in this figure.

Figure 36, this pulsar lacks dual-frequency data early in the timing campaign, which is likely a strong contributor to the measure red noise.

Both PSRs J1643-1224 and J1910+1256 were identified as displaying strong evidence of red noise in Demorest et al. (2013), and PSR J1643-1224 had a significant $\ddot{\nu}$ in Manchester et al. (2013). In the case of PSR J1643-1224 the shallow red noise process may be due to uncorrected ISM effects that include scattering and refraction (e.g., Foster \& Cordes 1990; Rickett 1990; Cordes \& Shannon 2010)—as can be seen in Figure 19, there is a clear dependence of the noise on radio frequency. The red noise present in the residuals of PSR J1910 +1256 is likely caused by DM variations due to the fact that we only have single-frequency observations for the first four years of the data set. DM variations for a Kolmogorov spectrum would give an $f^{-8 / 3}$ spectrum of TOA variations but this can be altered by linear changes in DM from large-scale structures or changes in pulsar distances from their line of sight motions. This is further indicated by inspection of Figure 30 where the timing residuals appear relatively white after dual frequency observations had begun.

PSR B1937+21 displays the strongest red noise in our sample, consistent with previous work that shows a large amount of red noise (e.g., Shannon \& Cordes 2010; Shannon et al. 2013). Unlike previous work, which indicates a steep, red spectrum our analysis shows a shallower spectrum. Although it has a large cubic trend, the shallow red noise spectral index measurement indicates that there are still high frequency trends in the data. In fact, in Figure 33 we can see that the red noise seems to track the DM changes around 2011.5-2012.5. This feature, along with the large DM $\left(71 \mathrm{pc} \mathrm{cm}^{-3}\right)$, suggest that unmodeled ISM effects may contribute to the observed red noise, particularly at high frequencies, resulting in a lower measured spectral index. An additional explanation for the much shallower spectral index is that we are analyzing only the most recent nine years of data on this pulsar whereas previous analyses have used a much longer time span (SC10 uses up to 24 years of data) not encompassing this new data. This indicates that the noise could be non-stationary in nature. PSR
$\mathrm{J} 1903+0327$ has a similar feature around the same time in which a large drop in the DM coincides with a peak in the red noise. Once again, this effect, in combination with the very large DM (297.6 $\left.\mathrm{pc} \mathrm{cm}^{-3}\right)$ indicate ISM effects as opposed to intrinsic instability as the cause of the red noise.

The measured red noise in PSR J1944+0907 is, likely due to unmodeled DM or scattering/refraction effects since there is only single-frequency data for a large portion of the data set.

As shown in Figure 3, PSRs J0613-0200 and J1012+5307 also display low spectral-index red noise; however, although there are clear high frequency fluctuations in the residuals (Figures 10, 13) there are no obvious radio frequency dependent features present. Therefore, it is difficult to assess the cause of this measured red noise for these pulsars. Nonetheless, these results are very consistent with Lentati et al. (2015; square marker in Figure 3) where a nearly identical noise model was used for these two pulsars observed with EPTA telescopes. This at the very least can rule out any instrumental effects.

It was also pointed out in Manchester et al. (2013) that PSR J1909-3744 displayed some evidence of red noise for the PPTA and EPTA data sets. While our analysis of PSR J1909-3744 did note find sufficient red noise to classify it as a detection (Bayes factor greater than 100; Table 3), the posterior probability distributions for this pulsar hint at the presence of weak red noise, again with a shallow spectral index. This is interesting in that PSR J1909-3744 has very good timing precision and is ideal for GW detection prospects. Future longer data sets will test whether this pulsar truly displays red noise.

Finally, we point out PSRs J1600-3053 and J1747-4036. While not displaying evidence of red noise, we do see nonwhite features in the residuals that are radio frequency dependent. Time varying DM corrections were included for the full range of both of these datasets indicating that while the noise is radio frequency dependent, it is not likely a DM effect.

The noise model used for this data release provides a good fit to the data for most of the pulsars in our data set. However, the model does not accommodate time-variable, chromatic 
phenomena other than DM variations with their $\nu^{-2}$ dependence on radio frequency. Such phenomena may include frequency dependent DMs (Cordes et al. 2015) or scattering, and presently such phenomena are imprecisely absorbed by the red-noise and short-term correlated noise models. As the timespan of wide-band MSP data sets grows, it will become practical to incorporate such phenomena into the noise model.

\section{SUMMARY AND CONCLUSIONS}

In summary, we have obtained, reduced, analyzed, and made public pulse times of arrival for 37 MSPs, using two telescopes, over a time span of up to nine years. A major upgrade in backend instrumentation occurred midway through the data set; we developed a novel method for measuring the instrumental offset between these systems that removes the need to fit this effect using the TOA data. We have continued to develop and refine methods for characterizing time-variable DM and FD pulse shape evolution, while fitting phyiscal timing models to these data. A significant new development is the parameterized noise model presented in this paper, and its inclusion in the timing model fit via a generalized least squares procedure. Our noise modeling has indicated the presence of time-correlated, or red, noise in 10 of these pulsars; we suspect a combination of propagation effects in the ISM and intrinsic spin noise both contribute to these detections, with levels of each varying on a case-by-case basis.

The primary scientific motivation for this project is to detect or limit the presence of nanohertz-frequency gravitational radiation by looking for correlated timing fluctuations among this set of pulsars. While the analysis presented here deals with each pulsar separately, subsequent papers in this series will perform correlation analyses to look for the effect of different gravitational wave signal types. These include the stochastic background from SMBHBs and/or cosmic strings; continuouswave emission from individual binary systems; and gravitational wave bursts with memory following merger events.

In addition to the gravitational wave analyses just mentioned, a number of additional topics are planned to be addressed in future papers, including: detailed investigation of pulse jitter and other sources of noise in these data; measurement of orbital parameters, pulsar and companion masses, and relativistic orbital effects; the effect of scattering on the timing results; pulsar astrometry, distance measurements and kinematics; analysis of the polarization properties of the pulse profiles; flux densities and population analysis; and reanalysis of these data using wide-band timing methodologies. Future improvements to the data set presented here include ongoing increase in the number of pulsars measured, increased cadence on several of the best pulsars in the set, and inclusion of archival Arecibo data covering up to 20 years total timespan.

Author contributions. An alphabetical-order author list was used for this paper in recognition of the fact that a large, decadetimescale project such as this is necessarily the result of the work of many people. All authors contributed to the activities of the NANOGrav collaboration leading to the work presented here, and reviewed the manuscript text and figures prior to the paper's submission. Additional specific contributions to this paper are as follows. Z.A., K.C., P.B.D., T.D., R.D.F., E.F., M.E.G., G.J., M.L.J., M.T.L., L.L., M.A.M., D.J.N., T.T.P., S.M.R., I.H.S., K.S., J.K.S., and W.W.Z. made observations for this project and developed timing models (Section 4). P.B.D. co-developed the
GUPPI and PUPPI instruments, wrote observing proposals, coordinated GBT observations, performed the calibration and TOA generation, developed and implemented the offsetmeasurement analysis, developed and implemented the FD profile evolution model, analyzed TOA accuracy in the lowsignal-to-noise-regime, compiled the data files for public release, coordinated the writing of the paper, and contributed substantially to the text, figures, and tables. J.A.E. developed and implemented the noise model, used it to analyze the present data set, analyzed the red-noise results, co-developed the daily average residual statistic and implemented it for the residual figures, wrote substantial amounts of the text, and contributed tables and figures. D.J.N. co-authored observing proposals, coordinated the NANOGrav Timing Working Group, wrote portions of the text, and contributed a figure and table. T.T.P. undertook careful analysis of the residuals leading to the implementation of the signal-to-noise cutoff. I.H.S. coordinated the Arecibo observations and wrote portions of the text. S.M.R. co-developed the GUPPI and PUPPI instruments and wrote portions of the text. R.v.H. contributed to the noise model and analysis and verified it using independent code and co-developed the daily average residual statistic. E.F. wrote portions of the text. Z.A., J.M.C., T.D., and others edited the manuscript text and/or provided significant input about its content.

The NANOGrav project receives support from National Science Foundation (NSF) PIRE program award number 0968296 and NSF Physics Frontier Center award number 1430284. NANOGrav research at UBC is supported by an NSERC Discovery Grant and Discovery Accelerator Supplement and the Canadian Institute for Advanced Research. Part of this research was carried out at the Jet Propulsion Laboratory, California Institute of Technology, under a contract with the National Aeronautics and Space Administration. T.T.P. is a student at the National Radio Astronomy Observatory. The National Radio Astronomy Observatory is a facility of the NSF operated under cooperative agreement by Associated Universities, Inc. The Arecibo Observatory is operated by SRI International under a cooperative agreement with the NSF (AST-1100968), and in alliance with Ana G. Méndez-Universidad Metropolitana, and the Universities Space Research Association. Some computational work was performed on the Nemo cluster at UWM supported by NSF grant No. 0923409. J.A.E. and R.v.H. acknowledge support by NASA through Einstein Fellowship grants PF4-150120 and PF3-140116, respectively. Y.W. is supported by the National Science Foundation of China under grant No. 11503007.

\section{APPENDIX A TIMING OFFSET DETERMINATION}

A common problem in long-term pulsar timing studies is to connect the timing results between multiple generations of instrumentation at a given telescope. Single-dish pulsar timing data is generally timestamped either at the point where the radio signal is digitized, or somewhat later at the output of a filterbank, when the data are received by software systems. Prior to this point, the signal accumulates additional latency as it passes through various telescope electronics systems. These can include analog cable delays (tens to hundreds of ns), transmission over long fiber optic links (tens of $\mu \mathrm{s}$ ), and filter latencies (up to a few $\mu \mathrm{s}$ ). Since only variations in pulse phase - not its absolute value - are physically meaningful in timing analyses, the presence of time offsets like these is not a problem, as long as they are constant. However, when a new 
backend instrument is added, the delay values (e.g., cabling, filters) typically differ from the previous version. As described in Section 3, this time offset must be accounted for in order to measure long-timescale effects.

A typical approach used in most past timing analyses is to measure offsets between instruments using pulsar TOAs (e.g., Taylor \& Weisberg 1989). An arbitrary offset can be included as an additional term (known as a "JUMP" in TEMPO/TEMPO2) in a timing model fit (see Section 4). Although this is a straightforward approach, it has several drawbacks: first, in the presence of unmodeled red noise, this can introduce systematic biases in other parameters (see for example discussion in Coles et al. 2011); this can be mitigated via improved noise modeling as described in Section 5 and references therein. Next, even if the noise is properly modeled, the offset will be covariant with other long-term effects, most critically with low-frequency gravitational waves, reducing sensitivity to these effects. A refinement to this is to restrict the offset measurement to only a shorter, overlapped span of data between the two instruments, measure it in a separate fit procedure, and hold the resulting value fixed in the main timing model fit. This will reduce covariance with longterm effects, but raises concern that the effect of the offset fit may not be fully accounted for in the other model parameter uncertainties. In both cases, the precision of the measurement is limited by the relevant TOA uncertainties. It is sometimes possible to transfer an offset measurement done using one bright pulsar to other sources, although there may then be concerns about potential systematics, for example due to pulse shape evolution with frequency, calibration inaccuracies, or different instrument configurations used for different sources.

An alternate approach was used recently by Manchester et al. (2013), wherein a locally generated pulsed signal was injected into the common signal path and used to recover the offsets between different systems with much higher precision than could be achieved using astronomical signals. This mitigates all of the problems of TOA-based approaches described above. The only drawbacks are that it requires additional specialpurpose hardware be built and installed at the observatory, and that it can not be applied retroactively-once an instrument has been decommissioned it is no longer possible to perform this measurement. In contrast, simultaneous (or at least contemporaneous) pulsar data is often still available in archival data sets long after the relevant instruments are gone.

We have developed a new method that addresses many of the shortcomings described above. This is based on the fact that for observations that are simultaneous in both time and frequency (i.e., where a single signal is split and fed into multiple backend systems), both instruments see not only the same pulsar signal, but also the same system noise. This correlated noise can be used to recover a time offset with much higher precision than is possible from TOA-based methods. TOA determination can be viewed as a matched filtering process designed to recover the template profile shape. By construction, this filters out a large fraction of the noise that could otherwise be used for an offset measurement. In our method, rather than cross-correlating measured pulse profiles with a noise-free template, pairs of simultaneous pulse profiles from each instrument are cross-correlated with each other. In contrast with TOA measurement described in Section 3, it is advantageous to average the profiles as little as possible prior to this step, to preserve more (correlated) noise. With the profile data at its original time and frequency resolution, we compute
Table 4

Measured Instrumental Offsets ${ }^{\mathrm{a}}$

\begin{tabular}{lccc}
\hline \hline $\begin{array}{l}\text { Receiver } \\
\text { System }\end{array}$ & $\begin{array}{c}\text { Cross-corr } \\
\text { Offset (ns) }\end{array}$ & $\begin{array}{c}\text { J1713+0747 } \\
\text { JUMP (ns) }\end{array}$ & $\begin{array}{r}\text { J1909-3744 } \\
\text { JUMP (ns) }\end{array}$ \\
\hline Arecibo 327 & $785(19)$ & $\ldots$ & $\ldots$ \\
Arecibo 430 & $789(5)$ & $\ldots$ & $\ldots$ \\
Arecibo L-wide & $839(3)$ & $820(75)$ & $\ldots$ \\
Arecibo S-wide & $846(6)$ & $885(82)$ & $\ldots$ \\
GBT Rcvr_800 & $897(8)$ & $951(124)$ & $936(42)$ \\
GBT Rcvr1_2 & $693(3)$ & $599(86)$ & $651(55)$ \\
\hline
\end{tabular}

Note.

${ }^{a}$ Numbers in parentheses are uncertainties in the last digit quoted.
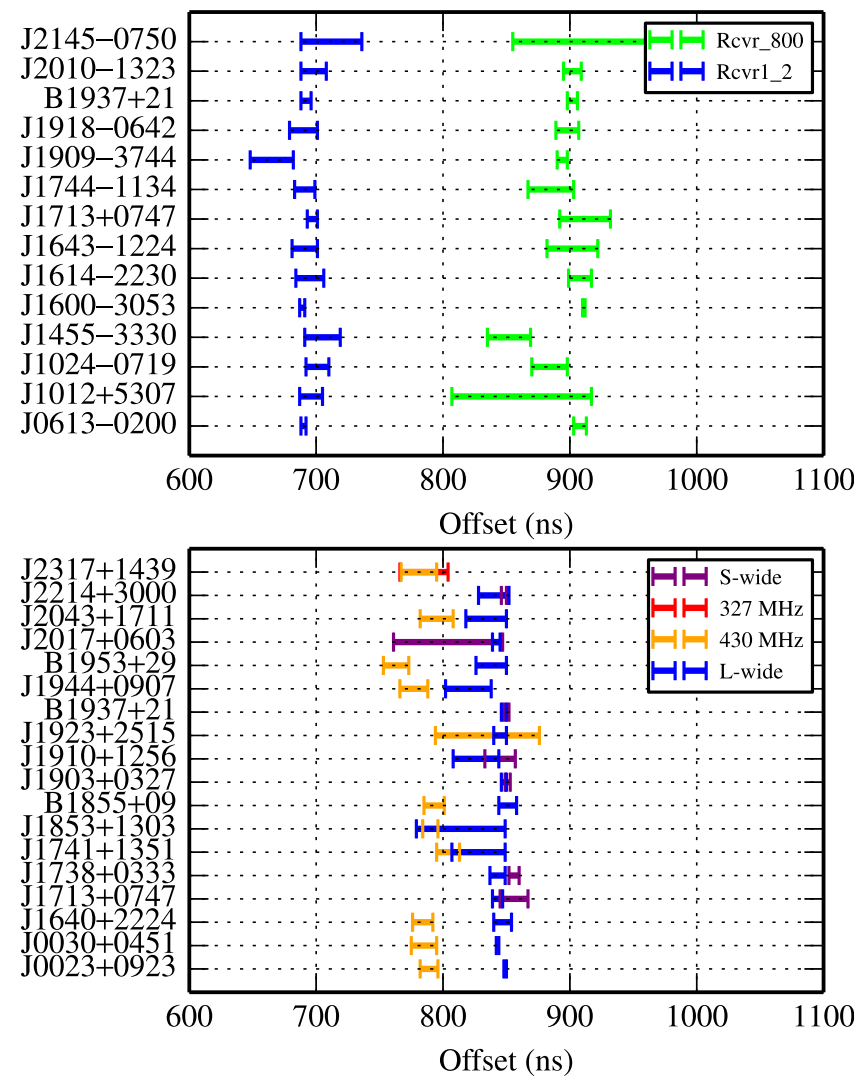

Figure 4. Measured instrumental offsets vs. pulsar for the GBT (top) and Arecibo (bottom).

cross-correlations between all pairs of profiles that overlap in both time and frequency. The cross-correlations from all simultaneous profile pairs for a given pulsar, instrument setup, and epoch, are then averaged together (with a weight proportional to the amount of time-frequency overlap) to form a final correlation function. The lag for which this is maximized results in an offset estimate for that portion of the data. We use this set of individual offset measurements to determine an uncertainty on the average offset, and look for systematic trends as a function of pulsar, time, or instrument setup.

For this work, we are interested in the offset between GASP and GUPPI at the GBT and the offset between ASP and PUPPI at Arecibo. We analyzed all available simultaneously collected profiles with overlapping frequency bands from these pairs of instruments. The results of this offset analysis are shown in Table 4 and Figure 4. After accounting for all a priori 

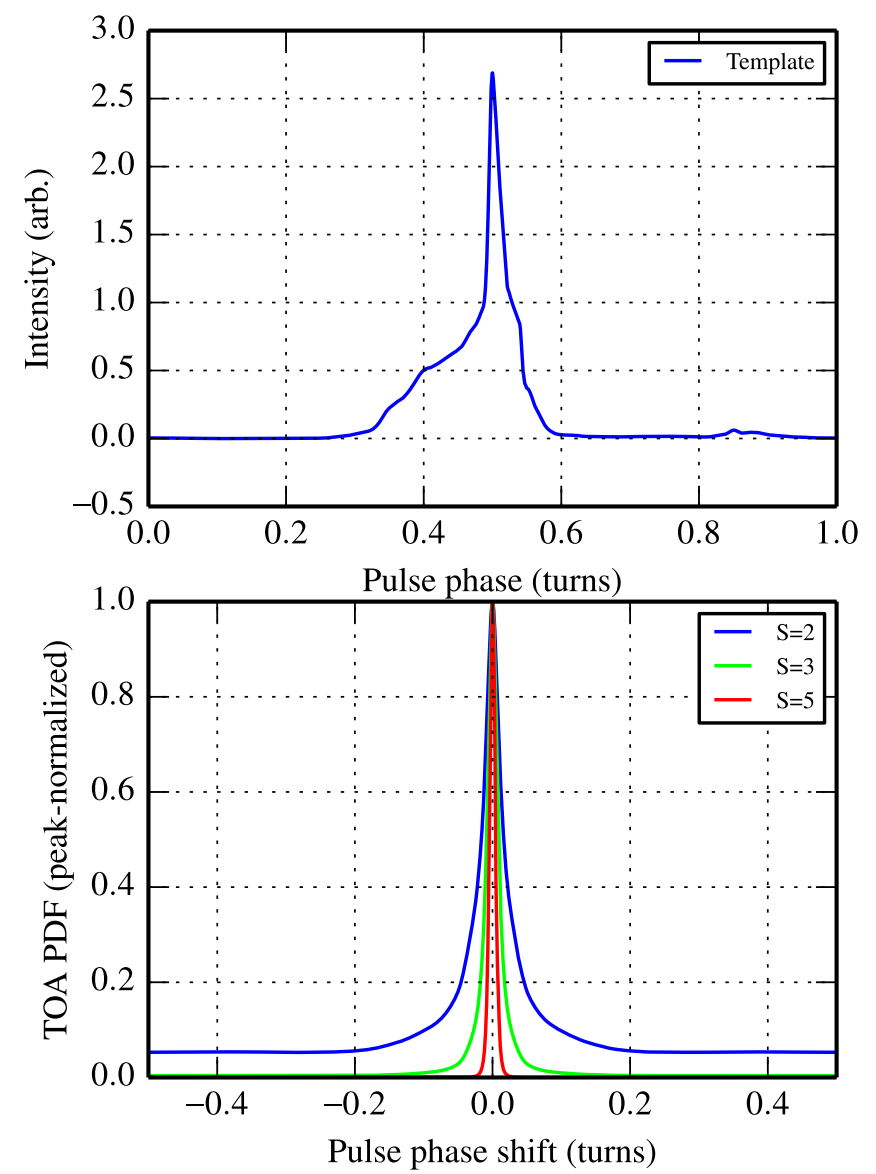

Figure 5. Upper panel: $1.4 \mathrm{GHz}$ template profile for J1455-3330. Lower panel: expected pulse phase shift distributions for several values of $\mathrm{S} / \mathrm{N}$, from Equation (13). For plot clarity these are normalized to 1 at $\phi=0$, rather than to constant integrated area. This shows the evolution of the distribution from nearly Gaussian at higher $\mathrm{S} / \mathrm{N}(S=5)$ to clearly nonGaussian at low-S/N $(S=2)$.

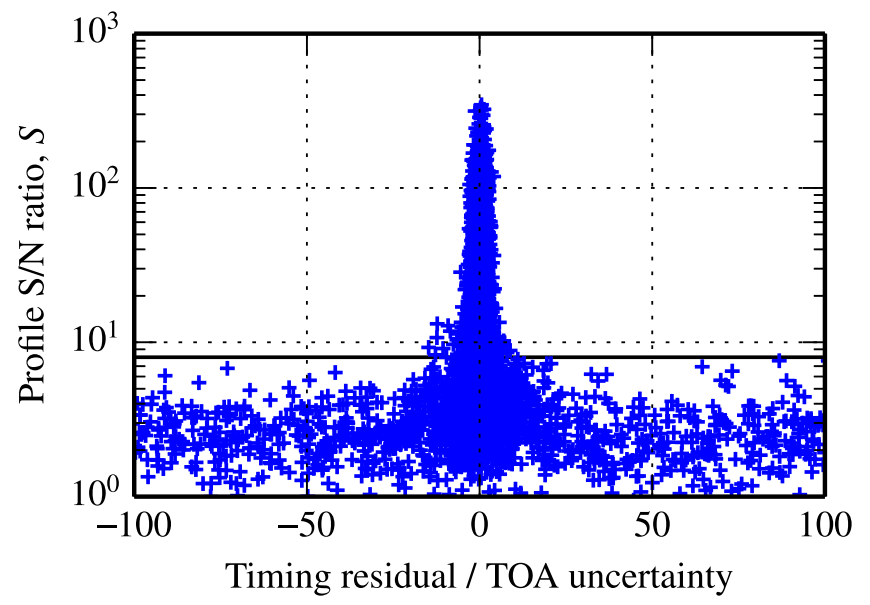

Figure 6. Signal-to-noise ratio $S$ vs. normalized timing residual (residual divided by TOA uncertainty) for J1455-3330 L-band data. All data points below the solid line at $S=8$ were removed from the timing analysis.

predictable latencies in the backend systems, there remains $\sim 700-900 \mathrm{~ns}$ additional offset between ASP/GASP and GUPPI/PUPPI that varies only as a function of signal path and instrument bandwidth. Table 4 lists the average value of all data available for each receiver system, while Figure 4 shows the same data averaged separately for each pulsar. At Arecibo, we obtain consistent results for the 327 and $430 \mathrm{MHz}$ setups, as well as for the L-wide and S-wide setups. This result is expected since these pairs of setups share common analog signal paths and PUPPI bandwidth $(100 \mathrm{MHz}$ and $800 \mathrm{MHz}$ respectively). At Green Bank, the $820 \mathrm{MHz}$ and $1.4 \mathrm{GHz}$ receivers have different signal paths to GUPPI, and the instrument is run at different bandwidth $(200$ and $800 \mathrm{MHz})$. It is likely that both of these factors contribute to the observed offset difference. The sign of these values is such that ASP/ GASP pulses arrive later-the offsets must be subtracted from these TOAs to align them with the GUPPI/PUPPI data. As can be seen in Figure 4, consistent values are obtained from all pulsars for a given receiver system, therefore in our timing analysis we have applied the overall-average (Table 4) values to the TOAs. In our data set this is provided via a time offset ("-to") flag on each TOA line.

As a check on these results, we performed a standard timing analysis on the overlapping TOAs of two pulsars, in each case fitting for an offset between the TOAs from the two different instruments used as part of the timing solution. The results are shown in Table 4, where they are labeled JUMP (the TEMPO parameter used for this offset measurement). These values illustrate that our noise correlation provides both a consistent and much more precise result. For most other pulsars, TOA uncertainties are larger than for the pulsars used in Table 4, hence the JUMP uncertainties are larger as well.

\section{APPENDIX B \\ TOAS IN THE LOW-S/N LIMIT}

In the very low $\mathrm{S} / \mathrm{N}$ regime, the standard template matching procedure breaks down, producing underestimated TOA uncertainties. In addition, the distribution of TOAs in this regime becomes significantly non-Gaussian. Here we derive expressions for the expected TOA probability distribution, and motivate our choice of $\mathrm{S} / \mathrm{N}$ cutoff for TOAs. The use of a $\mathrm{S} / \mathrm{N}$ or TOA uncertainty cutoff, or simply "by-eye" removal of outlier residuals, is often done in pulsar timing analyses. The discussion in this section provides a somewhat more rigorous and quantitative justification for this practice. The behavior of TOA uncertainties in the low-S/N limit was previously explored empirically by Hotan et al. (2005) using simulated data, who reach similar conclusions to what we present here.

We follow the standard Fourier-domain least squares TOA determination approach of Taylor (1992; see also Demorest 2007), writing the expression for $\chi^{2}$ as a function of fitted amplitude $a$ and pulse phase shift $\phi$ as

$$
\begin{aligned}
\chi^{2}(a, \phi) & =\sum_{k} \frac{\left|d_{k}-a t_{k} e^{-2 \pi i k \phi}\right|^{2}}{\sigma^{2}} \\
& =\frac{D^{2}+a^{2} T^{2}-2 a C_{d t}(\phi)}{\sigma^{2}},
\end{aligned}
$$

where these terms come from the discrete Fourier transform of the measured pulse profile $\left(d_{k}\right)$ and template profile $\left(t_{k}\right)$ :

$$
\begin{aligned}
D^{2} & \equiv \sum_{k}\left|d_{k}\right|^{2} \\
T^{2} & \equiv \sum_{k}\left|t_{k}\right|^{2}
\end{aligned}
$$




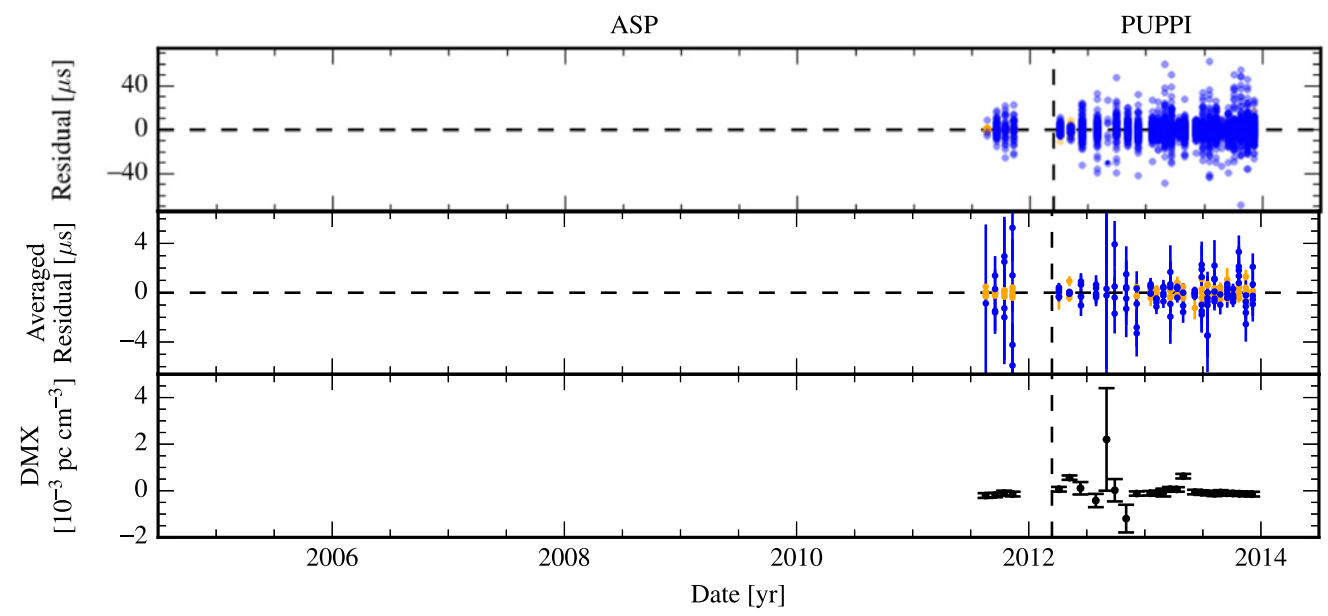

Figure 7. Timing summary for PSR J0023+0923. Colors are blue: $1.4 \mathrm{GHz}$, purple: $2.3 \mathrm{GHz}$, green: $820 \mathrm{MHz}$, orange: $430 \mathrm{MHz}$, red: $327 \mathrm{MHz}$. In the top panel, individual points are semi-transparent; darker regions arise from the overlap of many points.

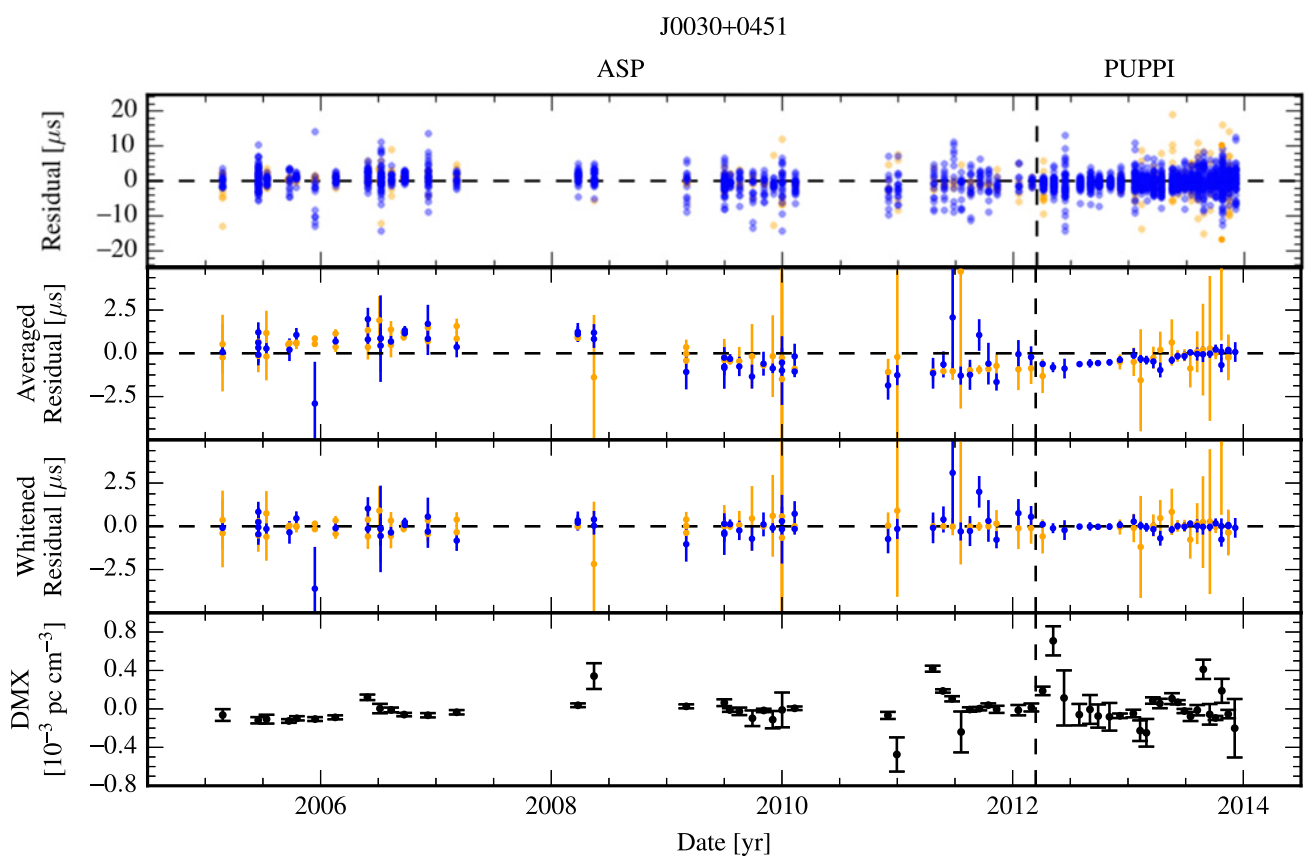

Figure 8. Timing summary for PSR J0030+0451. Colors are blue: $1.4 \mathrm{GHz}$, purple: $2.3 \mathrm{GHz}$, green: $820 \mathrm{MHz}$, orange: $430 \mathrm{MHz}$, red: $327 \mathrm{MHz}$. In the top panel, individual points are semi-transparent; darker regions arise from the overlap of many points.

$$
C_{d t}(\phi) \equiv \mathfrak{R} \sum_{k} d_{k} t_{k}^{*} e^{2 \pi i k \phi}
$$

Here $\sigma^{2}$ is the noise level in each bin of $d_{k}$, and the sum is over pulse harmonics, not including the constant (DC) term. All pulse phase information is contained in the $C_{d t}$ term, illustrating why TOA determination is sometimes described as a cross-correlation between the data and template profiles - the minimum $\chi^{2}$ is always achieved at the phase shift giving maximum crosscorrelation. With the assumption of additive Gaussian noise (implicit in a $\chi^{2}$ fit), the TOA likelihood function is

$p(d \mid a, \phi) \propto e^{-\frac{1}{2} \chi^{2}(a, \phi)}=\exp \left(\frac{2 a C_{d t}(\phi)-D^{2}-a^{2} T^{2}}{2 \sigma^{2}}\right)$, and with the use of uniform priors on $a$ and $\phi$, the posterior distribution is simply proportional to the likelihood, $p(a, \phi \mid d) \propto p(d \mid a, \phi)$. For TOA determination, $a$ is a nuisance parameter, which can be analytically marginalized over in the above expression to get the posterior $\phi$ distribution

$$
p(\phi \mid d) \propto \exp \left(\frac{C_{d t}^{2}(\phi)}{2 \sigma^{2} T^{2}}\right)
$$

By making the substitution $d_{k} \rightarrow a t_{k}$-i.e., the data profile is simply a scaled copy of the template-we can explore the expected shape of these distributions independent of any particular (noisy) data realization. In this case Equation (12) 


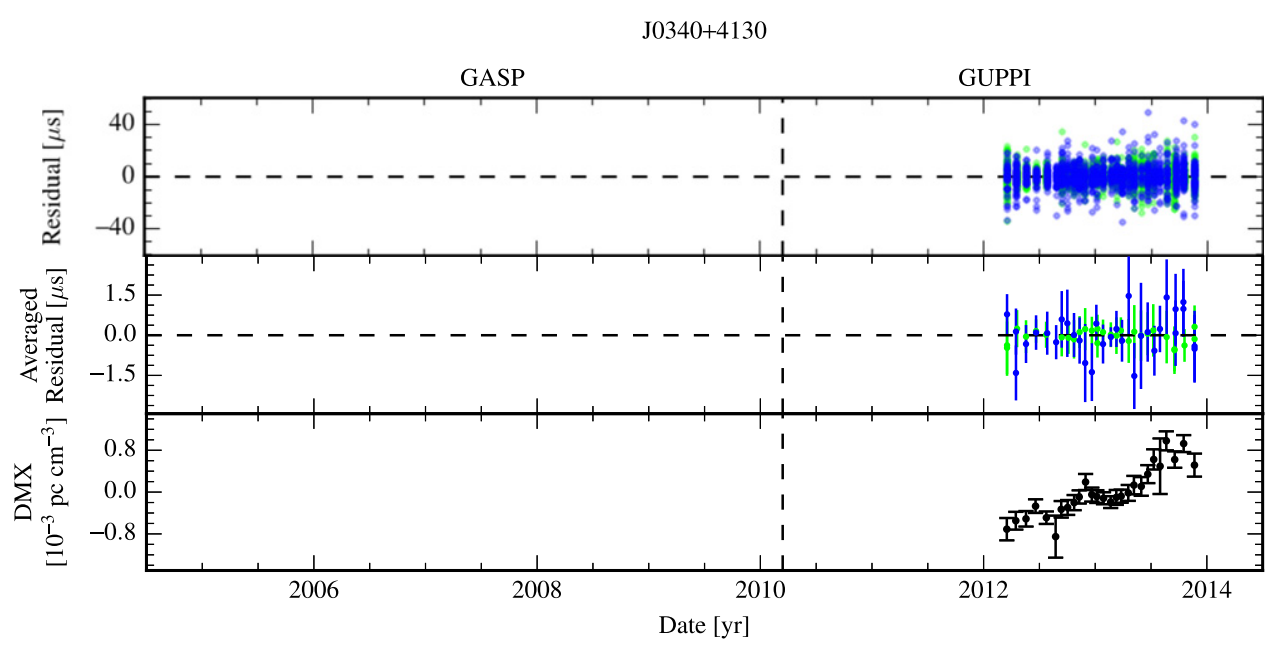

Figure 9. Timing summary for PSR J0340+4130. Colors are blue: $1.4 \mathrm{GHz}$, purple: $2.3 \mathrm{GHz}$, green: $820 \mathrm{MHz}$, orange: $430 \mathrm{MHz}$, red: $327 \mathrm{MHz}$. In the top panel, individual points are semi-transparent; darker regions arise from the overlap of many points.

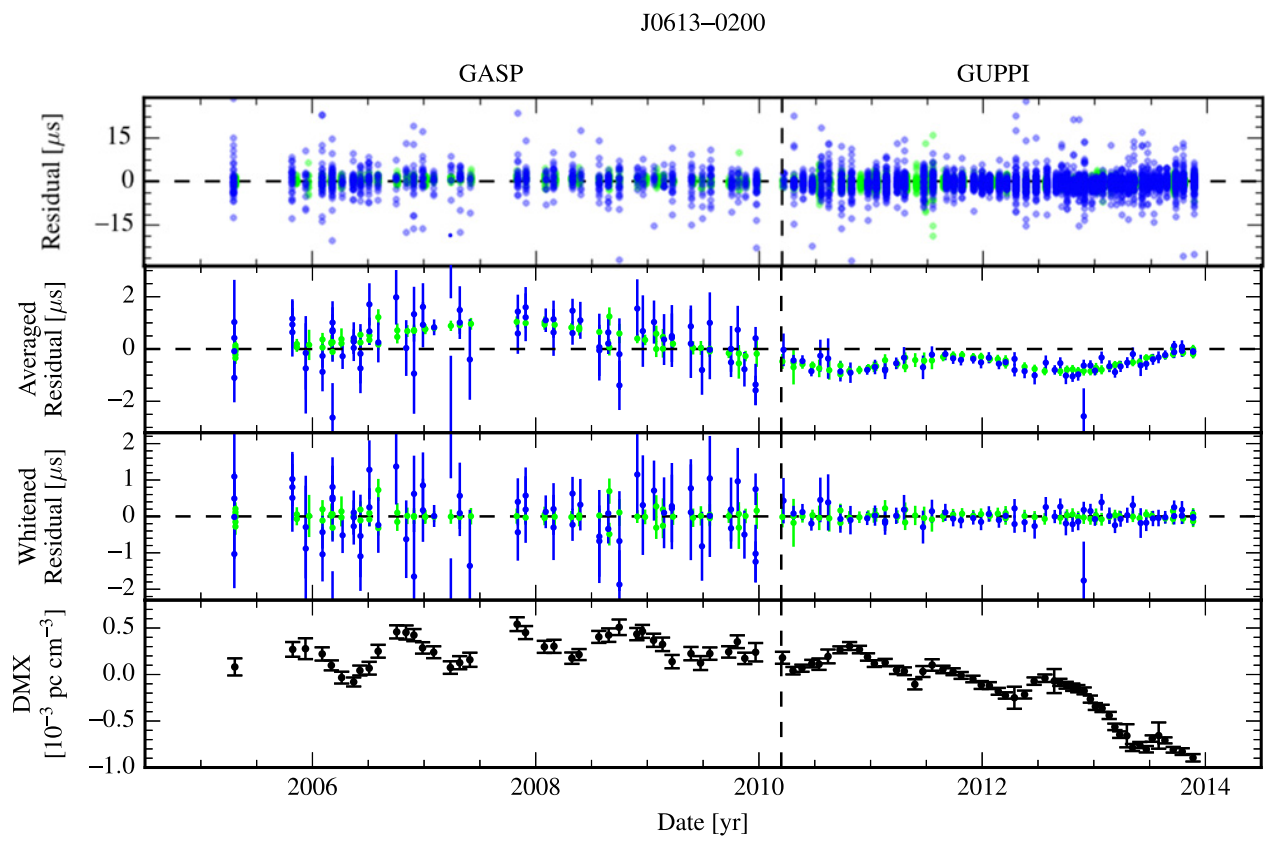

Figure 10. Timing summary for PSR J0613-0200. Colors are blue: $1.4 \mathrm{GHz}$, purple: $2.3 \mathrm{GHz}$, green: $820 \mathrm{MHz}$, orange: $430 \mathrm{MHz}$, red: $327 \mathrm{MHz}$. In the top panel, individual points are semi-transparent; darker regions arise from the overlap of many points.

becomes

$$
p(\phi) \propto \exp \left(\frac{S^{2}}{2} \frac{C_{t t}^{2}(\phi)}{T^{4}}\right)
$$

where $S \equiv a T / \sigma$ defines the $\mathrm{S} / \mathrm{N}$ of the data, and $C_{t t}$ is the template profile's autocorrelation (with normalization $\left.C_{t t}(0)=T^{2}\right)$. For non-detections $(S \rightarrow 0$ limit $), p(\phi)$ becomes a uniform distribution across one turn of phase. For high-S/N detections $(S \gtrsim 10), p(\phi)$ becomes extremely well approximated by a Gaussian, with standard deviation given by the usual template-matching TOA uncertainty formula

$$
\sigma_{\phi}=S^{-1} T\left(C_{t t}^{\prime \prime}(0)\right)^{-1 / 2} \text {. }
$$

In the low-S/N regime between these two limits, the standard uncertainty formula underestimates the true spread of TOA values and signifcant non-Gaussianity is present. We illustrate this in Figures 5 and 6 using data from PSR J1455-3330. This pulsar provides a clear demonstration of this effect, because its wide scintillation bandwidth and moderate average flux result in profiles with a large range of $\mathrm{S} / \mathrm{N}$ values in our data set. If included in a standard $\chi^{2}$-based timing model fit, the low-S $/ \mathrm{N}$ points appear as outliers and have a disproportionately large impact on the results. While it would be possible to mitigate this by a modification of the TOA uncertainties or use of a timing model likelihood based on Equation (12), in our data set the amount of additional information gained from these data points is likely to be marginal at best. Instead, for the work 
J0645+5158

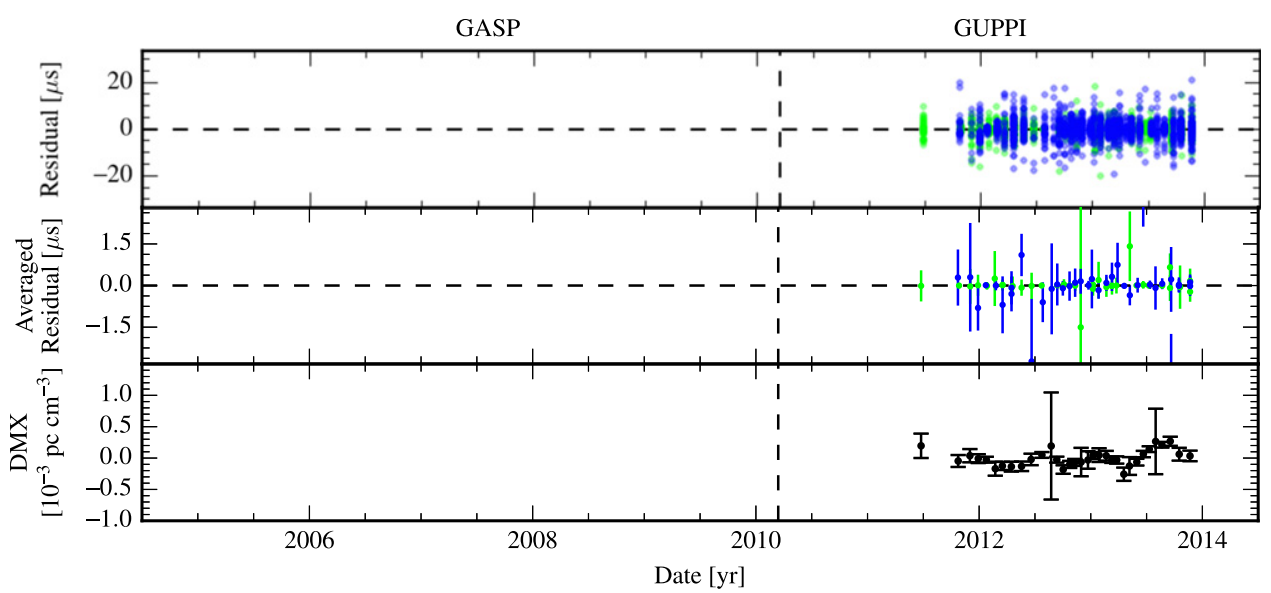

Figure 11. Timing summary for PSR J0645+5158. Colors are blue: $1.4 \mathrm{GHz}$, purple: $2.3 \mathrm{GHz}$, green: $820 \mathrm{MHz}$, orange: $430 \mathrm{MHz}$, red: $327 \mathrm{MHz}$. In the top panel, individual points are semi-transparent; darker regions arise from the overlap of many points.

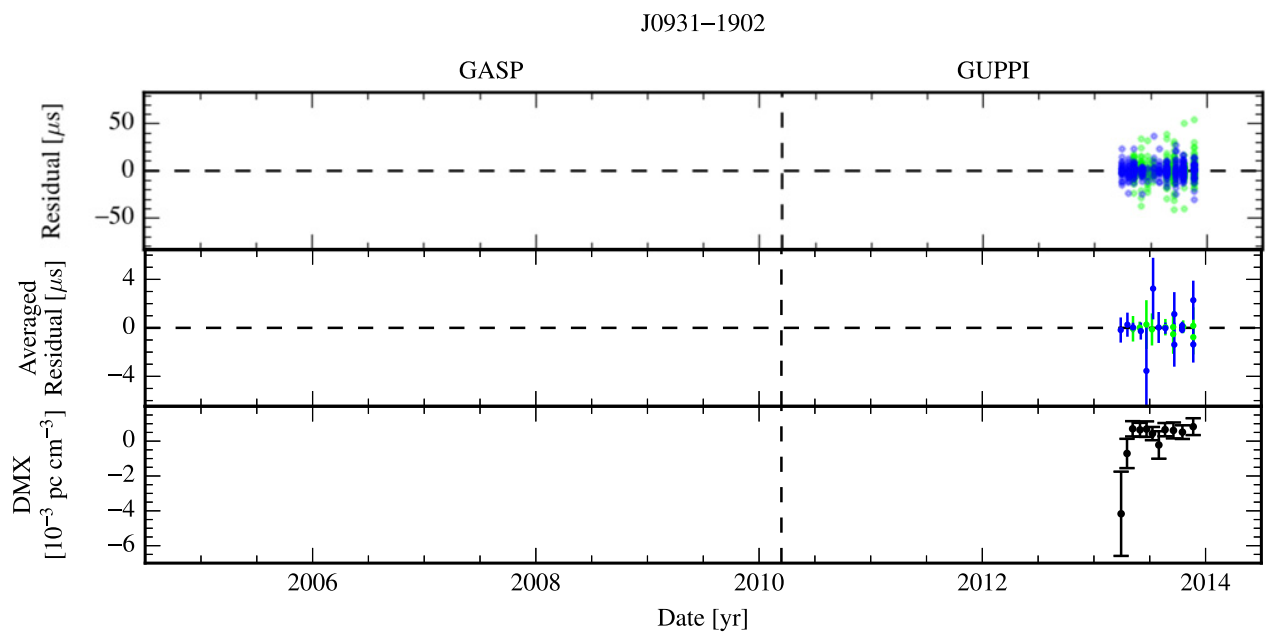

Figure 12. Timing summary for PSR J0931-1902. Colors are blue: $1.4 \mathrm{GHz}$, purple: $2.3 \mathrm{GHz}$, green: $820 \mathrm{MHz}$, orange: $430 \mathrm{MHz}$, red: $327 \mathrm{MHz}$. In the top panel, individual points are semi-transparent; darker regions arise from the overlap of many points.

presented in this paper we have simply removed all TOAs with $S<8$.

\section{APPENDIX C DERIVATION OF NOISE MODEL LIKELIHOOD}

We begin by forming a set of residuals via the standard weighted least squares fitting routine. An $N_{\mathrm{TOA}}$ length vector of residuals can be modeled mathematically as the sum of several deterministic and stochastic sources as follows

$$
\delta \boldsymbol{t}=M \boldsymbol{\epsilon}+F \boldsymbol{a}+U \boldsymbol{j}+\boldsymbol{n} .
$$

The first term on the right hand side $(M \epsilon)$ describes small deterministic trends due to timing model subtraction. Here $M$ is the timing model design matrix and $\epsilon$ is a vector of small timing model parameter offsets. Next, the term $F \boldsymbol{a}$ models the red noise via a Fourier decomposition ${ }^{36}-F$ is the Fourier design matrix that has columns of alternating sine and cosine functions for frequencies in the range $\left[1 / T, n_{\text {mode }} / T\right] ; T$ is the total observation time span, $\Delta f=1 / T$, and $n_{\text {mode }}$ is the

\footnotetext{
$\overline{36}$ The Fourier basis was chosen to improve computational efficiency; it is not a requirement of this noise modeling method.
}

number of frequencies included in the sum. The vector $\boldsymbol{a}$ gives the amplitudes of the Fourier basis functions (see Arzoumanian et al. 2014; Lentati et al. 2014, for more details). The term $U j$ describes noise that is uncorrelated in time but completely correlated between TOAs obtained at different frequencies measured simultaneously. This term could be due to pulse phase jitter but could also have other components not due to jitter. This term characterizes noise that is completely correlated for all TOAs in a given time bin but completely uncorrelated between time bins. The matrix $U$ is an $N_{\text {TOA }} \times N_{\text {tb }}$ matrix that maps TOAs to a given time bin and $\boldsymbol{j}$ is the amplitude of the short time-scale fluctuations. Finally the last term $\boldsymbol{n}$ describes a Gaussian white noise process that characterizes time-, and frequency-independent random noise left in the data.

Since the white noise is modeled as Gaussian, the likelihood function for the noise is given by

$$
p(\boldsymbol{n})=\frac{\exp \left(-\frac{1}{2} \boldsymbol{n}^{T} N^{-1} \boldsymbol{n}\right)}{\sqrt{\operatorname{det}(2 \pi N)}},
$$




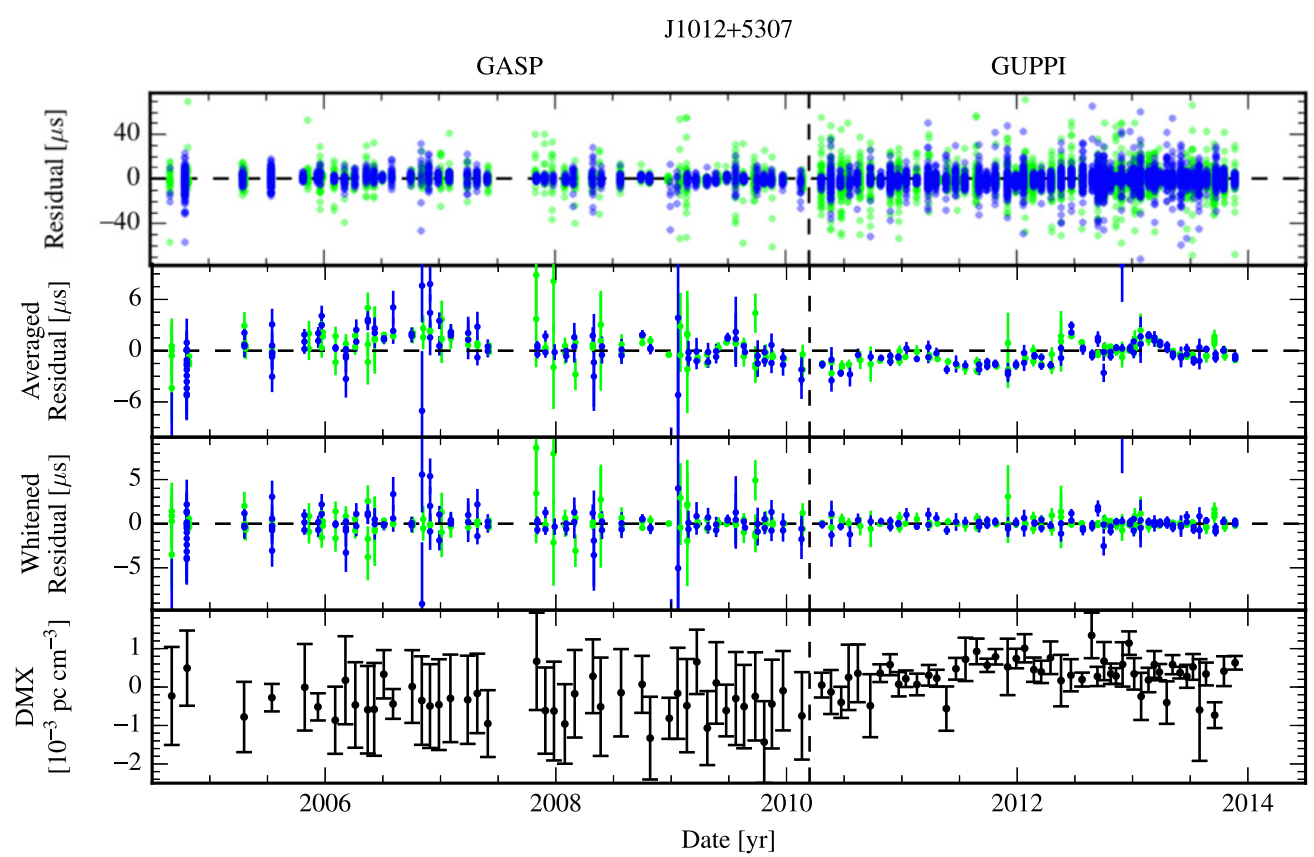

Figure 13. Timing summary for PSR J1012+5307. Colors are blue: $1.4 \mathrm{GHz}$, purple: $2.3 \mathrm{GHz}$, green: $820 \mathrm{MHz}$, orange: $430 \mathrm{MHz}$, red: $327 \mathrm{MHz}$. In the top panel, individual points are semi-transparent; darker regions arise from the overlap of many points.

J1024-0719

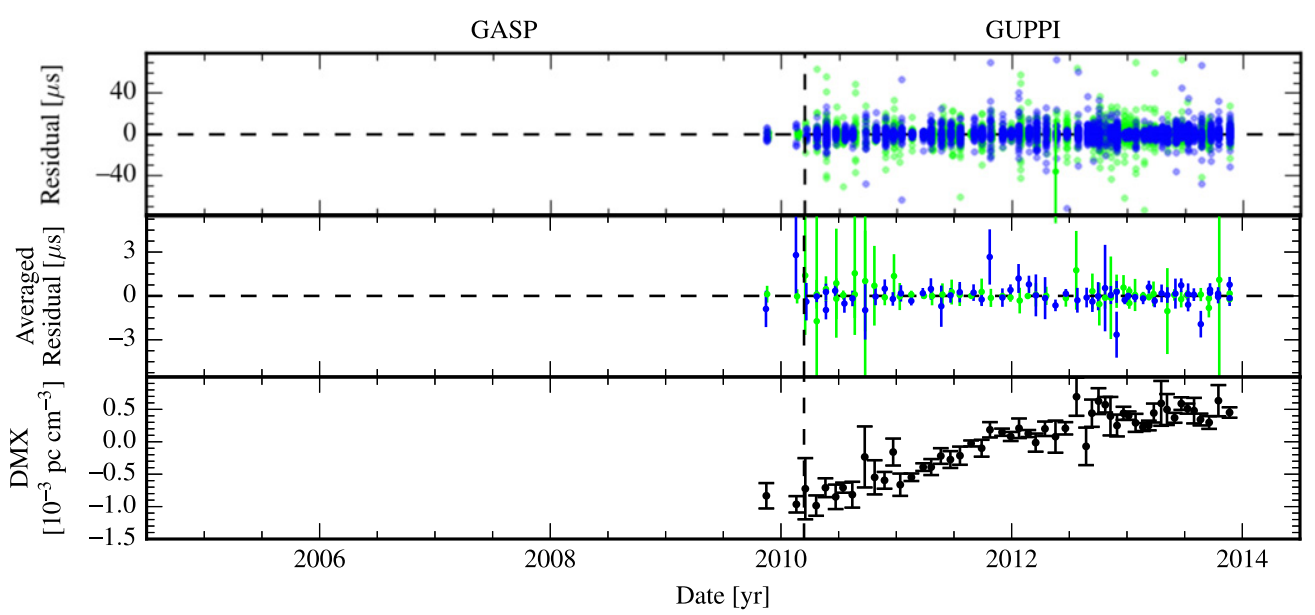

Figure 14. Timing summary for PSR J1024-0719. Colors are blue: $1.4 \mathrm{GHz}$, purple: $2.3 \mathrm{GHz}$, green: $820 \mathrm{MHz}$, orange: $430 \mathrm{MHz}$, red: $327 \mathrm{MHz}$. In the top panel, individual points are semi-transparent; darker regions arise from the overlap of many points.

where

$$
N_{i j, k}=E_{k}^{2}\left(W_{i j}+Q_{k}^{2} \delta_{i j}\right)
$$

is an $N_{\mathrm{TOA}} \times N_{\mathrm{TOA}}$ matrix with $E_{k}$ and $Q_{k}$ corresponding to TEMPO and TEMPO2's EFAC and EQUAD parameters for each observing backend, respectively, $W=\operatorname{diag}\left\{\sigma_{i}^{2}\right\}$ is a $\operatorname{diag-}$ onal matrix of TOA uncertainties, and $\delta_{\mathrm{ij}}$ is the Kronecker delta function. The notation is such that the matrix elements $(i, j)$ apply to those TOAs corresponding to the backend observing system labeled by $k$. We can now write the likelihood function of the residuals as

$$
p(\delta \boldsymbol{t} \mid \boldsymbol{\epsilon}, \boldsymbol{a}, \boldsymbol{j}, \phi)=\frac{\exp \left(-\frac{1}{2} \boldsymbol{r}^{T} N^{-1} \boldsymbol{r}\right)}{\sqrt{\operatorname{det}(2 \pi N)}},
$$

where $\phi$ denotes the $E_{k}$ and $Q_{k}$ parameters and

$$
\boldsymbol{r}=\delta \boldsymbol{t}-M \boldsymbol{\epsilon}-\boldsymbol{F a}-U \boldsymbol{j} .
$$

We now wish to impose prior distributions on our short timescale correlated noise and red noise. Both can be modeled as Gaussian processes by imposing the following priors

$$
\begin{aligned}
p\left(\boldsymbol{j} \mid J_{k}\right)= & \frac{\exp \left(-\frac{1}{2} \boldsymbol{j}^{T} \mathcal{J}^{-1} \boldsymbol{j}\right)}{\sqrt{\operatorname{det}(2 \pi \mathcal{J})}} \\
p\left(\boldsymbol{a} \mid \rho_{n}\right)= & \frac{\exp \left(-\frac{1}{2} \boldsymbol{a}^{T} \varphi^{-1} \boldsymbol{a}\right)}{\sqrt{\operatorname{det}(2 \pi \varphi)}},
\end{aligned}
$$

where $\mathcal{J}_{i j, k}=J_{k}^{2} \delta_{i j}$ is an $N_{\mathrm{tb}} \times N_{\mathrm{tb}}$ matrix with diagonal elements, and $\mathrm{J}_{k}^{2}$ describes the variance of the jitter-like 


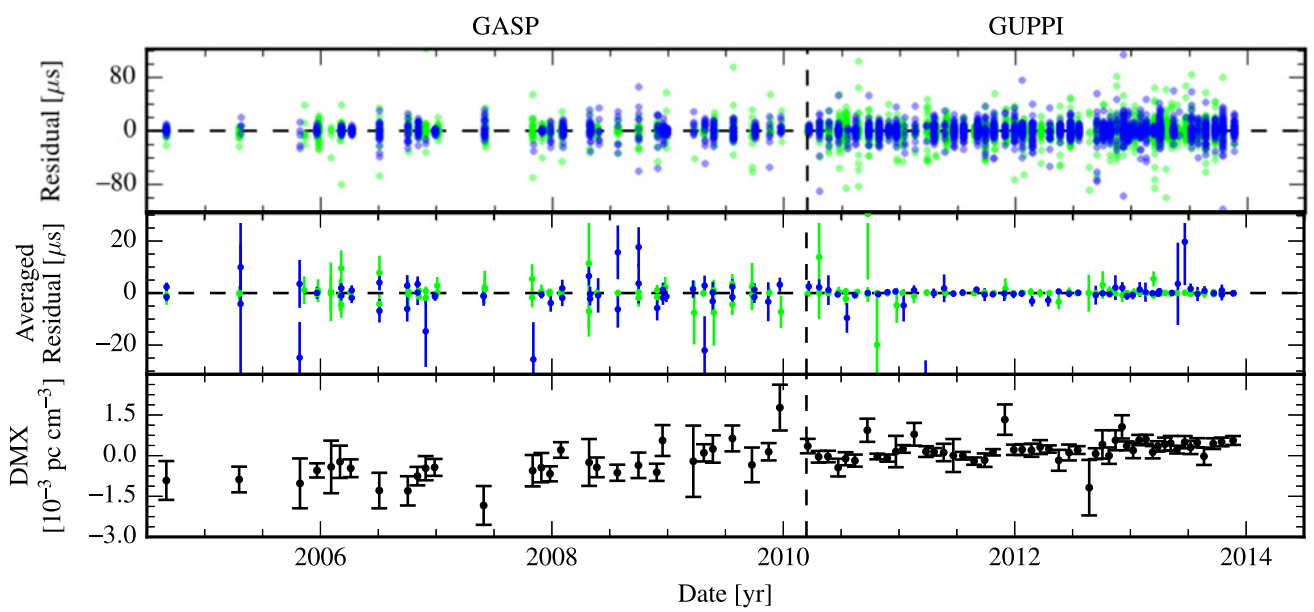

Figure 15. Timing summary for PSR J1455-3330. Colors are blue: $1.4 \mathrm{GHz}$, purple: $2.3 \mathrm{GHz}$, green: $820 \mathrm{MHz}$, orange: $430 \mathrm{MHz}$, red: $327 \mathrm{MHz}$. In the top panel, individual points are semi-transparent; darker regions arise from the overlap of many points.

J1600-3053

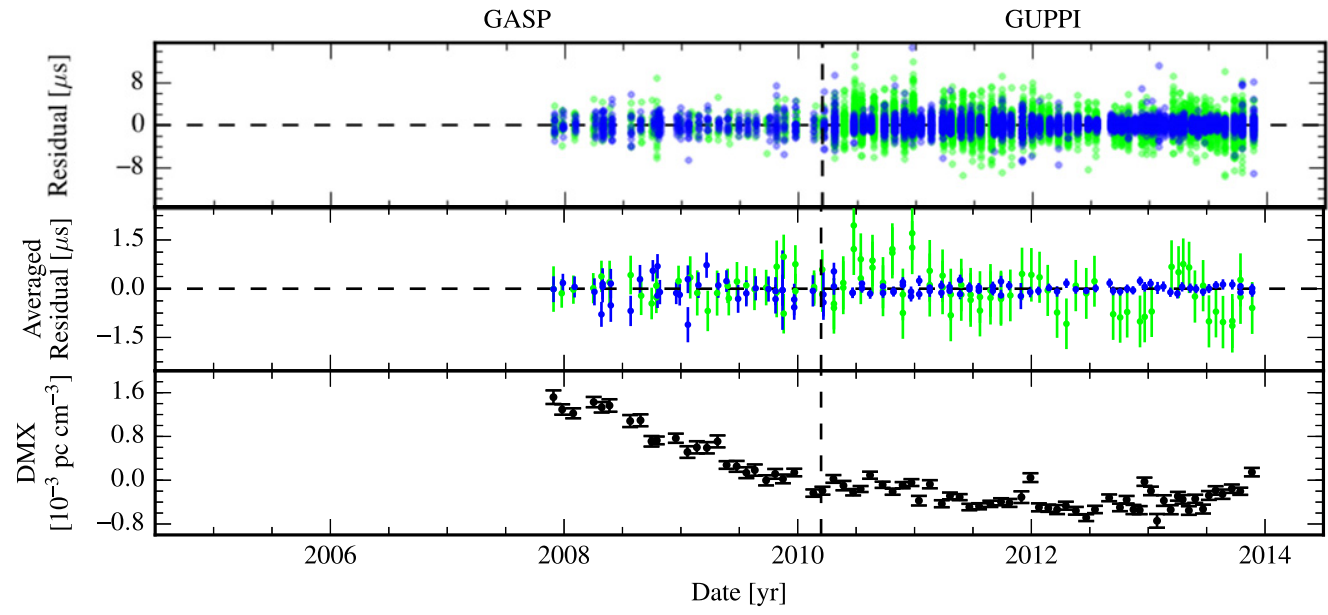

Figure 16. Timing summary for PSR J1600-3053. Colors are blue: $1.4 \mathrm{GHz}$, purple: $2.3 \mathrm{GHz}$, green: $820 \mathrm{MHz}$, orange: $430 \mathrm{MHz}$, red: $327 \mathrm{MHz}$. In the top panel, individual points are semi-transparent; darker regions arise from the overlap of many points.

J1614-2230

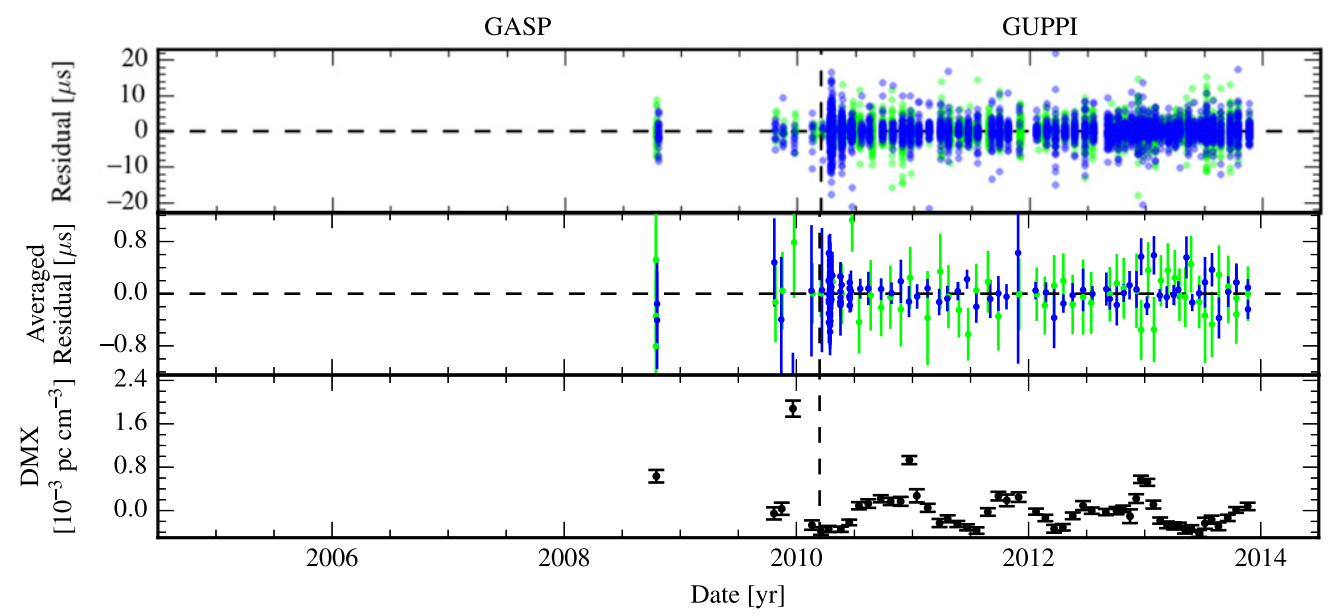

Figure 17. Timing summary for PSR J1614-2230. Colors are blue: $1.4 \mathrm{GHz}$, purple: $2.3 \mathrm{GHz}$, green: $820 \mathrm{MHz}$, orange: $430 \mathrm{MHz}$, red: $327 \mathrm{MHz}$. In the top panel, individual points are semi-transparent; darker regions arise from the overlap of many points. 


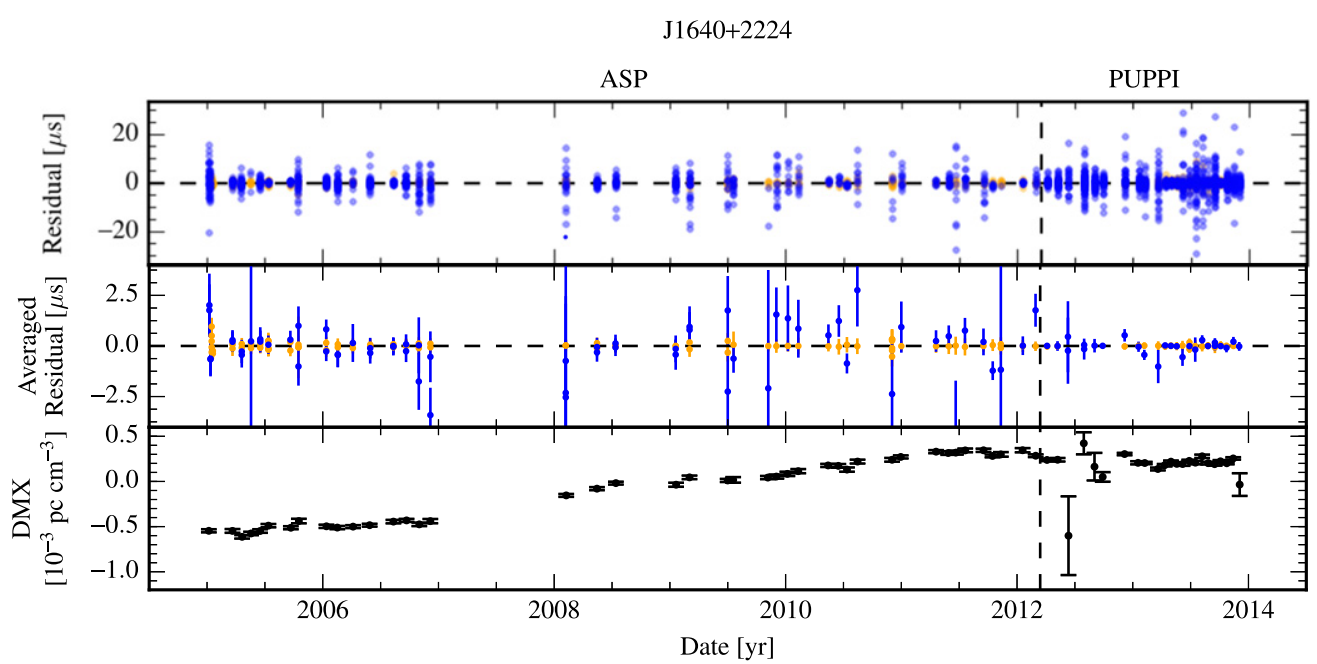

Figure 18. Timing summary for PSR J1640+2224. Colors are blue: $1.4 \mathrm{GHz}$, purple: $2.3 \mathrm{GHz}$, green: $820 \mathrm{MHz}$, orange: $430 \mathrm{MHz}$, red: $327 \mathrm{MHz}$. In the top panel, individual points are semi-transparent; darker regions arise from the overlap of many points.

J1643-1224

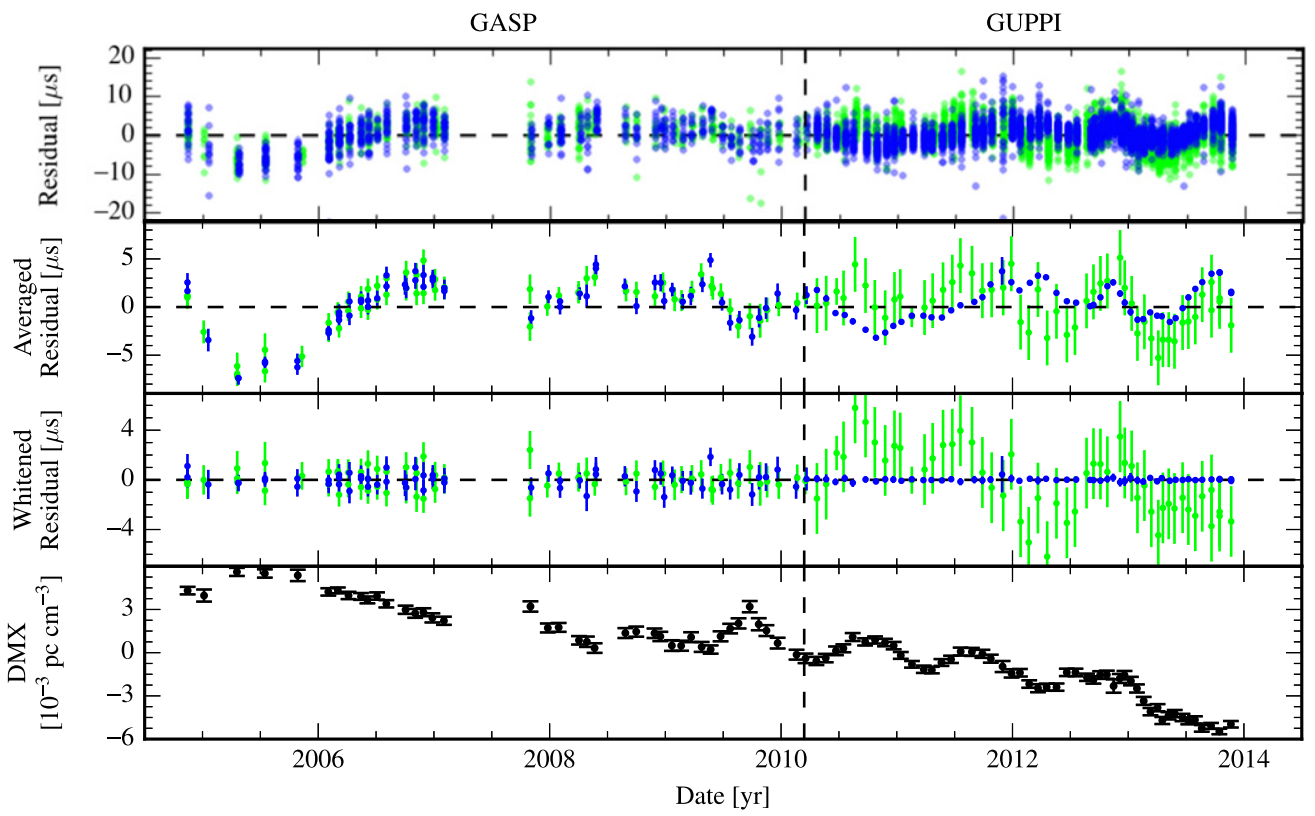

Figure 19. Timing summary for PSR J1643-1224. Colors are blue: $1.4 \mathrm{GHz}$, purple: $2.3 \mathrm{GHz}$, green: $820 \mathrm{MHz}$, orange: $430 \mathrm{MHz}$, red: $327 \mathrm{MHz}$. In the top panel, individual points are semi-transparent; darker regions arise from the overlap of many points.

correlated noise for each observing backend; it is also referred to as the ECORR parameter in TEMPO and TEMPO2. Furthermore $\varphi_{i j}=\operatorname{diag}\left\{10^{\rho_{n}}\right\}$ is an $2 n_{\text {mode }} \times 2 n_{\text {mode }}$ matrix describing the variance of the red noise Fourier coefficients at each frequency. In this framework, the coefficients of the $\varphi$-matrix are related to the power spectral density evaluated at a given frequency. In principle we could use the power spectrum coefficients, $10^{\rho_{n}}$, themselves as free parameters but in this work we parameterize them via a power law

$$
\varphi_{n} \equiv 10^{\rho_{n}}=\frac{1}{T_{\text {span }}} A_{\text {red }}^{2}\left(\frac{f_{n}}{f_{\mathrm{yr}}}\right)^{\gamma_{\text {red }}}
$$

where $T_{\text {span }}$ is the total observation time, $A_{\text {red }}$ is the amplitude of

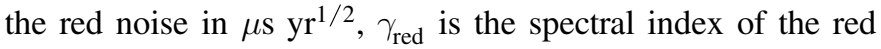
noise, $f_{\mathrm{yr}}$ is the reference frequency of $1 \mathrm{yr}^{-1}$, and $f_{n}$ is the $n$th Fourier frequency assuming Nyquist sampling. We see that the prior distributions on jitter-like correlated noise and red noise are themselves parameterized by some combination of hyperparameters. We can write down the posterior distribution for the residuals

$$
p(\boldsymbol{\epsilon}, \boldsymbol{a}, \boldsymbol{j}, \boldsymbol{\phi} \mid \delta \boldsymbol{t}) \propto p(\delta \boldsymbol{t} \mid \boldsymbol{\epsilon}, \boldsymbol{a}, \boldsymbol{j}, \boldsymbol{\phi}) p\left(\boldsymbol{j} \mid J_{k}\right) p\left(\boldsymbol{a} \mid \rho_{n}\right) .
$$

For the purposes of estimating the underlying noise characteristics of our data set, the parameters $\boldsymbol{\epsilon}, \boldsymbol{j}$, and $\boldsymbol{a}$ are 


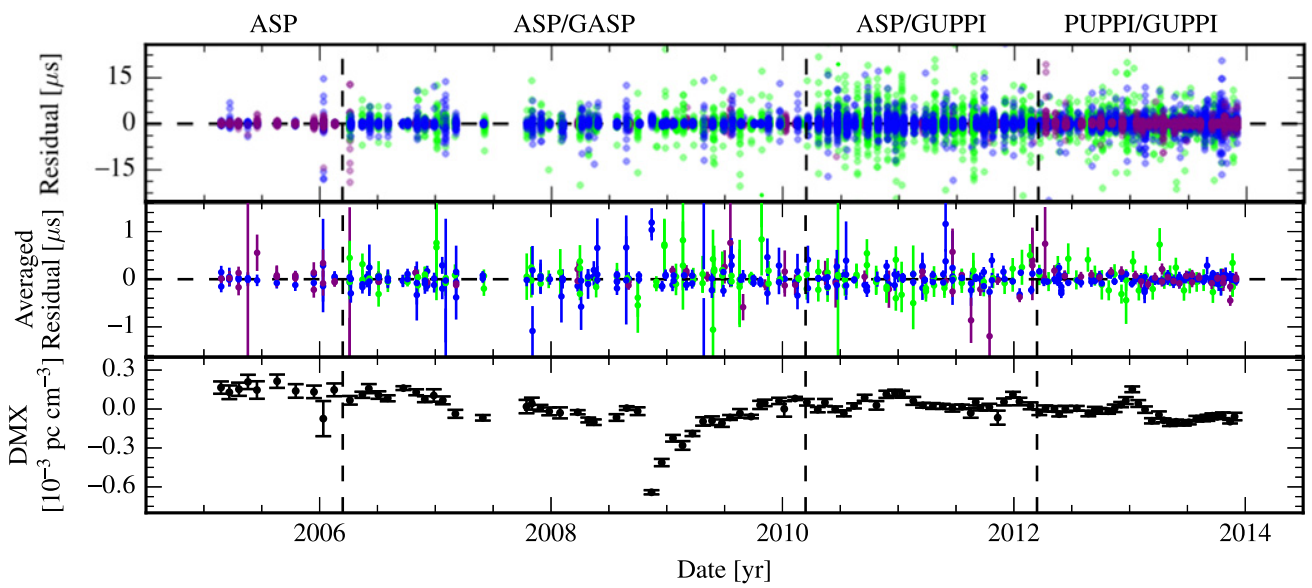

Figure 20. Timing summary for PSR J1713+0747. Colors are blue: $1.4 \mathrm{GHz}$, purple: $2.3 \mathrm{GHz}$, green: $820 \mathrm{MHz}$, orange: $430 \mathrm{MHz}$, red: $327 \mathrm{MHz}$. In the top panel, individual points are semi-transparent; darker regions arise from the overlap of many points.

$\mathrm{J} 1738+0333$

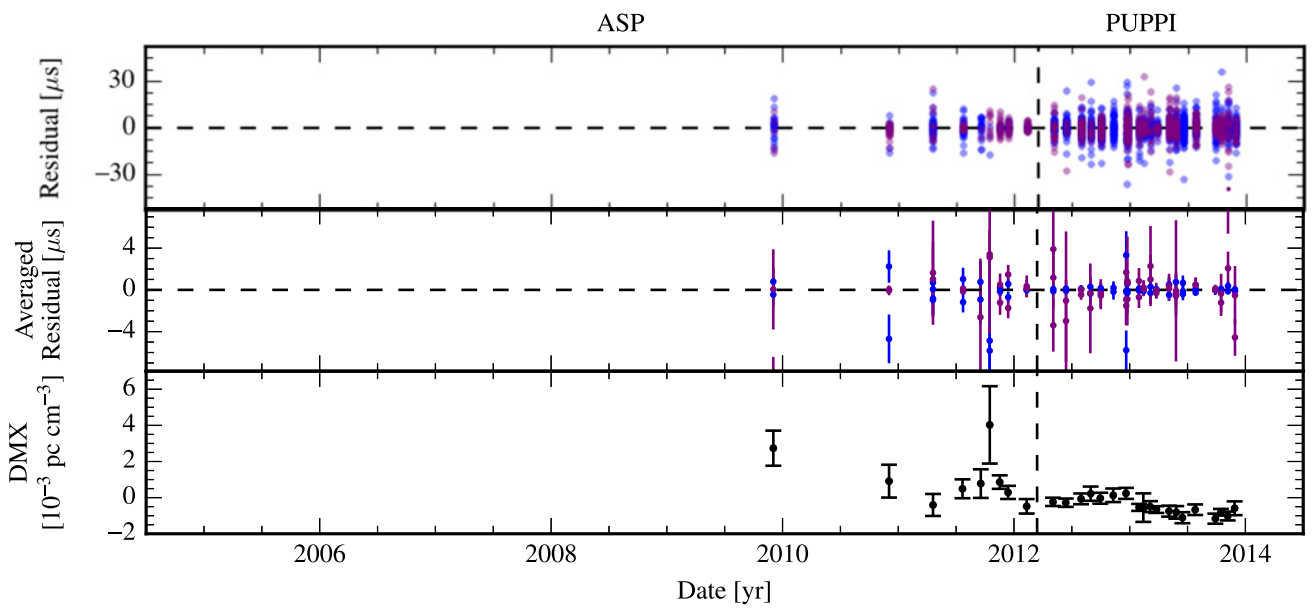

Figure 21. Timing summary for PSR J1738+0333. Colors are blue: $1.4 \mathrm{GHz}$, purple: $2.3 \mathrm{GHz}$, green: $820 \mathrm{MHz}$, orange: $430 \mathrm{MHz}$, red: $327 \mathrm{MHz}$. In the top panel, individual points are semi-transparent; darker regions arise from the overlap of many points.

\section{$\mathrm{J} 1741+1351$}

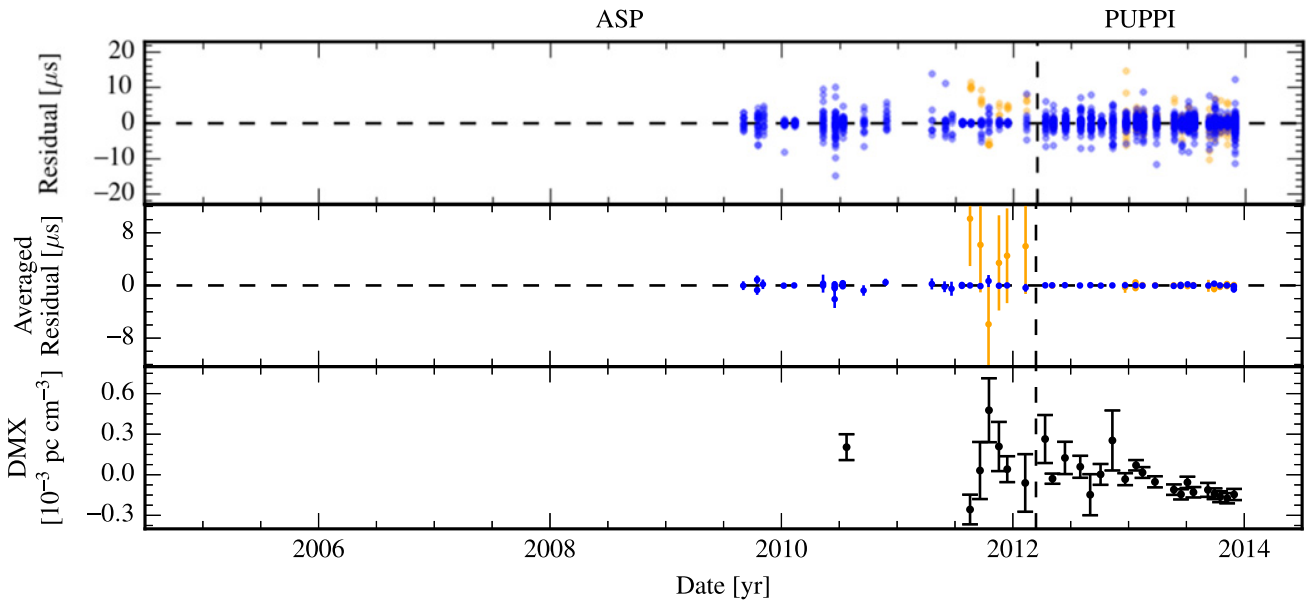

Figure 22. Timing summary for PSR J1741+1351. Colors are blue: $1.4 \mathrm{GHz}$, purple: $2.3 \mathrm{GHz}$, green: $820 \mathrm{MHz}$, orange: $430 \mathrm{MHz}$, red: $327 \mathrm{MHz}$. In the top panel, individual points are semi-transparent; darker regions arise from the overlap of many points. 


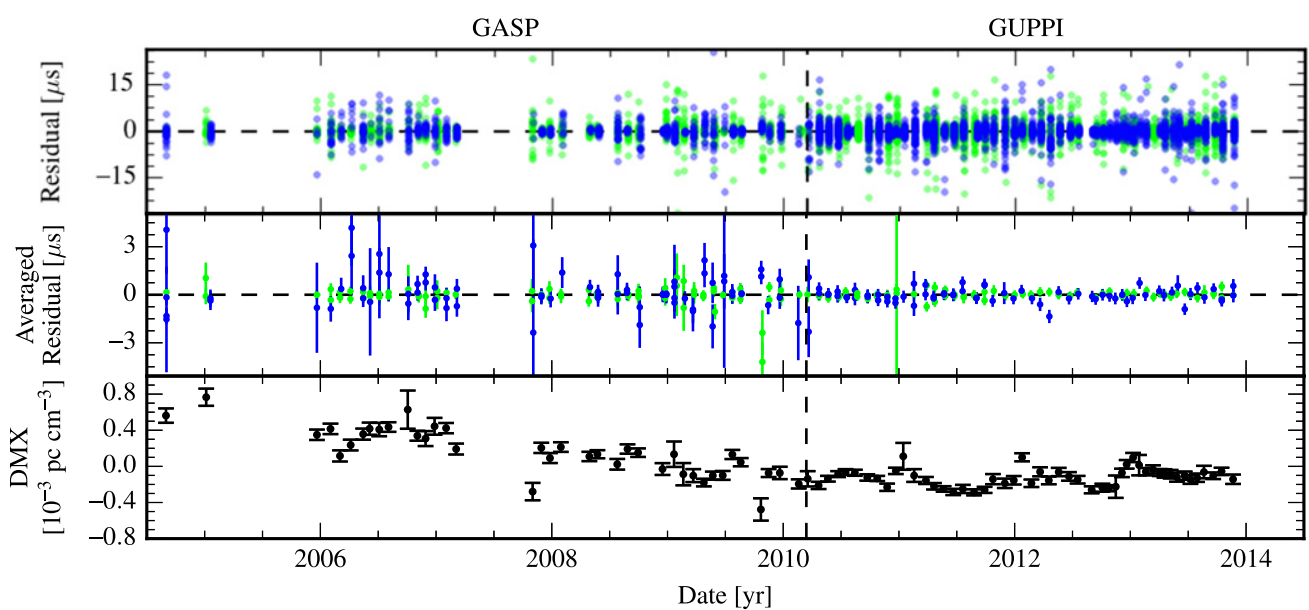

Figure 23. Timing summary for PSR J1744-1134. Colors are blue: $1.4 \mathrm{GHz}$, purple: $2.3 \mathrm{GHz}$, green: $820 \mathrm{MHz}$, orange: $430 \mathrm{MHz}$, red: $327 \mathrm{MHz}$. In the top panel, individual points are semi-transparent; darker regions arise from the overlap of many points.

J1747-4036

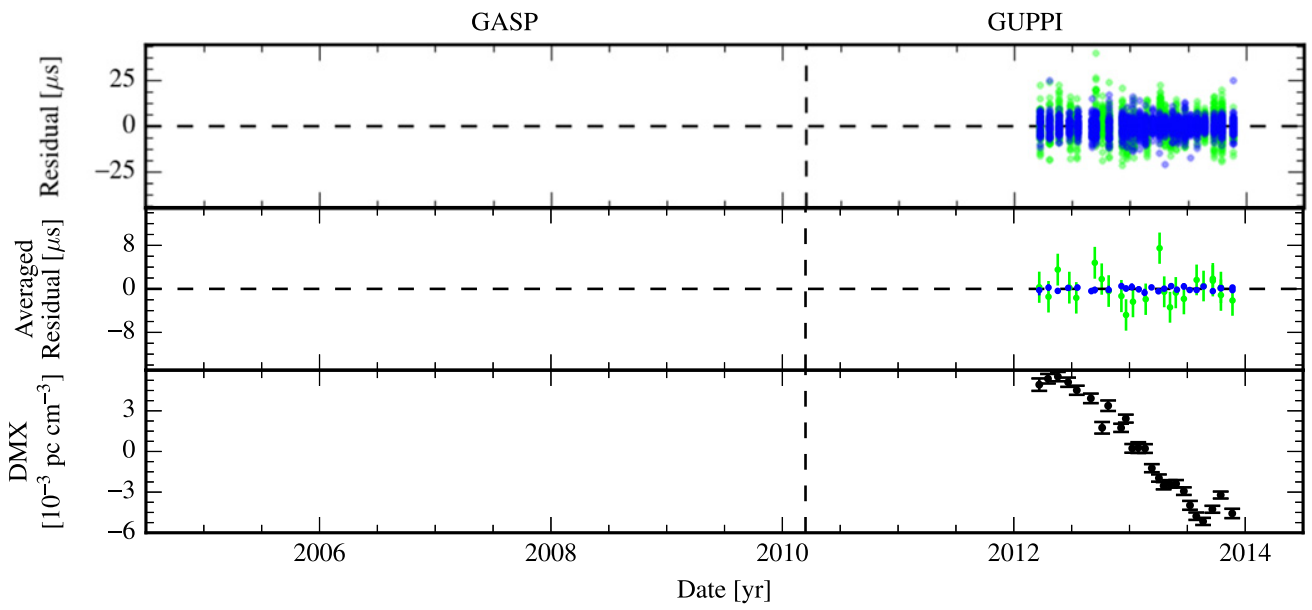

Figure 24. Timing summary for PSR J1747-4036. Colors are blue: $1.4 \mathrm{GHz}$, purple: $2.3 \mathrm{GHz}$, green: $820 \mathrm{MHz}$, orange: $430 \mathrm{MHz}$, red: $327 \mathrm{MHz}$. In the top panel, individual points are semi-transparent; darker regions arise from the overlap of many points.

J1832-0836

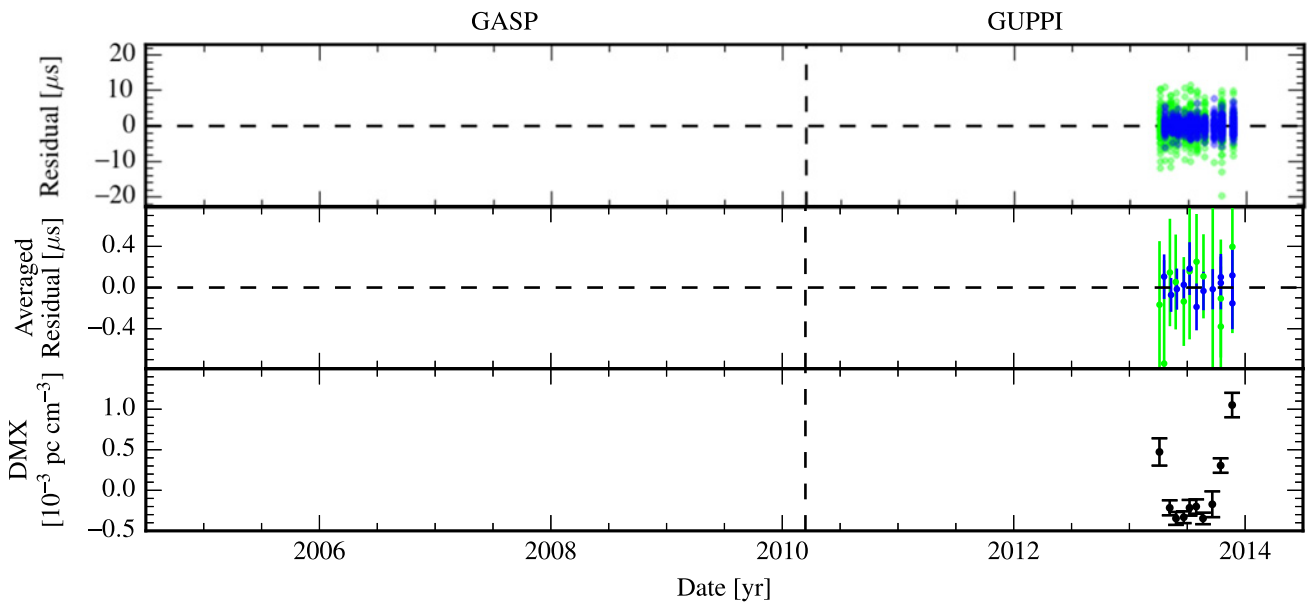

Figure 25. Timing summary for PSR J1832-0836. Colors are blue: $1.4 \mathrm{GHz}$, purple: $2.3 \mathrm{GHz}$, green: $820 \mathrm{MHz}$, orange: $430 \mathrm{MHz}$, red: $327 \mathrm{MHz}$. In the top panel, individual points are semi-transparent; darker regions arise from the overlap of many points. 


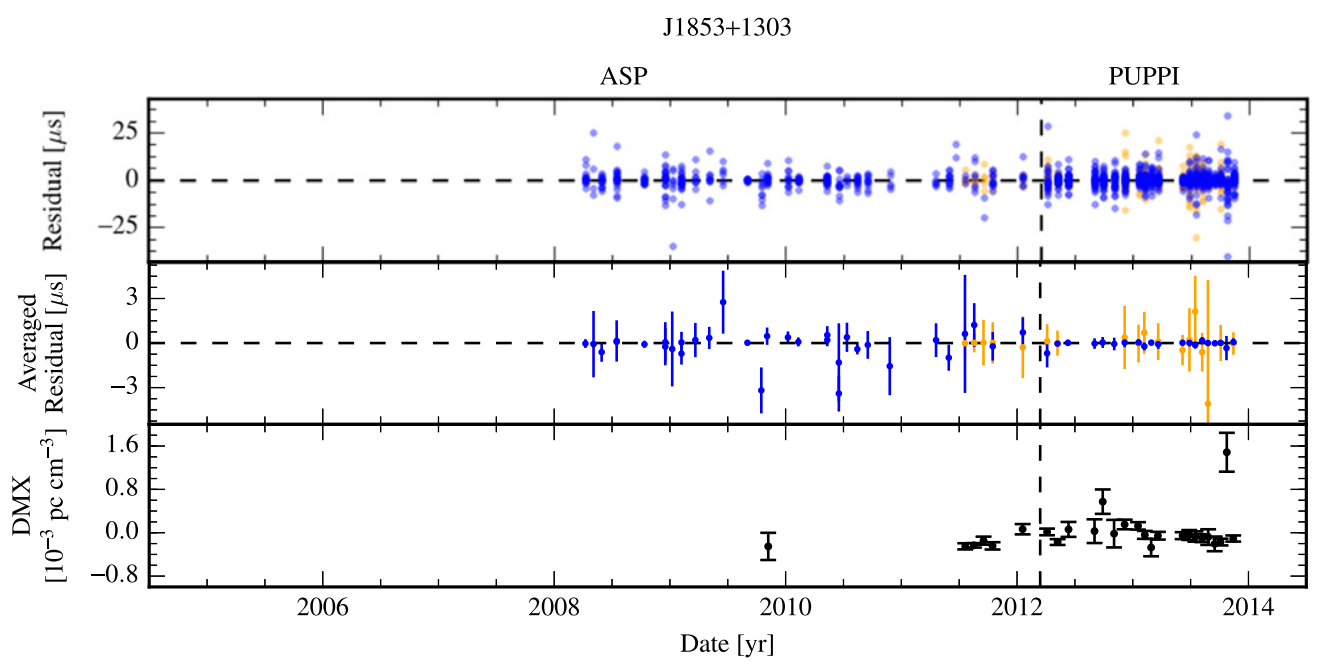

Figure 26. Timing summary for PSR J1853+1303. Colors are blue: $1.4 \mathrm{GHz}$, purple: $2.3 \mathrm{GHz}$, green: $820 \mathrm{MHz}$, orange: $430 \mathrm{MHz}$, red: $327 \mathrm{MHz}$. In the top panel, individual points are semi-transparent; darker regions arise from the overlap of many points.

B1855+09

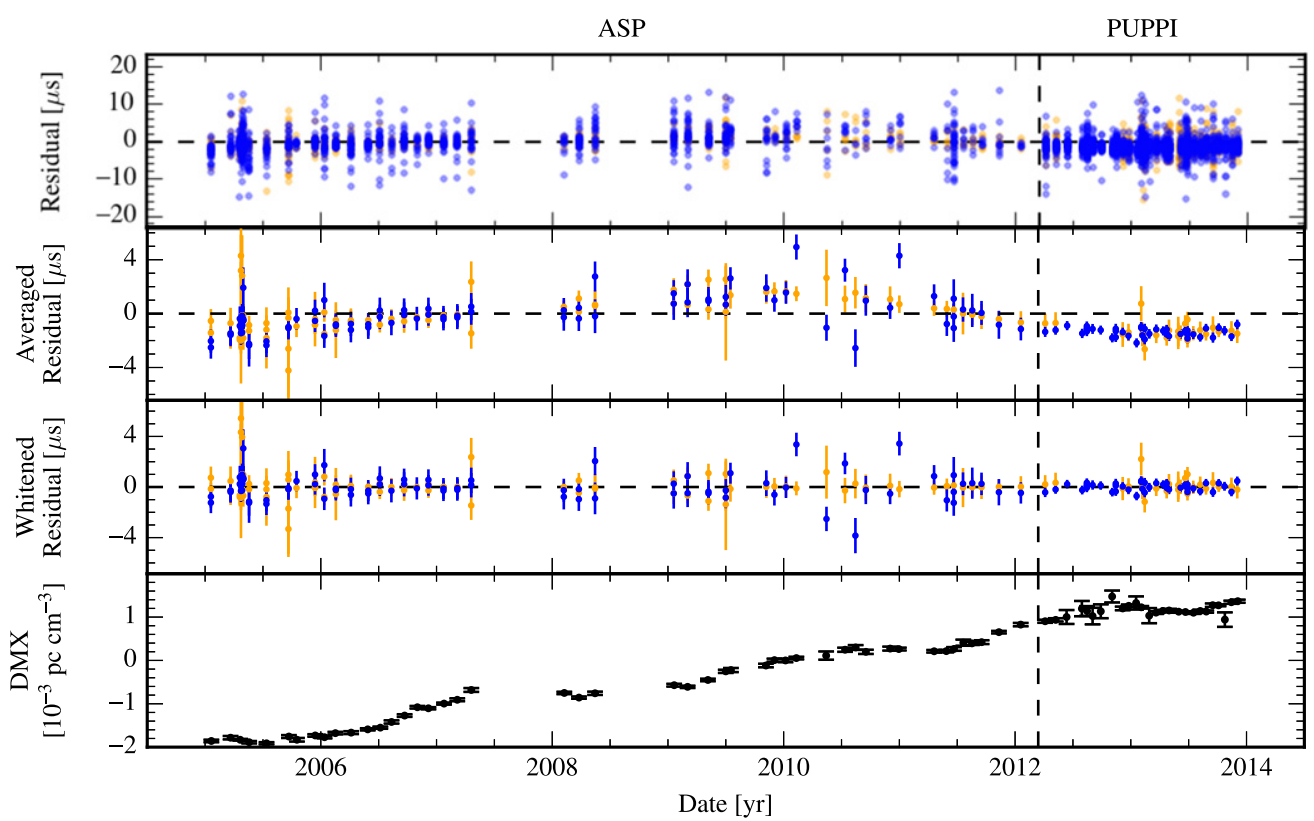

Figure 27. Timing summary for PSR B1855+09. Colors are blue: $1.4 \mathrm{GHz}$, purple: $2.3 \mathrm{GHz}$, green: $820 \mathrm{MHz}$, orange: $430 \mathrm{MHz}$, red: $327 \mathrm{MHz}$. In the top panel, individual points are semi-transparent; darker regions arise from the overlap of many points.

nuisance parameters that we wish to marginalize over. This can be done in a sequential fashion as was presented in Arzoumanian et al. (2014), but here we take a different approach. Notice that all timing parameters are linear and can be described with Gaussian prior distributions. ${ }^{37} \mathrm{We}$ can thus define a combined operator matrix and amplitude vector

$$
T=\left[\begin{array}{lll}
M & F & U
\end{array}\right], \quad \boldsymbol{b}=\left[\begin{array}{c}
\boldsymbol{a} \\
\boldsymbol{j}
\end{array}\right]
$$

\footnotetext{
37 We use uniform priors on the timing model parameter offsets, $\epsilon$ but this is the same as a Gaussian prior with infinite variance. Technically this prior is not normalizable, but since we are interested in parameter estimation and not Bayesian model selection here, this non-normalizable prior is not a problem.
}

with prior distribution

$$
p(\boldsymbol{b} \mid \phi)=\frac{\exp \left(-\frac{1}{2} \boldsymbol{b}^{T} B^{-1} \boldsymbol{b}\right)}{\sqrt{\operatorname{det}(2 \pi B)}}
$$

and covariance matrix defined in terms of the block matrix

$$
B=\left[\begin{array}{ccc}
\infty & 0 & 0 \\
0 & \varphi & 0 \\
0 & 0 & \mathcal{J}
\end{array}\right],
$$




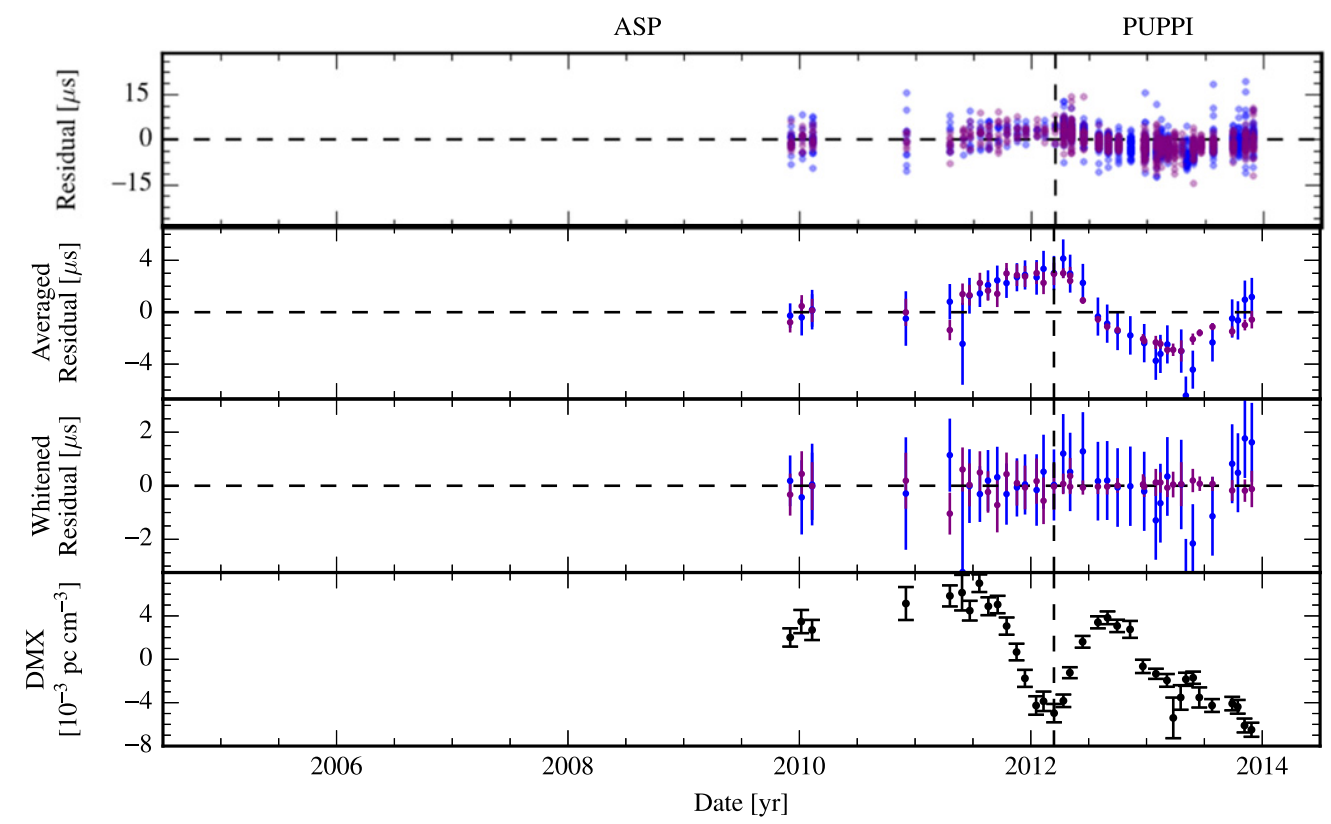

Figure 28. Timing summary for PSR J1903+0327. Colors are blue: $1.4 \mathrm{GHz}$, purple: $2.3 \mathrm{GHz}$, green: $820 \mathrm{MHz}$, orange: $430 \mathrm{MHz}$, red: $327 \mathrm{MHz}$. In the top panel, individual points are semi-transparent; darker regions arise from the overlap of many points.

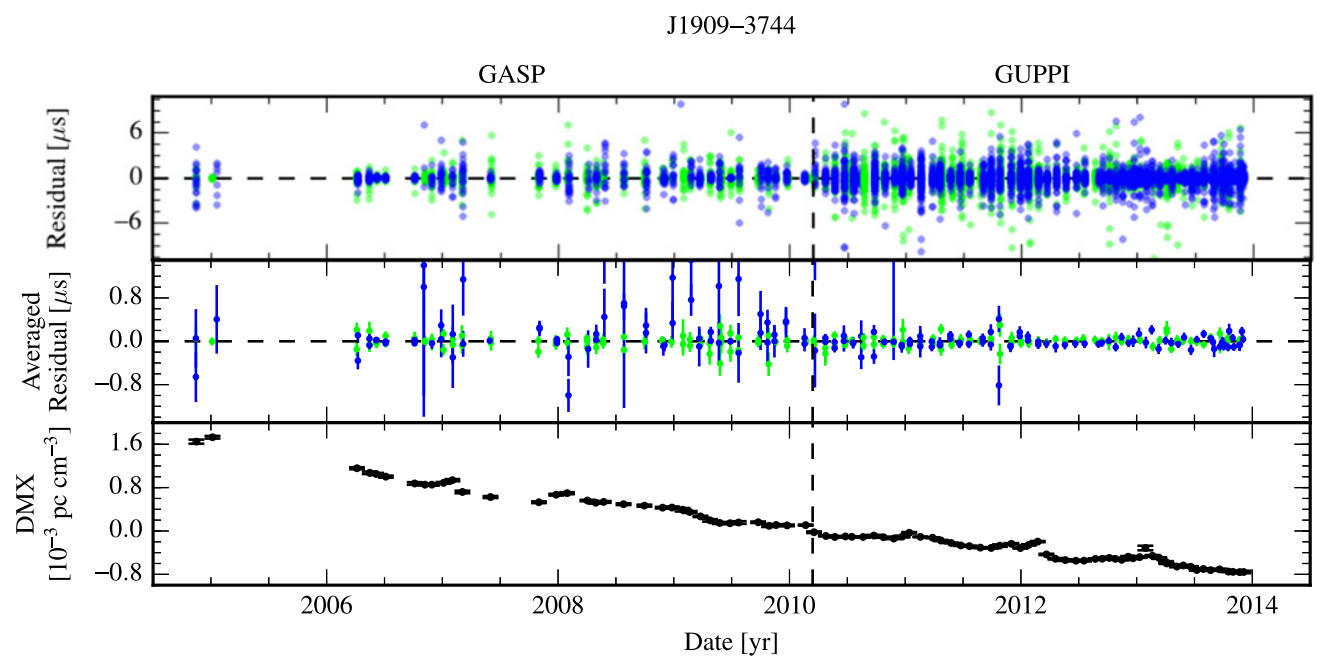

Figure 29. Timing summary for PSR J1909-3744. Colors are blue: $1.4 \mathrm{GHz}$, purple: $2.3 \mathrm{GHz}$, green: $820 \mathrm{MHz}$, orange: $430 \mathrm{MHz}$, red: $327 \mathrm{MHz}$. In the top panel, individual points are semi-transparent; darker regions arise from the overlap of many points.

where $\infty$ is a diagonal matrix of infinities to describe a uniform prior on $\epsilon$. The resulting likelihood function is then

$$
p(\delta \boldsymbol{t} \mid \boldsymbol{b})=\frac{\exp \left[-\frac{1}{2}(\delta \boldsymbol{t}-T \boldsymbol{b})^{T} N^{-1}(\delta \boldsymbol{t}-T \boldsymbol{b})\right]}{\sqrt{\operatorname{det}(2 \pi N)}} .
$$

The marginalized posterior distribution is then

$$
\begin{aligned}
& p(\phi \mid \delta \boldsymbol{t}) \propto \int_{-\infty}^{\infty} d \boldsymbol{\epsilon} d \boldsymbol{a} d \boldsymbol{j} p(\boldsymbol{\epsilon}, \boldsymbol{a}, \boldsymbol{j}, \phi \mid \delta \boldsymbol{t}) \\
& =\int_{-\infty}^{\infty} d \boldsymbol{b} p(\delta \boldsymbol{t} \mid \boldsymbol{b}) p(\boldsymbol{b} \mid \phi) p(\phi) \\
& =\frac{\exp \left[-\frac{1}{2}\left(\delta \boldsymbol{t}^{T} N^{-1} \delta \boldsymbol{t}-\boldsymbol{d}^{T} \Sigma^{-1} \boldsymbol{d}\right)\right]}{\sqrt{(2 \pi)^{N_{\mathrm{TOA}}-\operatorname{dim} \boldsymbol{b}} \operatorname{det}(N) \operatorname{det}(B) \operatorname{det}(\Sigma)}},
\end{aligned}
$$

where

$$
\begin{gathered}
\boldsymbol{d}=T^{T} N^{-1} \delta \boldsymbol{t} \\
\Sigma=\left(B^{-1}+T^{T} N^{-1} T\right) .
\end{gathered}
$$

The maximum likelihood values of $\boldsymbol{b}$ and their uncertainties can be found as

$$
\begin{gathered}
\hat{\boldsymbol{b}}=\Sigma^{-1} \boldsymbol{d} \\
\operatorname{cov}(\boldsymbol{b})=\Sigma^{-1} .
\end{gathered}
$$

This scheme has the advantage of being computationally efficient in that it bypasses $O\left(N_{\mathrm{TOA}}^{3}\right)$ matrix operations via rank reduced matrices (van Haasteren \& Vallisneri 2015) resulting in a likelihood evaluation that instead scales as $O\left(N_{\text {par }}^{3}\right)$, where $N_{\text {par }}$ is the sum of the number of timing parameters, red noise 


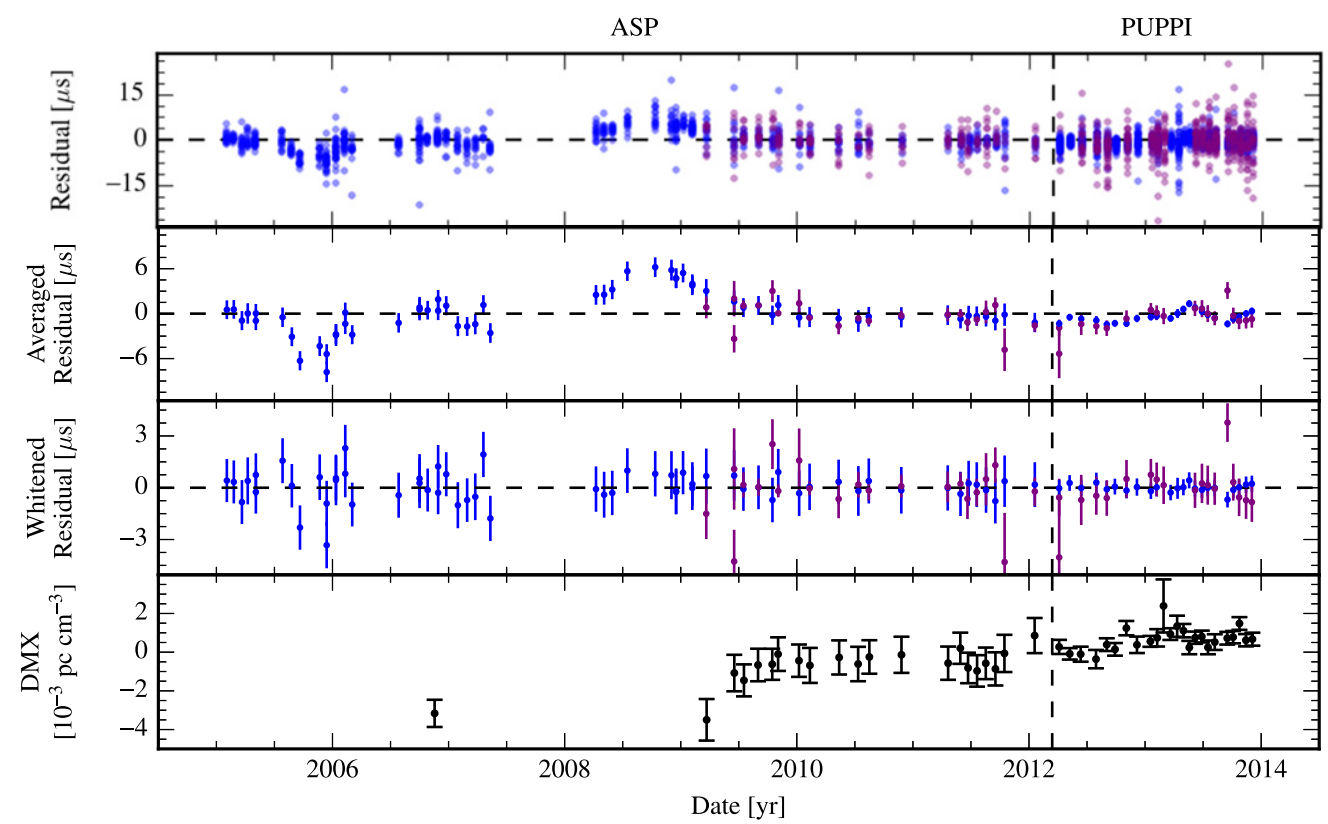

Figure 30. Timing summary for PSR J1910+1256. Colors are blue: $1.4 \mathrm{GHz}$, purple: $2.3 \mathrm{GHz}$, green: $820 \mathrm{MHz}$, orange: $430 \mathrm{MHz}$, red: $327 \mathrm{MHz}$. In the top panel, individual points are semi-transparent; darker regions arise from the overlap of many points.

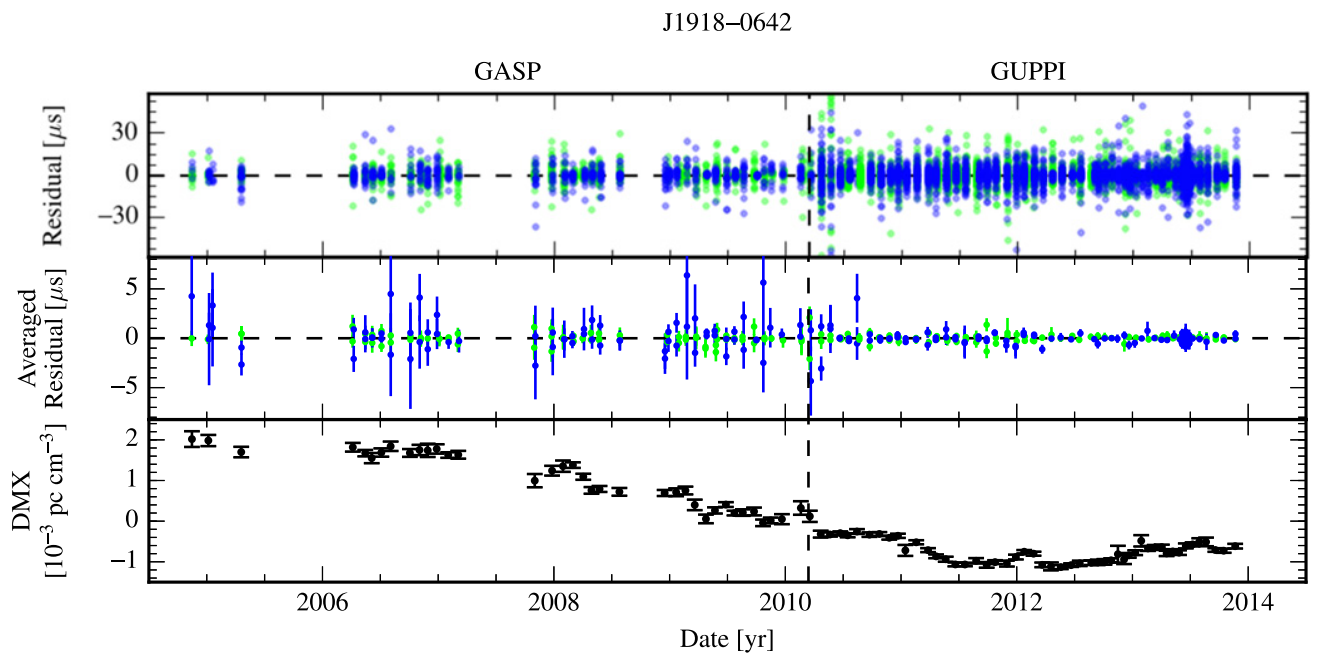

Figure 31. Timing summary for PSR J1918-0642. Colors are blue: $1.4 \mathrm{GHz}$, purple: $2.3 \mathrm{GHz}$, green: $820 \mathrm{MHz}$, orange: $430 \mathrm{MHz}$, red: $327 \mathrm{MHz}$. In the top panel, individual points are semi-transparent; darker regions arise from the overlap of many points.

sample frequencies, and observing time bins. For the largest datasets the computational speed up is a factor of $\sim 10^{3}$.

For a given set of hyper-parameters, this allows us to determine the maximum likelihood timing model parameters and the maximum likelihood red noise realization present in the data via the equivalent of a generalized least squares fit. We can also evaluate the posterior of the hyper-parameters $\phi$ and thus find the maximum likelihood noise parameters including the EFAC, EQUAD, ECORR, red noise amplitude $A_{\text {red, }}$, and spectral index $\gamma_{\text {red }}$. The posterior distributions of the noise parameters are sampled using a Markov Chain Monte Carlo process in which we sample some parameters in $\log _{10}$ space and limit them to $\log _{10} J_{k} \in[-8.5,-4]$, with $J_{k}$ in units of seconds, $\log _{10} A_{\text {red }} \in[-7.5,1.5]$ where $A_{\text {red }}$ is in units of $\mu \mathrm{s}$ $\mathrm{yr}^{1 / 2}$, and $\gamma_{\text {red }} \in[0,7]$.

\section{APPENDIX D \\ DAILY AVERAGED RESIDUALS}

For modern wide-band timing campaigns using multi-channel TOAs it becomes useful to visually inspect timing residuals that have been averaged in order to look for long term trends or biases. Here we derive a robust weighted average that will fully account for short timescale correlations introduced by the ECORR in our noise models. This is important since ECORR is meant to model pulse phase jitter, thus when constructing 


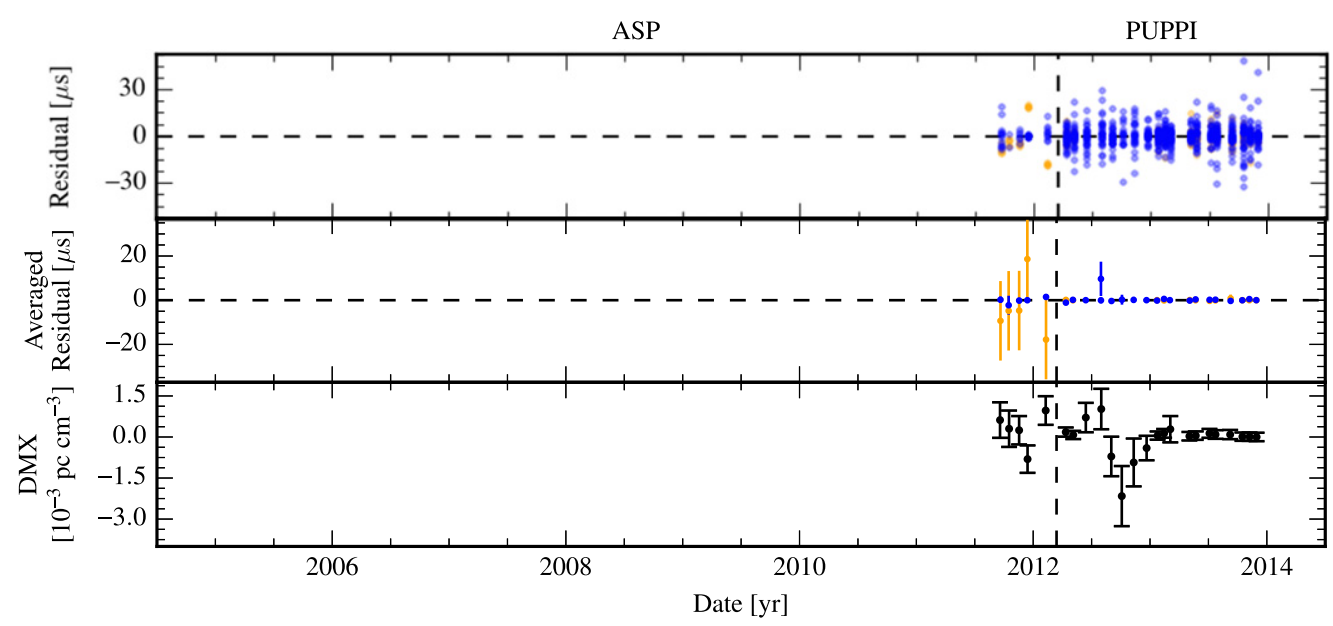

Figure 32. Timing summary for PSR J1923+2515. Colors are blue: $1.4 \mathrm{GHz}$, purple: $2.3 \mathrm{GHz}$, green: $820 \mathrm{MHz}$, orange: $430 \mathrm{MHz}$, red: $327 \mathrm{MHz}$. In the top panel, individual points are semi-transparent; darker regions arise from the overlap of many points.

B1937+21

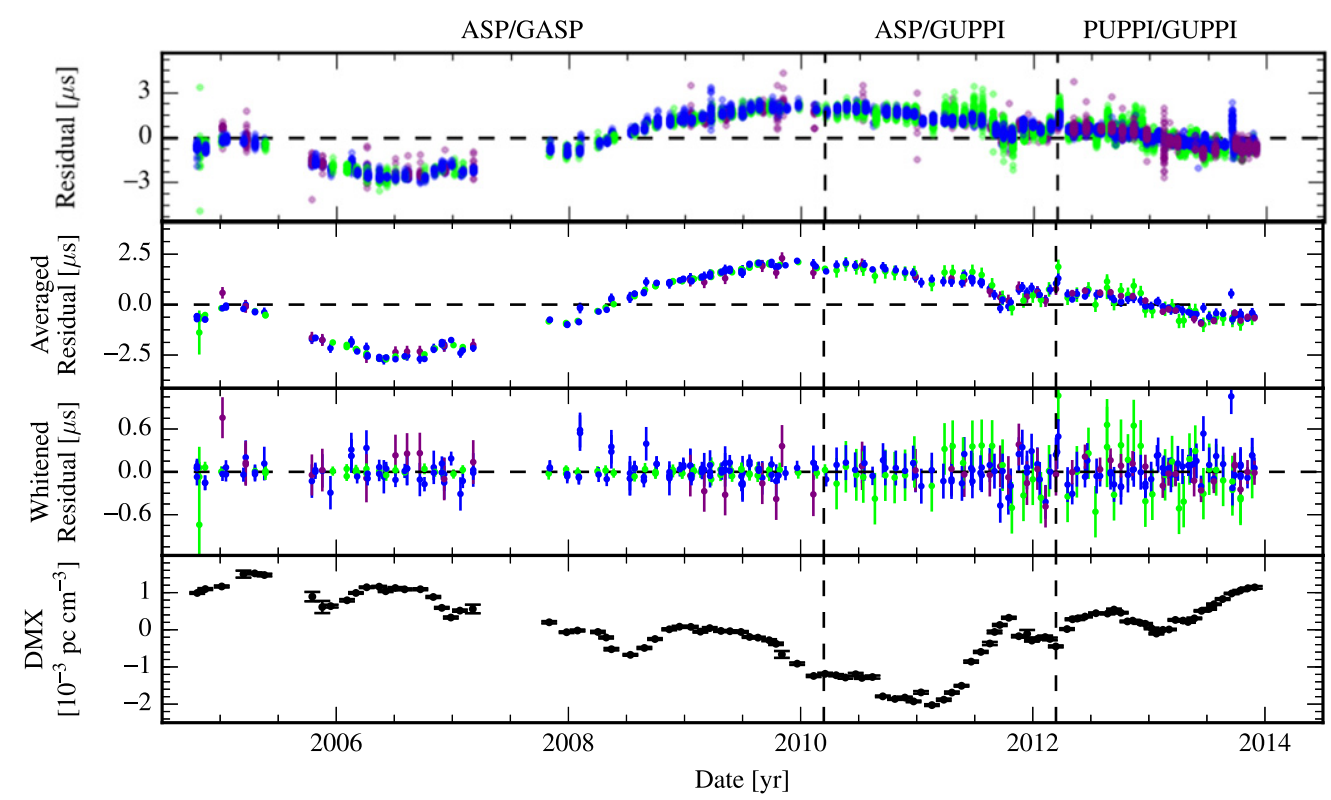

Figure 33. Timing summary for PSR B1937+21. Colors are blue: $1.4 \mathrm{GHz}$, purple: $2.3 \mathrm{GHz}$, green: $820 \mathrm{MHz}$, orange: $430 \mathrm{MHz}$, red: $327 \mathrm{MHz}$. In the top panel, individual points are semi-transparent; darker regions arise from the overlap of many points.

daily averaged residuals, one must include this effect as it results in larger averaged uncertainties on the averaged residuals. In essence this allows for a way to visually determine which pulsars may be dominated by pulse phase jitter.

We begin the derivation by introducing the probability distribution of the group of residuals that belong to time $\operatorname{bin}^{38} k$,

$$
\begin{aligned}
& p\left(\delta \boldsymbol{t}_{k} \mid \bar{\delta} t_{k}\right) \\
& =\frac{\exp \left[-\frac{1}{2}\left(\delta \boldsymbol{t}_{k}-O \bar{\delta} t_{k}\right)^{T} C_{k}^{-1}\left(\delta \boldsymbol{t}_{k}-O \bar{\delta} t_{k}\right)\right]}{\operatorname{det}\left(C_{k}\right)},
\end{aligned}
$$

\footnotetext{
$\overline{38}$ In this work, we have used time bins of size $1 \mathrm{~s}$, thus are only averaging sets of multi-channel residuals measured simultaneously.
}

where $\delta t_{k}, \bar{\delta} t_{k}$, and $C_{k}$ are the residuals in time bin $k$, the mean residual in time bin $k$, and the covariance matrix of the residuals in time bin $k$, respectively. Here, $O$ is the design matrix for the mean which in this case is a vector of ones of length $N_{k}$, where $N_{k}$ is the number of residuals in simultaneous time bin $k$. In an identical manner as Appendix $\mathrm{C}$ we can determine the maximum likelihood estimator and uncertainty for the mean of the probability distribution function (i.e., the daily averaged residual)

$$
\begin{gathered}
\bar{\delta} t_{k}^{\mathrm{ML}}=\left(O^{T} C_{k}^{-1} O\right)^{-1} O^{T} C_{k}^{-1} \delta t_{k} \\
\bar{\sigma}_{k}^{2}=\left(O^{T} C_{k}^{-1} O\right)^{-1}
\end{gathered}
$$




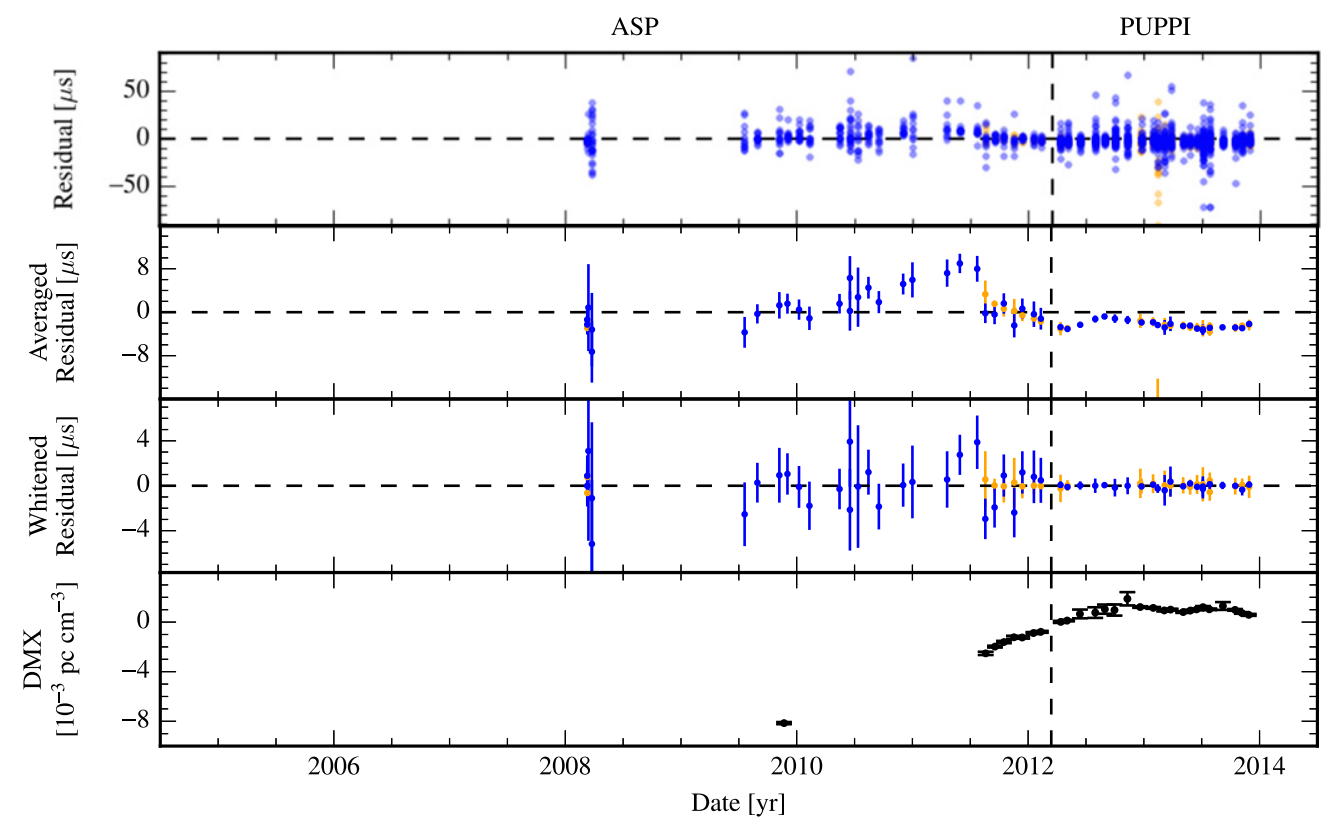

Figure 34. Timing summary for PSR J1944+0907. Colors are blue: $1.4 \mathrm{GHz}$, purple: $2.3 \mathrm{GHz}$, green: $820 \mathrm{MHz}$, orange: $430 \mathrm{MHz}$, red: $327 \mathrm{MHz}$. In the top panel, individual points are semi-transparent; darker regions arise from the overlap of many points.

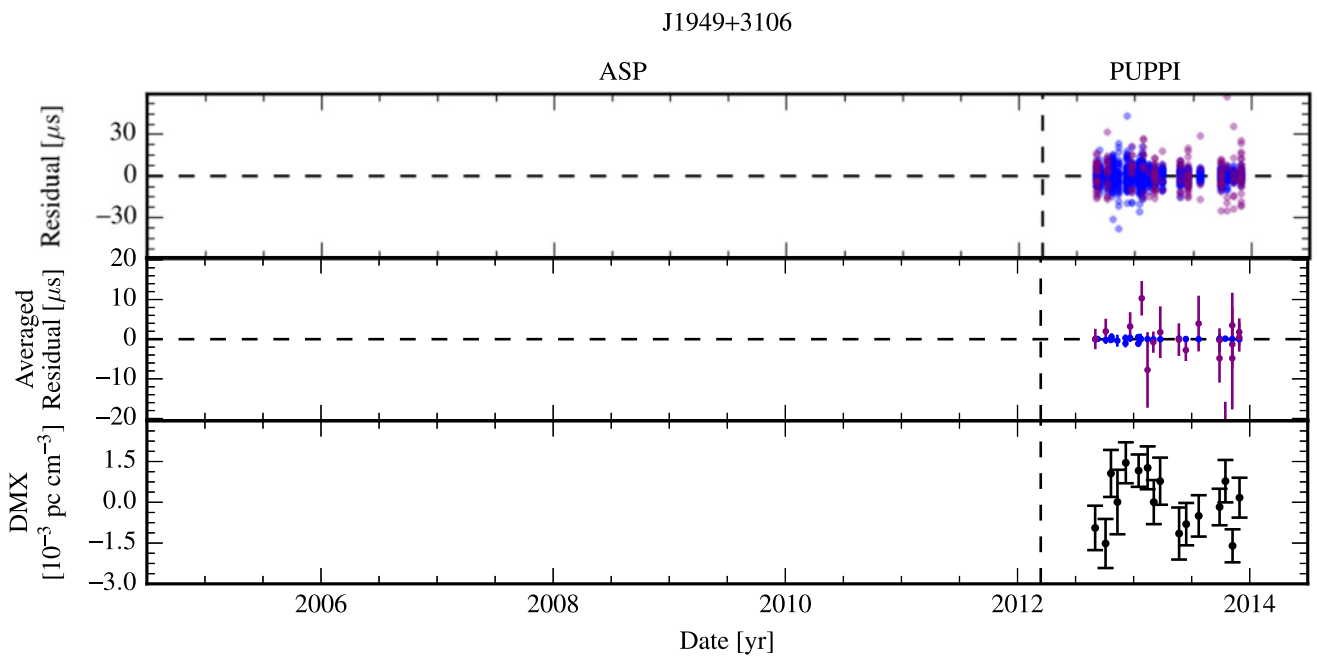

Figure 35. Timing summary for PSR J1949+3106. Colors are blue: $1.4 \mathrm{GHz}$, purple: $2.3 \mathrm{GHz}$, green: $820 \mathrm{MHz}$, orange: $430 \mathrm{MHz}$, red: $327 \mathrm{MHz}$. In the top panel, individual points are semi-transparent; darker regions arise from the overlap of many points.

where $\bar{\sigma}_{k}$ is the weighted uncertainty on the daily averaged residual. Note that if $C_{k}$ is diagonal with elements corresponding to the TOA uncertainties then we obtain our usual expression for the weighted mean and standard deviation

$$
\bar{\delta} t_{k}^{\mathrm{ML}}=\frac{\sum_{i=1}^{N_{k}} \delta \boldsymbol{t}_{i, k} \sigma_{i, k}^{-2}}{\sum_{i=1}^{N_{k}} \sigma_{i, k}^{-2}}
$$

$$
\bar{\sigma}_{k}^{2}=\left(\sum_{i=1}^{N_{k}} \sigma_{i, k}^{-2}\right)^{-1},
$$

where $\sigma_{i, k}$ is the TOA uncertainty for the $i$ TOA in simultaneous time bin $k$. We note that the ECORR will add to the off-diagonal components of $C_{k}$ and can have a large impact depending on the relative strength of ECORR compared to the radiometer noise component. 


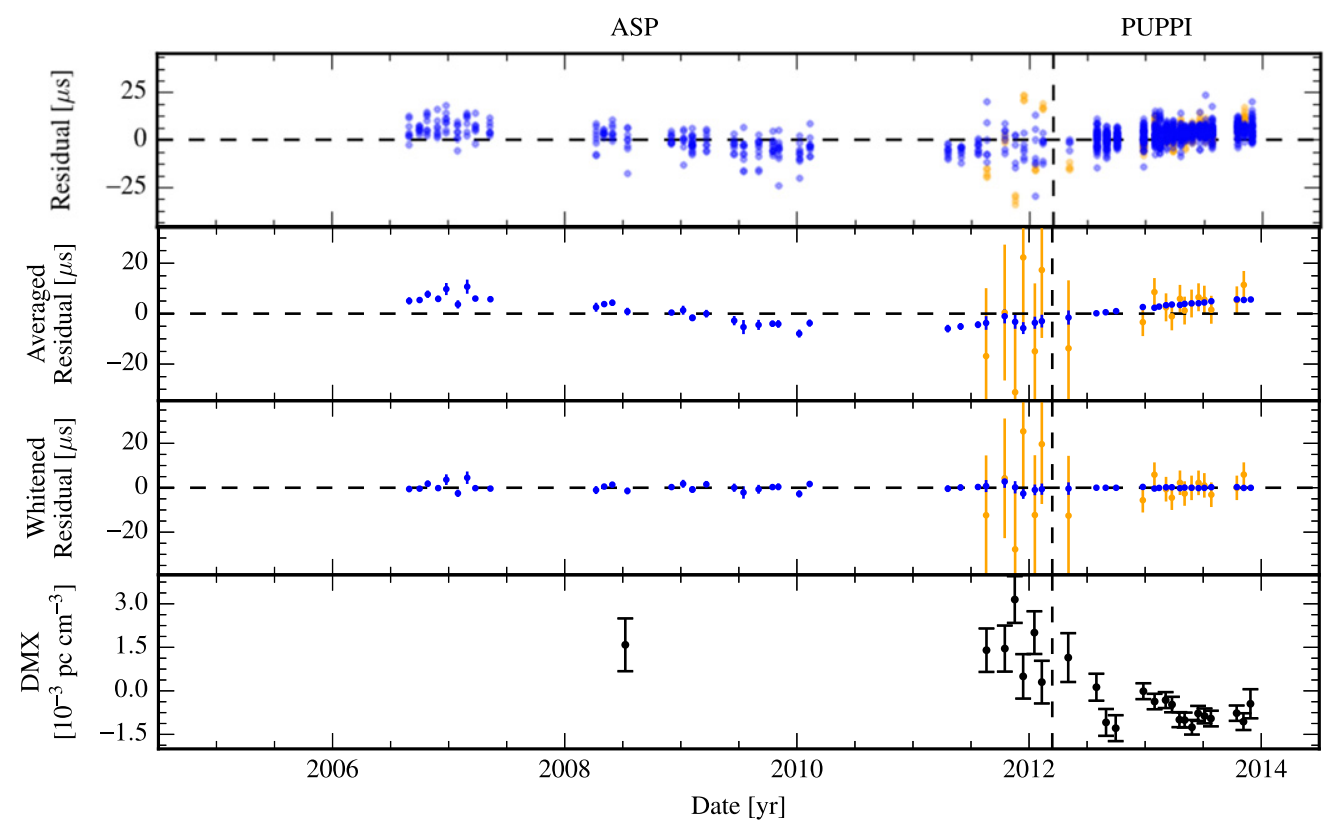

Figure 36. Timing summary for PSR B1953+29. Colors are blue: $1.4 \mathrm{GHz}$, purple: $2.3 \mathrm{GHz}$, green: $820 \mathrm{MHz}$, orange: $430 \mathrm{MHz}$, red: $327 \mathrm{MHz}$. In the top panel, individual points are semi-transparent; darker regions arise from the overlap of many points.

J2010-1323

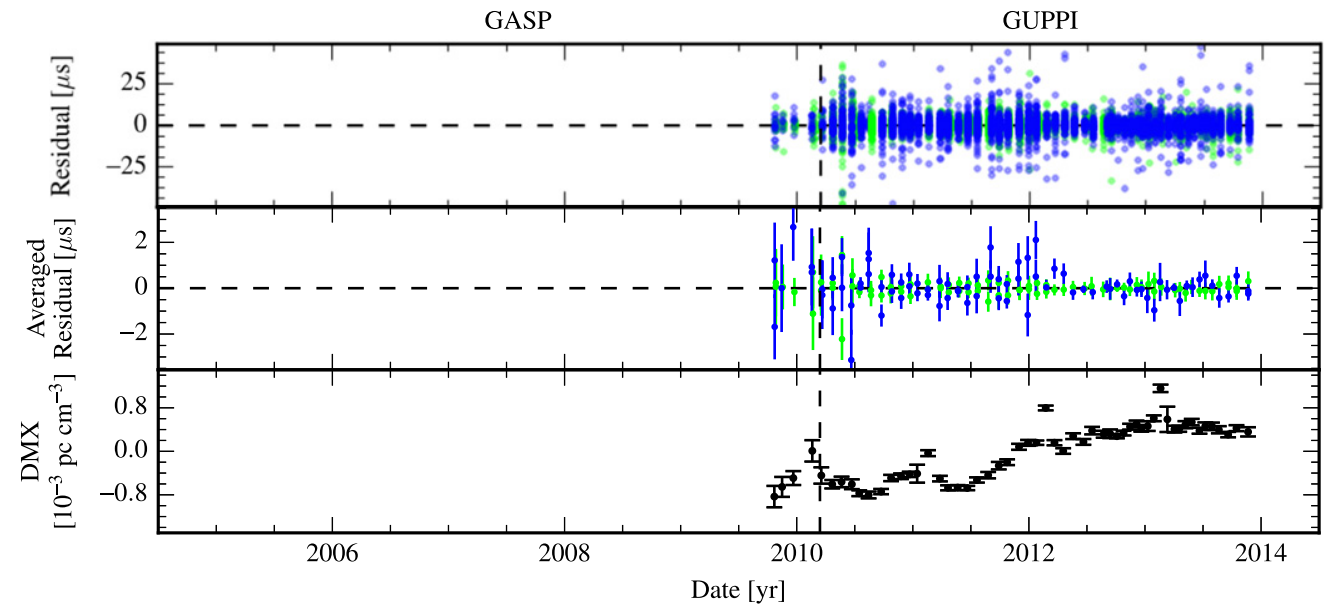

Figure 37. Timing summary for PSR J2010-1323. Colors are blue: $1.4 \mathrm{GHz}$, purple: $2.3 \mathrm{GHz}$, green: $820 \mathrm{MHz}$, orange: $430 \mathrm{MHz}$, red: $327 \mathrm{MHz}$. In the top panel, individual points are semi-transparent; darker regions arise from the overlap of many points. 


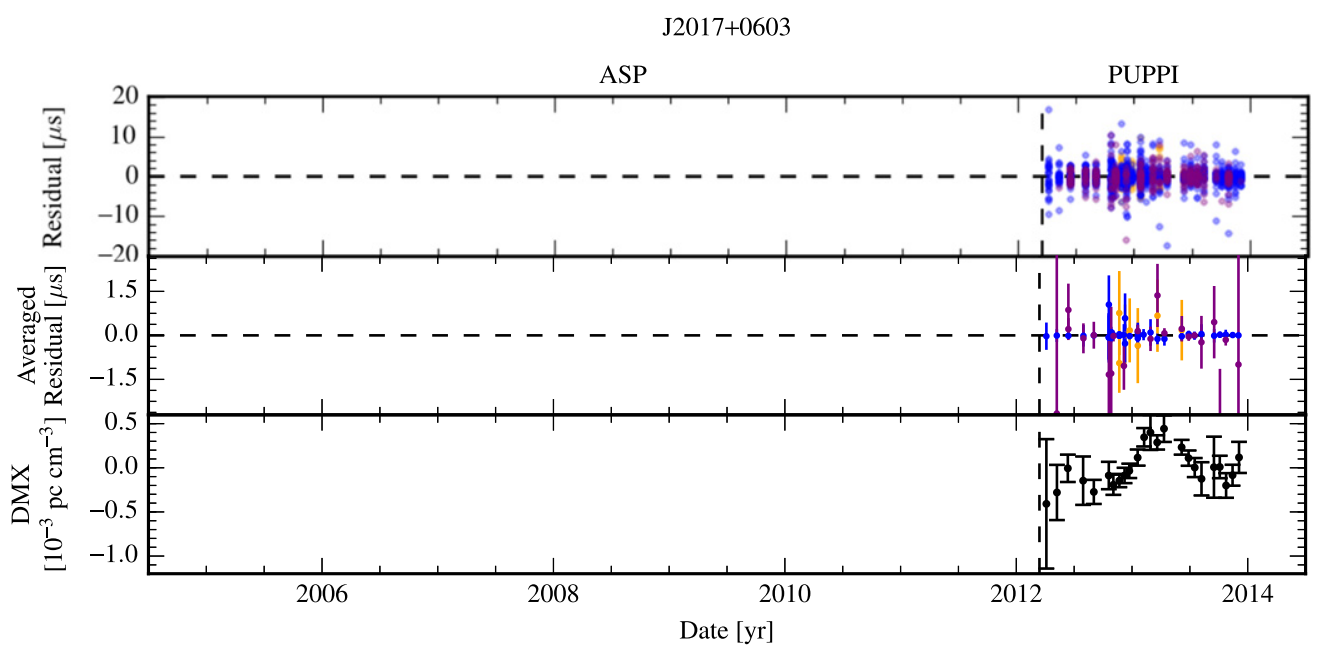

Figure 38. Timing summary for PSR J2017+0603. Colors are blue: $1.4 \mathrm{GHz}$, purple: $2.3 \mathrm{GHz}$, green: $820 \mathrm{MHz}$, orange: $430 \mathrm{MHz}$, red: $327 \mathrm{MHz}$. In the top panel, individual points are semi-transparent; darker regions arise from the overlap of many points.

$\mathrm{J} 2043+1711$

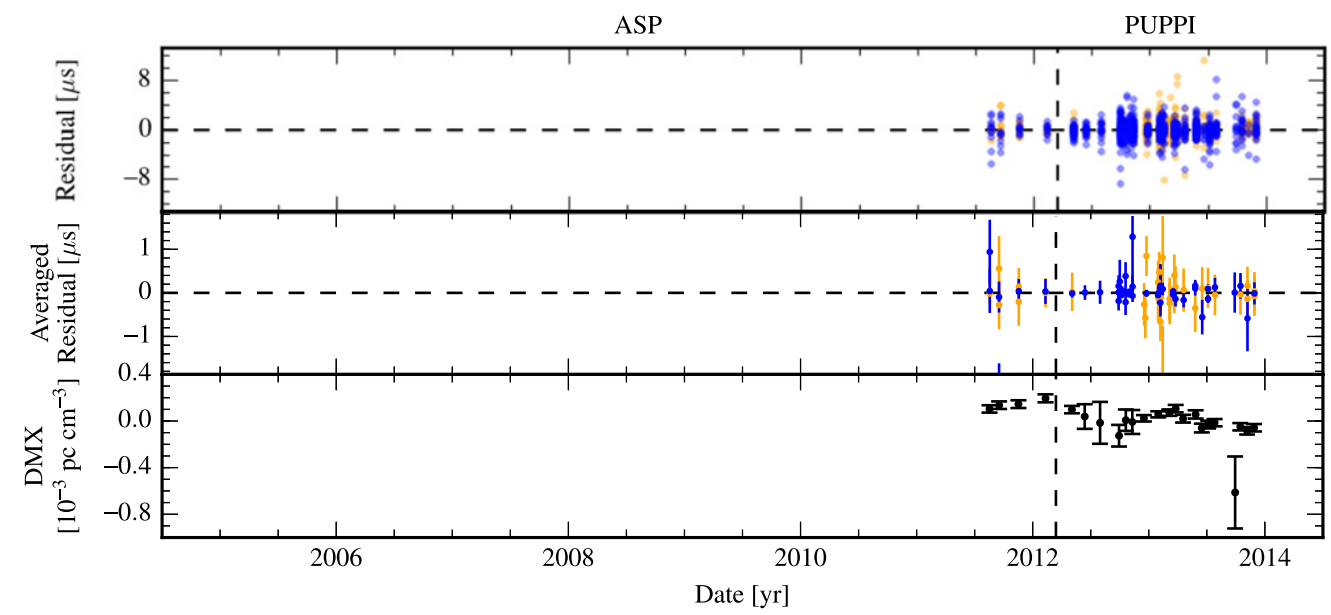

Figure 39. Timing summary for PSR J2043+1711. Colors are blue: $1.4 \mathrm{GHz}$, purple: $2.3 \mathrm{GHz}$, green: $820 \mathrm{MHz}$, orange: $430 \mathrm{MHz}$, red: $327 \mathrm{MHz}$. In the top panel, individual points are semi-transparent; darker regions arise from the overlap of many points.

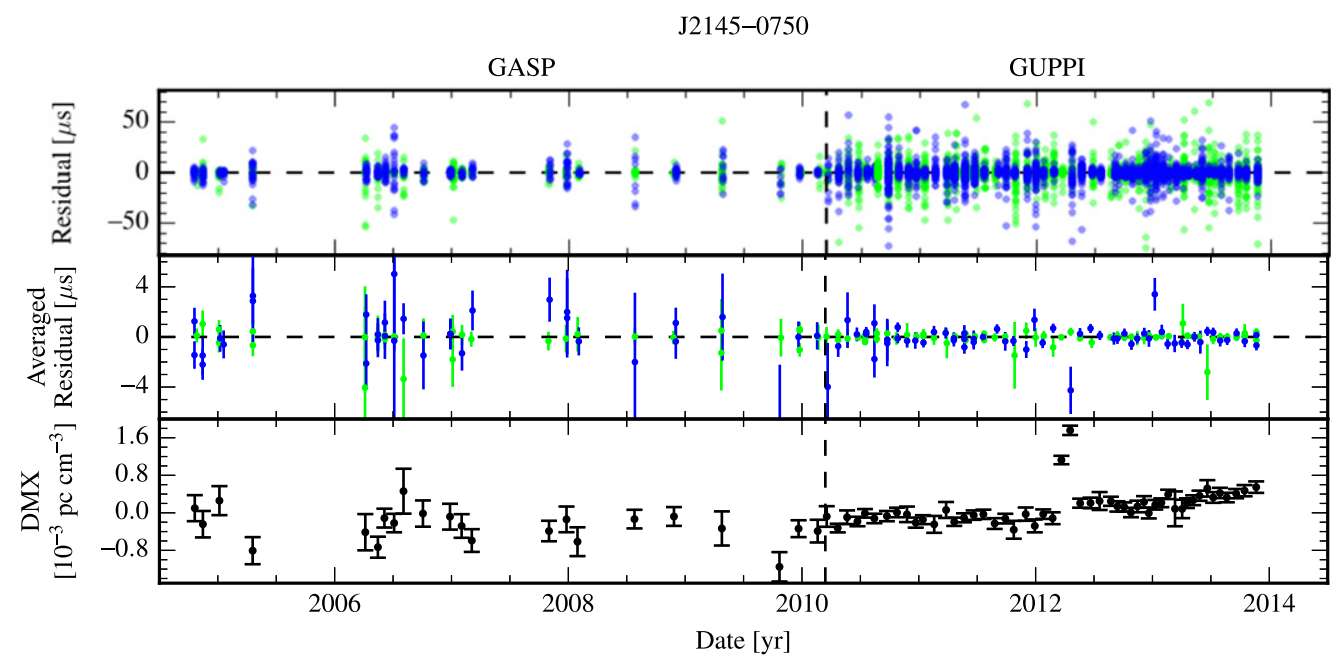

Figure 40. Timing summary for PSR J2145-0750. Colors are blue: $1.4 \mathrm{GHz}$, purple: $2.3 \mathrm{GHz}$, green: $820 \mathrm{MHz}$, orange: $430 \mathrm{MHz}$, red: $327 \mathrm{MHz}$. In the top panel, individual points are semi-transparent; darker regions arise from the overlap of many points. 


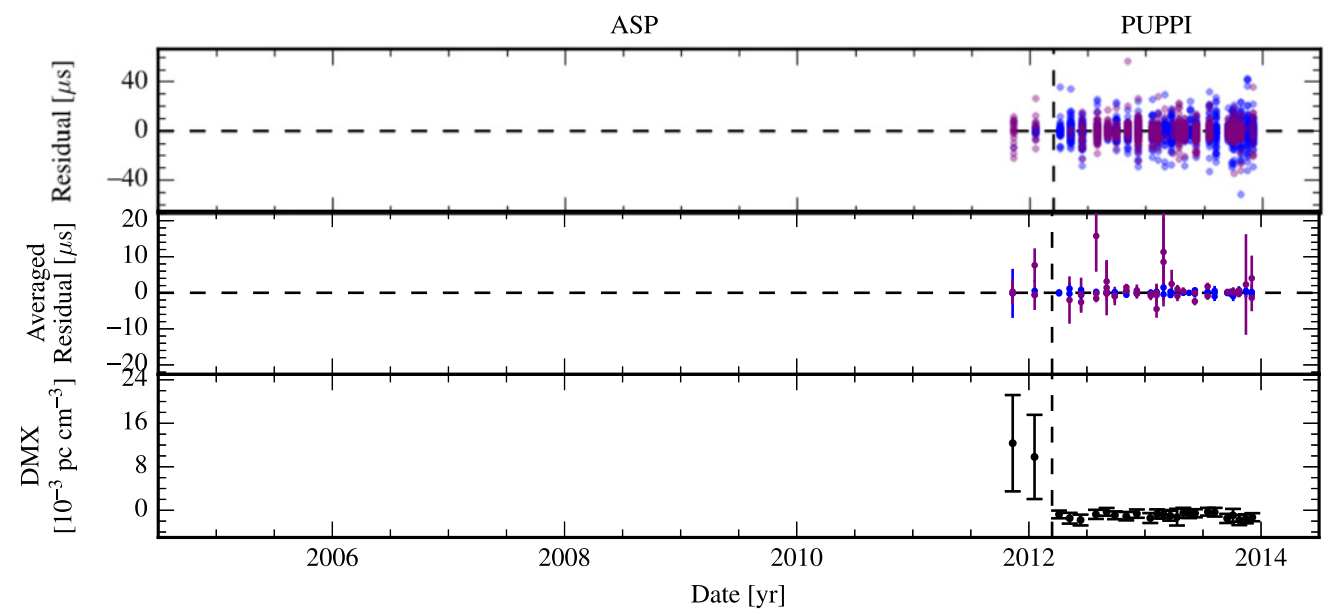

Figure 41. Timing summary for PSR J2214+3000. Colors are blue: $1.4 \mathrm{GHz}$, purple: $2.3 \mathrm{GHz}$, green: $820 \mathrm{MHz}$, orange: $430 \mathrm{MHz}$, red: $327 \mathrm{MHz}$. In the top panel, individual points are semi-transparent; darker regions arise from the overlap of many points.

$\mathrm{J} 2302+4442$

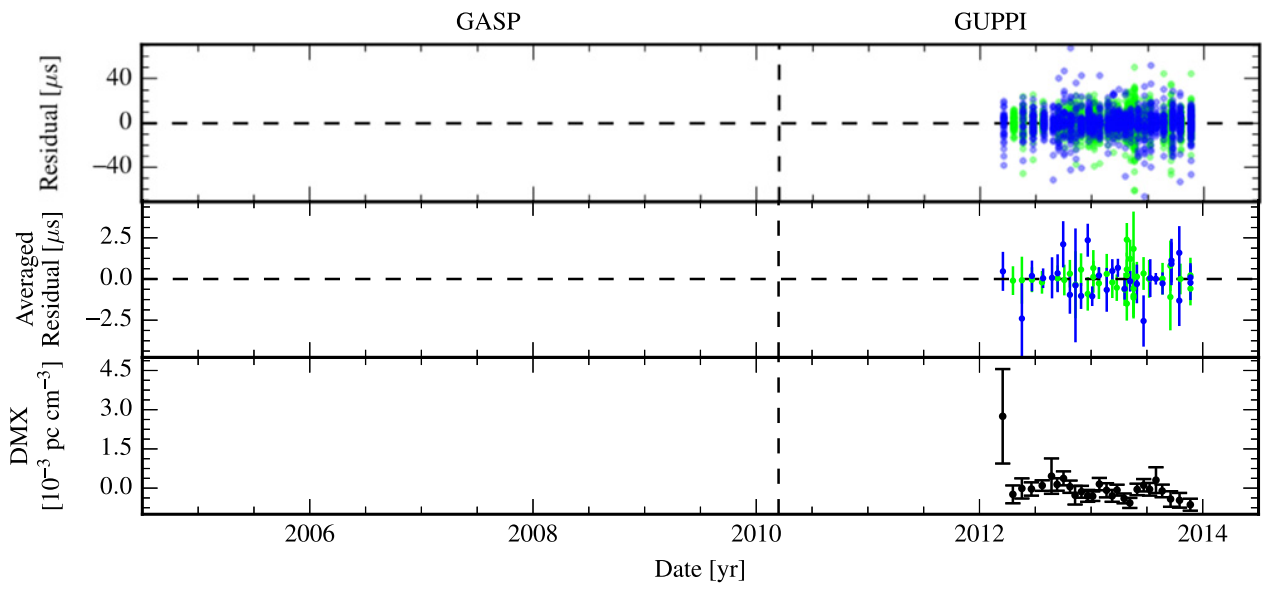

Figure 42. Timing summary for PSR J2302+4442. Colors are blue: $1.4 \mathrm{GHz}$, purple: $2.3 \mathrm{GHz}$, green: $820 \mathrm{MHz}$, orange: $430 \mathrm{MHz}$, red: $327 \mathrm{MHz}$. In the top panel, individual points are semi-transparent; darker regions arise from the overlap of many points.

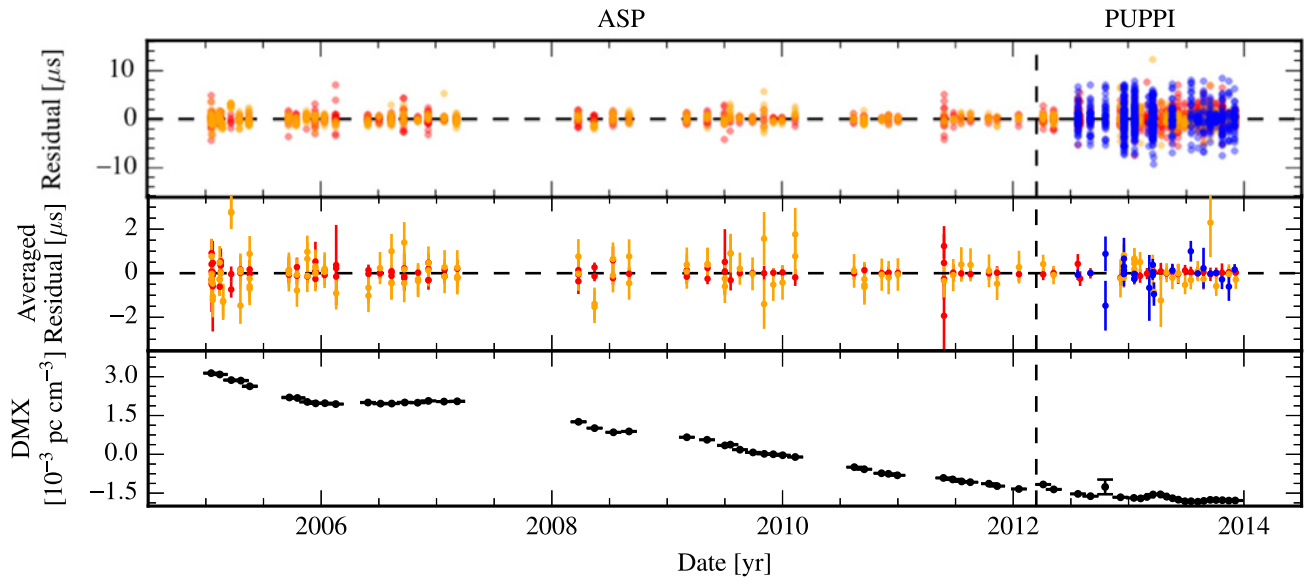

Figure 43. Timing summary for PSR J2317+1439. Colors are blue: $1.4 \mathrm{GHz}$, purple: $2.3 \mathrm{GHz}$, green: $820 \mathrm{MHz}$, orange: $430 \mathrm{MHz}$, red: $327 \mathrm{MHz}$. In the top panel, individual points are semi-transparent; darker regions arise from the overlap of many points. 


\section{REFERENCES}

Arzoumanian, Z., Brazier, A., Burke-Spolaor, S., et al. 2014, ApJ, 794, 141 Arzoumanian, Z., Nice, D. J., Taylor, J. H., \& Thorsett, S. E. 1994, ApJ, 422,671

Blandford, R. D., Narayan, R., \& Romani, R. W. 1984, JApA, 5, 369

Coles, W., Hobbs, G., Champion, D. J., Manchester, R. N., \& Verbiest, J. P. W. 2011, MNRAS, 418, 561

Cordes, J. M., \& Downs, G. S. 1985, ApJS, 59, 343

Cordes, J. M., \& Shannon, R. M. 2010, arXiv:1010.3785

Cordes, J. M., Shannon, R. M., \& Stinebring, D. R. 2015, ApJ, submitted (arXiv:1503.08491)

Damour, T., \& Deruelle, N. 1985, AnIHP, 43, 107

Damour, T., \& Taylor, J. H. 1992, PhRvD, 45, 1840

Demorest, P. B. 2007, PhD thesis, Univ. California

Demorest, P. B., Ferdman, R. D., Gonzalez, M. E., et al. 2013, ApJ, 762, 94 Detweiler, S. 1979, ApJ, 234, 1100

Dolch, T., Lam, M. T., Cordes, J., et al. 2014, ApJ, 794, 21

DuPlain, R., Ransom, S., Demorest, P., et al. 2008, Proc. SPIE, 7019, 70191D Ellis, J. A. 2013, CQGra, 30, 224004

Ellis, J. A. 2014, PhD thesis, Univ. Wisconsin

Feroz, F., Hobson, M. P., \& Bridges, M. 2009, MNRAS, 398, 1601

Fonseca, E., Stairs, I. H., \& Thorsett, S. E. 2014, ApJ, 787, 82

Ford, J. M., Demorest, P., \& Ransom, S. 2010, Proc. SPIE, 7740, 0

Foster, R. S., \& Cordes, J. M. 1990, ApJ, 364, 123

Grishchuk, L. P. 2005, PhyU, 48, 1235

Hellings, R. W., \& Downs, G. S. 1983, ApJ, 265, L39

Hobbs, G., Archibald, A., Arzoumanian, Z., et al. 2010, CQGra, 27, 084013

Hotan, A. W., Bailes, M., \& Ord, S. M. 2005, MNRAS, 362, 1267

Hotan, A. W., van Straten, W., \& Manchester, R. N. 2004, PASA, 21, 302

Jaffe, A. H., \& Backer, D. C. 2003, ApJ, 583, 616

Keith, M. J., Coles, W., Shannon, R. M., et al. 2013, MNRAS, 429, 2161

Kramer, M., Stairs, I. H., Manchester, R. N., et al. 2006, Sci, 314, 97
Lam, M. T., Cordes, J. M., Chatterjee, S., \& Dolch, T. 2015, ApJ, 801, 130 Lange, C., Camilo, F., Wex, N., et al. 2001, MNRAS, 326, 274

Lentati, L., Alexander, P., Hobson, M. P., et al. 2014, MNRAS, 437, 3004

Lentati, L., Taylor, S. R., Mingarelli, C. M. F., et al. 2015, MNRAS, 453, 2576

Lorimer, D. R., \& Kramer, M. 2005, Handbook of Pulsar Astronomy (Cambridge: Cambridge Univ. Press)

Madison, D. R., Cordes, J. M., \& Chatterjee, S. 2014, ApJ, 788, 141

Manchester, R. N., Hobbs, G., Bailes, M., et al. 2013, PASA, 30, 17

McWilliams, S. T., Ostriker, J. P., \& Pretorius, F. 2014, ApJ, 789, 156

Pennucci, T. T., Demorest, P. B., \& Ransom, S. M. 2014, ApJ, 790, 93

Ramachandran, R., Demorest, P., Backer, D. C., Cognard, I., \& Lommen, A 2006, ApJ, 645, 303

Rickett, B. J. 1990, ARA\&A, 28, 561

Sazhin, M. V. 1978, SvA, 22, 36

Sesana, A. 2013, MNRAS, 433, L1

Sesana, A., Vecchio, A., \& Colacino, C. N. 2008, MNRAS, 390, 192

Sesana, A., Vecchio, A., \& Volonteri, M. 2009, MNRAS, 394, 2255

Shannon, R. M., \& Cordes, J. M. 2010, ApJ, 725, 1607

Shannon, R. M., Cordes, J. M., Metcalfe, T. S., et al. 2013, ApJ, 766, 5

Shannon, R. M., Osłowski, S., Dai, S., et al. 2014, MNRAS, 443, 1463

Shannon, R. M., Ravi, V., Coles, W. A., et al. 2013, Sci, 342, 334

Taylor, J. H. 1992, RSPTA, 341, 117

Taylor, J. H., \& Weisberg, J. M. 1989, ApJ, 345, 434

van Haasteren, R., \& Levin, Y. 2013, MNRAS, 428, 1147

van Haasteren, R., Levin, Y., Janssen, G. H., et al. 2011, MNRAS, 414, 3117

van Haasteren, R., \& Vallisneri, M. 2014, PhRvD, 90, 104012

van Haasteren, R., \& Vallisneri, M. 2015, MNRAS, 446, 1170

van Straten, W., Demorest, P., \& Osłowski, S. 2012, AR\&T, 9, 237

Vilenkin, A., \& Shellard, E. P. S. 1994, Cosmic Strings and Other Topological Defects (Cambridge: Cambridge Univ. Press)

Wang, J. B., Hobbs, G., Coles, W., et al. 2015, MNRAS, 446, 1657

Weisberg, J. M., Nice, D. J., \& Taylor, J. H. 2010, ApJ, 722, 1030

Zhu, W. W., Stairs, I. H., Demorest, P. B., et al. 2015, ApJ, 809, 41 